\title{
Synthesis of $\alpha$-quaternary bicyclo[1.1.1]pentanes through synergistic organophotoredox and hydrogen atom transfer catalysis
}

Jeremy Nugent, $\uparrow$ Alistair J. Sterling, $\uparrow$ Nils Frank, $\uparrow$ James J. Mousseau $\downarrow$ and Edward A. Anderson*†

Corresponding author: edward.anderson@chem.ox.ac.uk

$†$ Chemistry Research Laboratory, University of Oxford, 12 Mansfield Road, Oxford OX1 3TA, U.K.

\$ Pfizer Worldwide Research and Development, Eastern Point Road, Groton, Connecticut 06340, United States

\section{Supporting Information}




\section{Contents}

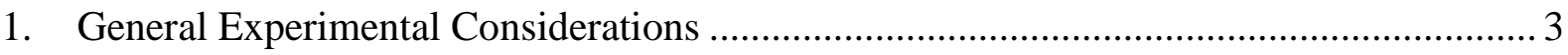

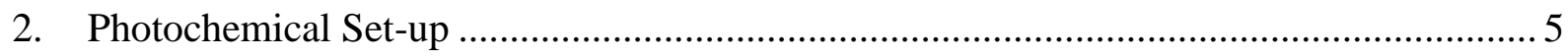

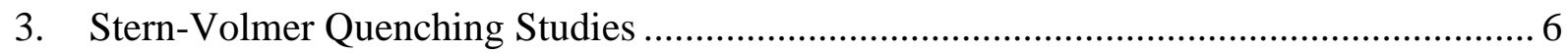

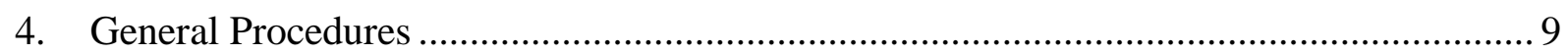

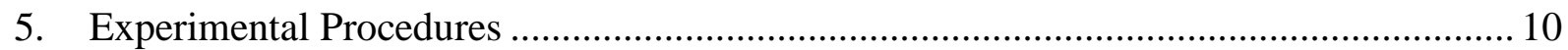

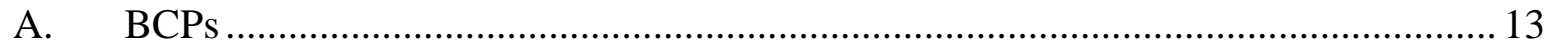

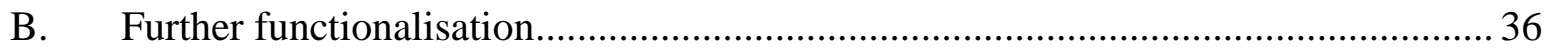

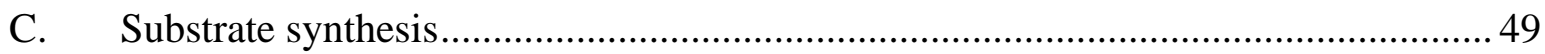

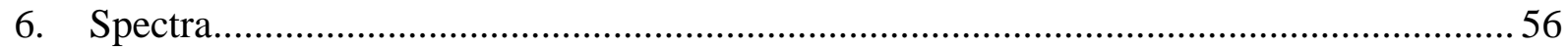

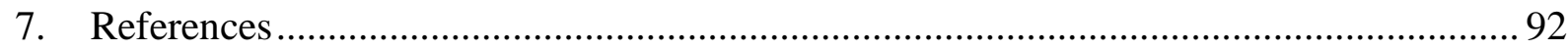




\section{General Experimental Considerations}

NMR Spectroscopy: ${ }^{1} \mathrm{H}$, and ${ }^{13} \mathrm{C}$ spectra were recorded on Bruker AV400 spectrometers using TOPSPIN software, with the deuterated solvent acting as the internal deuterium lock. ${ }^{1} \mathrm{H}$ NMR spectra were recorded at $400 \mathrm{MHz},{ }^{13} \mathrm{C} \mathrm{NMR}$ spectra were recorded at $101 \mathrm{MHz}$ with ${ }^{1} \mathrm{H}$ decoupling. Peak multiplicities are defined as: $\mathrm{s}=$ singlet, $\mathrm{d}=$ doublet, $\mathrm{t}=$ triplet, $\mathrm{q}=$ quartet, quin. $=$ quintet, $\mathrm{m}=$ multiplet, br. $=$ broad, app. $=$ apparent; coupling constants $(J)$ are reported to the nearest $0.1 \mathrm{~Hz}$.

Infrared Spectroscopy: Infrared spectra were recorded on a Bruker Tensor 27 FT-IR spectrometer with the sample being prepared as a thin film on a diamond ATR module. Absorption maxima $\left(v_{\max }\right)$ are quoted in wavenumbers $\left(\mathrm{cm}^{-1}\right)$.

High Resolution Mass Spectrometry: Accurate mass (HRMS) data was determined under conditions of ESI, EI and CI on a Bruker MicroTOF. High resolution values are calculated to 4 decimal places from the molecular formula, and all values are within a tolerance of 5 ppm.

Melting Points: Melting points were obtained using a Griffin melting point apparatus and are uncorrected.

Reagents, solvents and techniques: All air- or moisture-sensitive reactions were carried out with anhydrous solvents in flame-dried glassware under an inert atmosphere of argon. Light sensitive reactions were carried out under aluminium foil protection. Heating was performed using a sand bath. Anhydrous tetrahydrofuran (THF), dichloromethane $\left(\mathrm{CH}_{2} \mathrm{Cl}_{2}\right)$, diethyl ether $\left(\mathrm{Et}_{2} \mathrm{O}\right)$, dimethoxyethane (DME), triethylamine $\left(\mathrm{Et}_{3} \mathrm{~N}\right)$, chloroform $\left(\mathrm{CHCl}_{3}\right)$, pyridine, toluene $(\mathrm{PhMe}), \mathrm{N}, \mathrm{N}$-dimethylformamide (DMF), acetonitrile $(\mathrm{MeCN})$ and 1,4-dioxane were collected fresh from an mBraun SPS-800 solvent purification system, having been passed through anhydrous alumina columns. All other commercially available reagents and solvents were used directly as supplied. 1,3-dicarbonyl starting materials were either purchased from commercial suppliers or provided by Pfizer.

Chromatography: Column chromatography refers to normal phase column chromatography and was performed on silica gel obtained from Merck (Silica gel Si 60, 0.040-0.063 mm) under a positive pressure of nitrogen, using the stated solvent system. Analytical thin-layer chromatography was used to monitor reaction progress and performed on pre-coated aluminium-backed plates (Merck Kieselgel 60 F254 plates) with visualization by ultraviolet light $(254 \mathrm{~nm})$ and/or by staining with vanillin, phosphomolybdic acid, potassium permanganate or ninhydrin. Retention factors $\left(\mathrm{R}_{f}\right)$ are reported with the solvent system in parentheses. High performance liquid chromatography (HPLC) for enantiomeric excess (ee) determination was carried out using a DAICEL CHIRALPAK IB or IC (250 mm x $4.6 \mathrm{~mm}$ ) column (wavelength: 210-250 nm) with purified material. 
Polarimetry: Optical rotations were recorded on a Perkin Elmer 241 or 341 polarimeter with a path length of $1 \mathrm{dm}$ (using the sodium D line, $589 \mathrm{~nm}$ ). [ $\alpha]_{\mathrm{D}}$ are reported in units of $10^{-1} \mathrm{deg}$ $\mathrm{cm}^{2} \mathrm{~g}^{-1}$. Concentrations are reported in $\mathrm{g} / 100 \mathrm{~mL}$. Temperatures are reported in ${ }^{\circ} \mathrm{C}$. 


\section{Photochemical Set-up}

Under optimised conditions, reactions on $0.2 \mathrm{mmol}$ scale were irradiated with a Kessil PR160L $440 \mathrm{~nm}$ LED lamp in an Evoluchem ${ }^{\mathrm{TM}}$ PhotoRedOx box (left).

Reactions performed on $\geq 2$ mmol scale were irradiated directly with a Kessil PR160L $440 \mathrm{~nm}$ LED lamp from a distance of $5 \mathrm{~cm}$ with fan cooling (right).

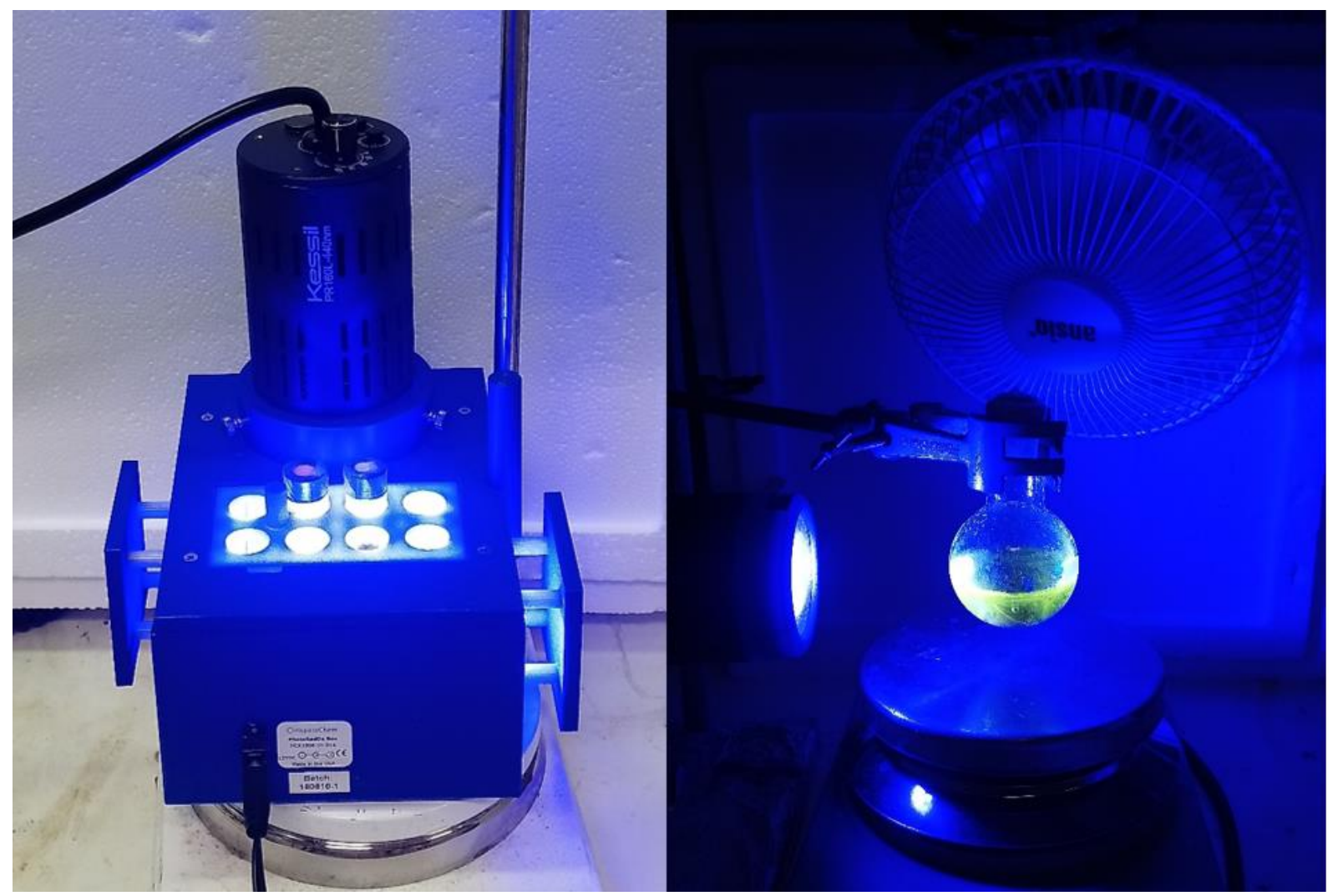

Figure S1. Photochemical set-up 


\section{Stern-Volmer Quenching Studies}

Emission spectra were recorded at $20{ }^{\circ} \mathrm{C}$ using an Edinburgh Instruments FS5 spectrofluorometer, equipped with a xenon arc lamp (390 nm excitation), an SC-20 thermostatic sample holder, and a Hamamatsu R13456 PMT detector measuring at $450-750 \mathrm{~nm}$. A quartz cuvette (10 mm path length) was charged with $2.5 \mathrm{~mL}$ of a $5 \mu \mathrm{M}$ solution of $4 \mathrm{CzIPN}$ in MeCN and was degassed by sparging with argon for 10 minutes. The appropriate volume of a $0.5 \mathrm{M}$ solution (in $\mathrm{MeCN}$ ) of ketoester $\mathbf{2 k}$ or [1.1.1]propellane (1) was added sequentially and the emission of the solution was measured (Figure S2 and S3). Each curve was integrated to obtain the fluorescence intensity (I) for a given concentration.

Stern-Volmer fluorescence quenching plots for ketoester 2k (Figure S4) and [1.1.1]propellane (1) (Figure S5) were obtained from the measured intensities using Equation (1), after adjusting for the increasing dilution of the photocatalyst.

$$
\frac{I_{0}}{I}=1+k_{q} \tau_{0}[Q]
$$

The measured lifetime of the excited state of $4 \mathrm{CzIPN}$ in MeCN in the absence of a quencher is $12.7 \mathrm{~ns},{ }^{1}$ leading to a quenching rate constant of $k_{\mathrm{q}}$ of $6 \times 10^{7} \mathrm{M}^{-1} \mathrm{~s}^{-1}$ with ketoester $2 \mathbf{k}$. The negligible quenching by [1.1.1]propellane (1), in combination with a possible chemical reaction between propellane and the photocatalyst, meant no sensible quenching rate constant could be obtained.

As a result, we can conclude that ketoester $\mathbf{2} \mathbf{k}$ is the most likely quencher of the photocatalyst under the reaction conditions. 


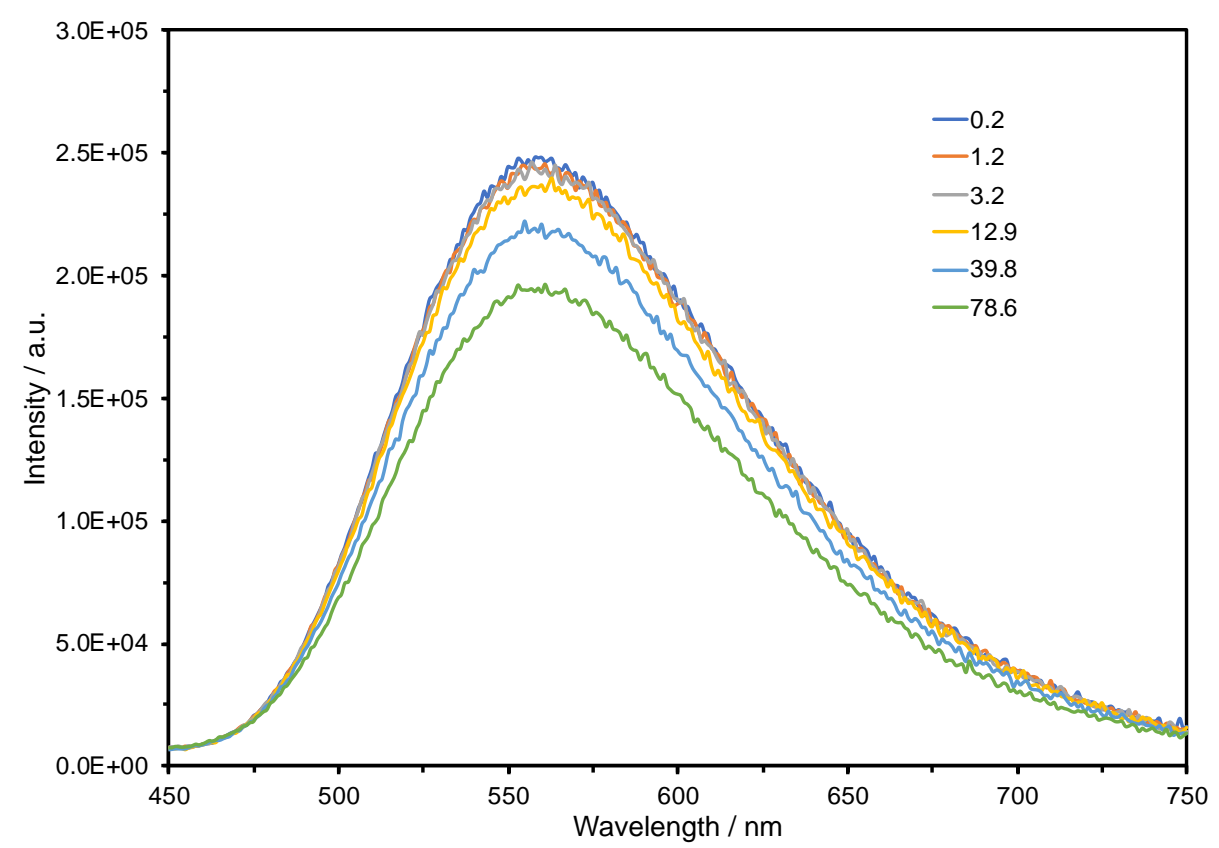

Figure S2. Fluorescence quenching titration for ketoester $\mathbf{2 k}$ with a $5 \mu \mathrm{M}$ solution of $4 \mathrm{CzIPN}$.

Quencher concentrations reported in $\mathrm{mM}$.

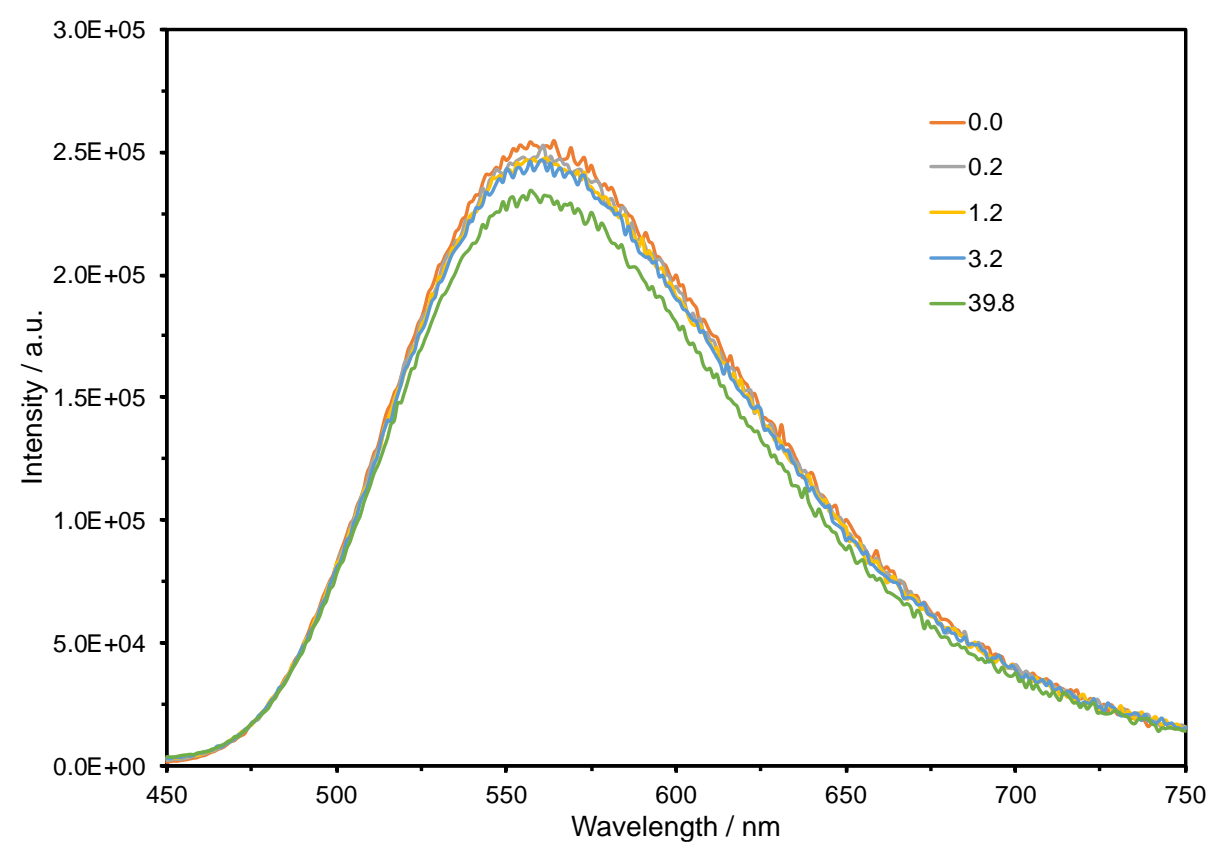

Figure S3. Fluorescence quenching titration for [1.1.1]propellane (1) with a $5 \mu \mathrm{M}$ solution of 4CzIPN. Quencher concentrations reported in mM. 


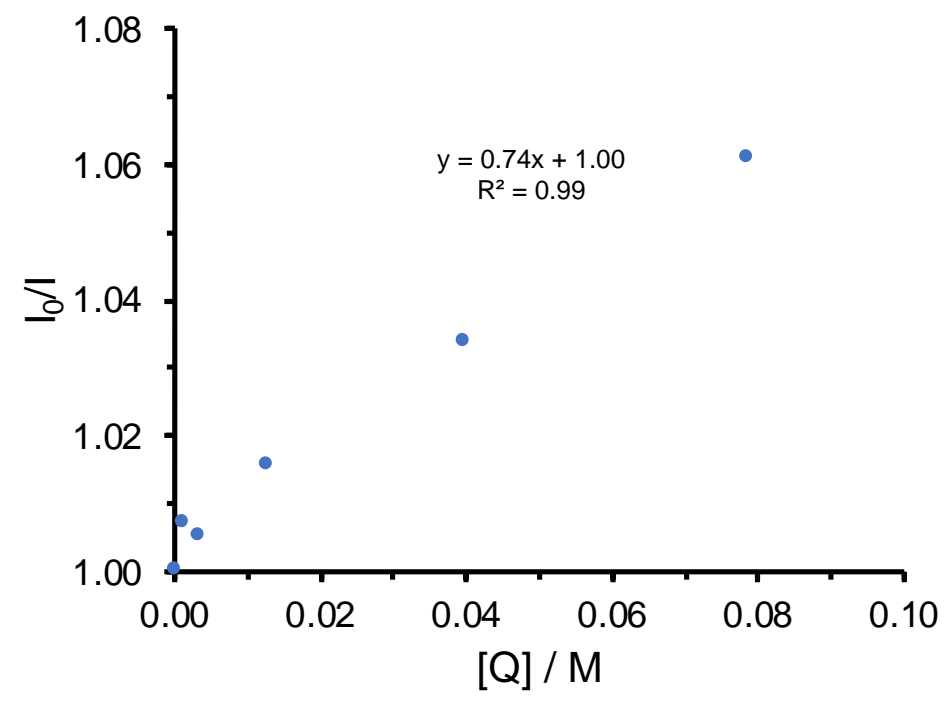

Figure S4. Stern-Volmer fluorescence quenching plot for ketoester $\mathbf{2 k}$ with a $5 \mu \mathrm{M}$ solution of $4 \mathrm{CzIPN}$.

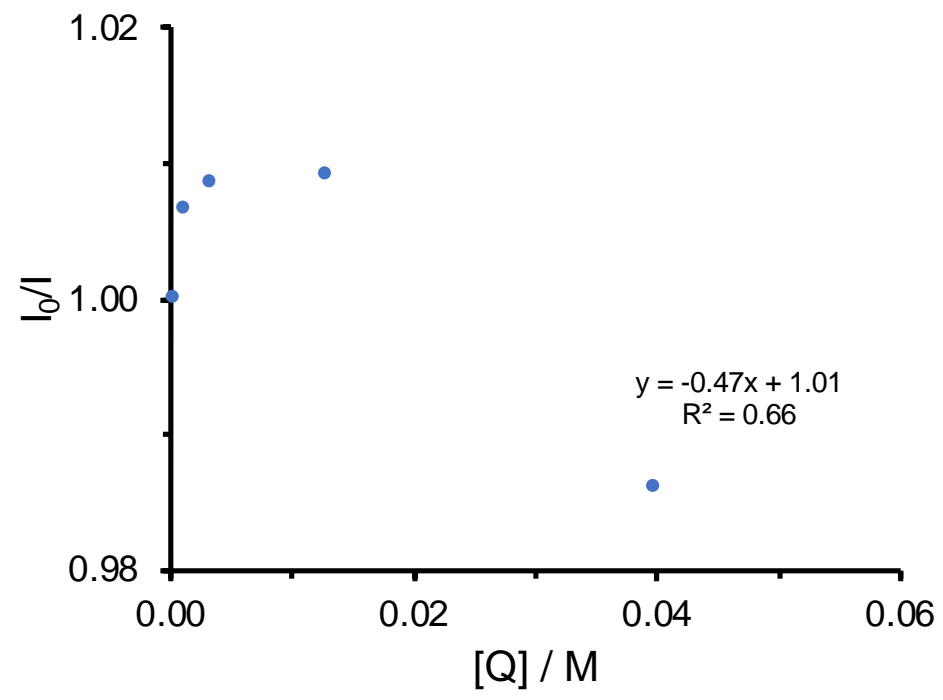

Figure S5. Stern-Volmer fluorescence quenching plot for [1.1.1]propellane (1) with a $5 \mu \mathrm{M}$ solution of $4 \mathrm{CzIPN}$. 


\section{General Procedures}

\section{General Procedure 1: 1,3-dicarbonyl bicyclopentylation}

To a flame-dried vial containing $\beta$-substituted ketones ( $0.30 \mathrm{mmol}, 1.5$ eq.), H3 (5.7 mg, 0.02 mmol, 0.1 eq) and 4CzIPN (3.9 mg, $2.5 \mathrm{~mol} \%$ ) was added anhydrous MeCN (2 mL). The vial was cooled in an ice bath and sparged with argon for $10 \mathrm{~min}$, then [1.1.1]propellane $(0.28 \mathrm{~mL}$, $0.2 \mathrm{mmol}, 1.0$ eq.) was added. The vial was sealed and irradiated with a $440 \mathrm{~nm}$ Kessil PR160L LED lamp for $20 \mathrm{~h}$ then concentrated and purified by column chromatography. 


\section{Experimental Procedures}

\section{[1.1.1]propellane (TCP)}

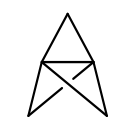

To a flame-dried round-bottom flask equipped with a stirrer bar was added 1,1-dibromo-2,2bis(chloromethyl)cyclopropane $(5.0 \mathrm{~g}, 16.9 \mathrm{mmol}, 1.0$ equiv.). The reaction vessel was evacuated and back-filled with argon three times, and then anhydrous $\mathrm{Et}_{2} \mathrm{O}(10 \mathrm{~mL})$ was added. The reaction vessel was cooled to $-45^{\circ} \mathrm{C}$ (dry ice / isopropanol bath). Phenyllithium (17.8 mL, 1.9 $\mathrm{M}$ in $\mathrm{Bu}_{2} \mathrm{O}, 33.7 \mathrm{mmol}, 2.0$ equiv.) was added dropwise over $15 \mathrm{~min}$ at $-45^{\circ} \mathrm{C}$, and the resulting mixture was stirred for $15 \mathrm{~min}$ at $-45^{\circ} \mathrm{C}$. The cooling bath was replaced with an ice bath, and the reaction mixture was warmed to $0{ }^{\circ} \mathrm{C}$, and then stirred at this temperature for $2 \mathrm{~h}$. The mixture was then distilled at room temperature (10 mbar) using a rotary evaporator, the receiving flask of which was immersed in a dry ice / acetone bath. The TCP-containing distillate (12 mL, TCP concentration $0.8 \mathrm{M}$ in $\mathrm{Et}_{2} \mathrm{O}, 54 \%$ ) was transferred to a flame-dried septum-sealed bottle under an inert atmosphere, and stored at $-20{ }^{\circ} \mathrm{C}$. The yield was determined by ${ }^{1} \mathrm{H}$ NMR spectroscopy with 1,2-dichloroethane as an internal standard. 
2-Bromo-1,3,5-tri-tert-butylbenzene<smiles>CC(C)(C)c1cc(C(C)(C)C)c(Br)c(C(C)(C)C)c1</smiles>

Prepared according to a literature procedure. ${ }^{2}$

A solution of 2,4,6-tri-tert-butylbenzene $(9.9 \mathrm{~g}, 40 \mathrm{mmol})$ in trimethyl phosphate $(100 \mathrm{~mL})$ was heated at $50{ }^{\circ} \mathrm{C}$ then added $\mathrm{Br}_{2}$ dropwise $(4.6 \mathrm{~mL}, 90 \mathrm{mmol})$. The resulting red solution was heated at $100{ }^{\circ} \mathrm{C}$ for $22 \mathrm{~h}$ then cooled to $\mathrm{RT}$ and the precipitated yellow product was filtered and washed with water $(20 \mathrm{~mL})$. The resulting solid was triturated with EtOH $(20 \mathrm{~mL})$ to afford 2-bromo-1,3,5-tri-tert-butylbenzene $(9.8 \mathrm{~g}, 75 \%)$ as a white solid.

${ }^{1} \mathbf{H}$ NMR $\left(400 \mathrm{MHz}, \mathrm{CDCl}_{3}\right) \delta 7.41$ (s, 2H), 1.59 (s, 18H), $1.32(\mathrm{~s}, 9 \mathrm{H})$.

Analytical data matches that previously reported. ${ }^{2}$ 


\section{2,4,6-tri-tert-butylbenzenethiol, $\mathrm{H3}$}<smiles>CC(C)(C)c1cc(C(C)(C)C)c(S)c(C(C)(C)C)c1</smiles>

\section{Prepared according to a literature procedure. ${ }^{3}$}

To a flame-dried 2-necked flask was added Mg turnings (389 mg, $8.0 \mathrm{mmol}$ ), anhydrous THF $(15 \mathrm{~mL})$ and DIBAL-H $(0.3 \mathrm{~mL}, 1.0 \mathrm{M}$ in hexanes), and the resulting mixture was stirred at RT for 5 mins. 2-Bromo-1,3,5-tri-tert-butylbenzene (2.6 g, $8.0 \mathrm{mmol})$ was added in one portion and the reaction was heated at reflux for $24 \mathrm{~h}$. The reaction was cooled to RT then sulfur (2.7 $\mathrm{g}, 8.4 \mathrm{mmol}$ ) was added and the resulting mixture heated at reflux for $1.5 \mathrm{~h}$. The reaction was cooled to RT then $\mathrm{LiAlH}_{4}\left(1.0 \mathrm{~mL}, 4.0 \mathrm{mmol}, 4.0 \mathrm{M}\right.$ in $\left.\mathrm{Et}_{2} \mathrm{O}\right)$ was added slowly (Caution: effervescence). After 30 mins, the reaction was cooled to $0{ }^{\circ} \mathrm{C}$ and quenched slowly with water until effervescence stopped. $1 \mathrm{M} \mathrm{HCl}(15 \mathrm{~mL})$ and $\mathrm{Et}_{2} \mathrm{O}(15 \mathrm{~mL})$ were added, and the layers separated. The aqueous layer was extracted with $\mathrm{Et}_{2} \mathrm{O}(3 \times 20 \mathrm{~mL})$ and the combined organic layers were washed with brine, dried $\left(\mathrm{MgSO}_{4}\right)$, filtered and concentrated in vacuo. The residue was recrystallised from hot EtOH to give $\mathbf{H 3}$ (900 mg, 40\%) as a white powder.

${ }^{1} \mathbf{H}$ NMR $\left(400 \mathrm{MHz}, \mathrm{C}_{6} \mathrm{D}_{6}\right) \delta 7.56(\mathrm{~s}, 2 \mathrm{H}), 3.43(\mathrm{~s}, 1 \mathrm{H}), 1.61(\mathrm{~s}, 18 \mathrm{H}), 1.30(\mathrm{~s}, 9 \mathrm{H})$.

Analytical data matches that previously reported. ${ }^{3}$

Note: The mother liquor can be concentrated, and the residue recrystallised from hot EtOH to afford a second crop of product which is usually contaminated with 20-30\% 1,3,5-tri-tertbutylbenzene. We found that this impure $\mathbf{H 3}$ could be used in General procedure 1 without issue. 


\section{A. BCPs}

\section{3-Acetyl-3-(bicyclo[1.1.1]pentan-1-yl)dihydrofuran-2(3H)-one, 3a}

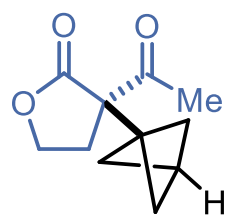

$\alpha$-Acetylbutyrolactone (32 $\mu \mathrm{L}, 0.30 \mathrm{mmol}, 1.5$ eq.) was submitted to General Procedure 1 . Purification by column chromatography ( $\mathrm{SiO}_{2}$, pentane / EtOAc, 4:1) gave 3a (36 mg, 93\%) as a pale-yellow oil.

$\mathbf{R}_{f} 0.33$ (pentane / EtOAc, 4:1);

${ }^{1} \mathbf{H}$ NMR $\left(400 \mathrm{MHz}, \mathrm{CDCl}_{3}\right) \delta 4.30-4.02(\mathrm{~m}, 2 \mathrm{H}), 2.78(\mathrm{ddd}, J=13.4,7.9,5.8 \mathrm{~Hz}, 1 \mathrm{H}), 2.59$ (s, 1H), $2.36(\mathrm{~s}, 3 \mathrm{H}), 2.20$ (ddd, $J=13.4,8.2,6.8 \mathrm{~Hz}, 1 \mathrm{H}), 1.92-1.80(\mathrm{~m}, 6 \mathrm{H})$;

${ }^{13} \mathrm{C}$ NMR (101 MHz, $\left.\mathrm{CDCl}_{3}\right) \delta 201.5,174.0,65.6,62.3,49.5,45.1,28.3,27.9,27.0$;

HRMS (ESI) m/z: [M+H] $]^{+}$calcd for $\mathrm{C}_{11} \mathrm{H}_{15} \mathrm{O}_{3}$ 195.1016, found 195.1018;

IR (film, $v_{\max } / \mathrm{cm}^{-1}$ ) 2973, 2914, 2877, 1761, 1709, 1140, 1027. 
Methyl 1-(bicyclo[1.1.1]pentan-1-yl)-2-oxocyclopentane-1-carboxylate, 3b

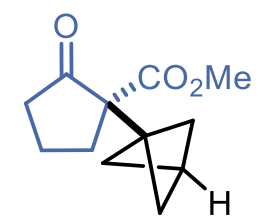

Methyl 2-oxocyclopentanecarboxylate ( $37 \mu \mathrm{L}, 0.30 \mathrm{mmol}, 1.5$ eq.) was submitted to General Procedure 1. Purification by column chromatography $\left(\mathrm{SiO}_{2}\right.$, pentane / EtOAc, 4:1) gave $3 \mathbf{b}$ (36 $\mathrm{mg}, 86 \%$ ) as a pale-yellow oil.

$\mathbf{R}_{f} 0.33$ (pentane / EtOAc, 9:1);

${ }^{1} \mathbf{H}$ NMR $\left(400 \mathrm{MHz}, \mathrm{CDCl}_{3}\right) \delta 3.69$ (s, 3H), 2.50 (s, 1H), $2.44-2.29$ (m, 2H), 2.22 (ddd, $J=$ $18.7,8.6,6.9 \mathrm{~Hz}, 1 \mathrm{H}), 2.06(\mathrm{dt}, J=13.6,6.9 \mathrm{~Hz}, 1 \mathrm{H}), 1.98-1.76(\mathrm{~m}, 8 \mathrm{H})$;

${ }^{13} \mathbf{C} \mathbf{N M R}\left(101 \mathrm{MHz}, \mathrm{CDCl}_{3}\right) \delta 212.9,170.7,60.8,52.3,49.5,45.3,38.8,32.0,27.9,19.8 ;$

HRMS (ESI) m/z: [M+H] $]^{+}$calcd for $\mathrm{C}_{12} \mathrm{H}_{17} \mathrm{O}_{3}$ 209.1172, found 209.1173;

IR (film, $v_{\max } / \mathrm{cm}^{-1}$ ) 2970, 2912, 2876, 1751, 1727, 1198. 
Methyl 3-(bicyclo[1.1.1]pentan-1-yl)-4-oxo-1-phenylpyrrolidine-3-carboxylate, 3c<smiles>CO[C@]12C(=O)CN(c3ccccc3)[C@@H]1C1CC2C2CC21</smiles>

Methyl 4-oxo-1-phenylpyrrolidine-3-carboxylate (66 mg, $0.30 \mathrm{mmol}, 1.5$ eq.) was submitted to General Procedure 1. Purification by column chromatography ( $\mathrm{SiO}_{2}$, pentane / EtOAc, 9:1) gave 3c (53 mg, 93\%) as a pale-yellow solid.

m.p. $109-111^{\circ} \mathrm{C}\left(\mathrm{CH}_{2} \mathrm{Cl}_{2}\right)$

$\mathbf{R}_{f} 0.42$ (pentane / EtOAc, 9:1);

${ }^{1} \mathbf{H}$ NMR $\left(400 \mathrm{MHz}, \mathrm{CDCl}_{3}\right) \delta 7.40-7.28(\mathrm{~m}, 2 \mathrm{H}), 6.85(\mathrm{t}, J=7.3 \mathrm{~Hz}, 1 \mathrm{H}), 6.68(\mathrm{~d}, J=8.0$ $\mathrm{Hz}, 2 \mathrm{H}), 4.08(\mathrm{~d}, J=9.9 \mathrm{~Hz}, 1 \mathrm{H}), 3.85(\mathrm{~d}, J=18.4 \mathrm{~Hz}, 1 \mathrm{H}), 3.77$ (s, 3H), 3.72 (d, $J=18.4 \mathrm{~Hz}$, $1 \mathrm{H}), 3.66(\mathrm{~d}, J=9.9 \mathrm{~Hz}, 1 \mathrm{H}), 2.56(\mathrm{~s}, 1 \mathrm{H}), 1.94-1.84(\mathrm{~m}, 7 \mathrm{H})$;

${ }^{13} \mathbf{C}$ NMR(101 MHz, $\left.\mathrm{CDCl}_{3}\right) \delta 205.4,168.6,147.2,129.6,118.6,112.9,61.6,56.0,52.9,52.7$, 49.5, 44.4, 27.7;

HRMS (ESI) m/z: $[\mathrm{M}+\mathrm{H}]^{+}$calcd for $\mathrm{C}_{17} \mathrm{H}_{20} \mathrm{NO}_{3} 286.1438$, found 286.1437;

IR (film, $\left.v_{\max } / \mathrm{cm}^{-1}\right)$ 2989, 2970, 2916, 2879, 1731, 1727. 
3-(bicyclo[1.1.1]pentan-1-yl)-3-isonicotinoyldihydrofuran-2(3H)-one, 3d<smiles>O=C(c1ccncc1)C12C[C@@H]1C1COC(=O)[C@]12C1CC1</smiles>

3-Isonicotinoyldihydrofuran-2(3H)-one ( $57 \mathrm{mg}, 0.30 \mathrm{mmol}, 1.5 \mathrm{eq}$.) was submitted to General Procedure 1. Purification by column chromatography ( $\mathrm{SiO}_{2}$, EtOAc, 9:1) gave 3d (45 mg, 88\%) as a pale-yellow solid.

m.p. $54-56{ }^{\circ} \mathrm{C}\left(\mathrm{CH}_{2} \mathrm{Cl}_{2}\right)$;

$\mathbf{R}_{f} 0.31$ (EtOAc);

${ }^{1} \mathbf{H}$ NMR (400 MHz, $\left.\mathrm{CDCl}_{3}\right) 8.79$ - $8.73(\mathrm{~m}, 2 \mathrm{H}), 7.78$ - $7.72(\mathrm{~m}, 2 \mathrm{H}), 4.43-4.14(\mathrm{~m}, 2 \mathrm{H})$, $2.93(\mathrm{ddd}, J=13.2,7.0,6.1 \mathrm{~Hz}, 1 \mathrm{H}), 2.58(\mathrm{~s}, 1 \mathrm{H}), 2.43(\mathrm{dt}, J=13.2,7.5 \mathrm{~Hz}, 1 \mathrm{H}), 1.98-1.79$ $(\mathrm{m}, 6 \mathrm{H})$;

${ }^{13}$ C NMR (101 MHz, $\left.\mathrm{CDCl}_{3}\right) \delta$ 194.5, 173.6, 150.7, 143.0, 122.2, 66.1, 60.9, 49.7, 45.1, 31.1, 28.0

HRMS (ESI) m/z: $[\mathrm{M}+\mathrm{H}]^{+}$calcd for $\mathrm{C}_{15} \mathrm{H}_{16} \mathrm{NO}_{3}$ 258.1125, found 258.1125;

IR (film, $v_{\max } / \mathrm{cm}^{-1}$ ) 2979, 2915, 2880, 1777, 1764. 
Ethyl 1-benzyl-3-(bicyclo[1.1.1]pentan-1-yl)-4,5-dioxopyrrolidine-3-carboxylate, 3e

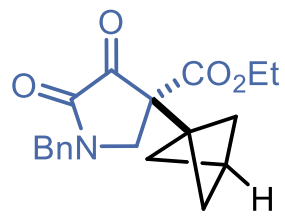

Ethyl 1-benzyl-4,5-dioxopyrrolidine-3-carboxylate (78 mg, $0.30 \mathrm{mmol}, 1.5$ eq.) was submitted to General Procedure 1. Purification by column chromatography $\left(\mathrm{SiO}_{2}\right.$, pentane / EtOAc, $\left.4: 1\right)$ gave $\mathbf{3 e}$ (33 $\mathrm{mg}, 50 \%)$ as a pale-yellow solid.

m.p. $84-86{ }^{\circ} \mathrm{C}\left(\mathrm{CH}_{2} \mathrm{Cl}_{2}\right)$

$\mathbf{R}_{f} 0.43$ (pentane / EtOAc, 1:1);

${ }^{1}$ H NMR $\left(400 \mathrm{MHz}, \mathrm{CDCl}_{3}\right) \delta 7.49-7.27(\mathrm{~m}, 5 \mathrm{H}), 4.82(\mathrm{~d}, J=14.4 \mathrm{~Hz}, 1 \mathrm{H}), 4.57$ (d, $J=14.4$ $\mathrm{Hz}, 1 \mathrm{H}), 4.26-4.07(\mathrm{~m}, 2 \mathrm{H}), 3.88(\mathrm{~d}, J=11.1 \mathrm{~Hz}, 1 \mathrm{H}), 3.41(\mathrm{~d}, J=11.1 \mathrm{~Hz}, 1 \mathrm{H}), 2.50(\mathrm{~s}, 1 \mathrm{H})$, $1.78-1.66(\mathrm{~m}, 6 \mathrm{H}), 1.23(\mathrm{t}, J=7.1 \mathrm{~Hz}, 3 \mathrm{H})$;

${ }^{13} \mathbf{C}$ NMR(101 MHz, $\left.\mathrm{CDCl}_{3}\right) \delta 192.7,166.5,157.8,134.3,129.2,128.8,128.7,62.4,55.6,49.1$, 48.7, 47.4, 44.7, 28.1, 14.2;

HRMS (ESI) m/z: $[\mathrm{M}+\mathrm{H}]^{+}$calcd for $\mathrm{C}_{19} \mathrm{H}_{22} \mathrm{NO}_{4}$ 328.1543, found 328.1544;

IR (film, $v_{\max } / \mathrm{cm}^{-1}$ ) 2980, 2971, 2916, 1775, 1732, 1710. 
carboxylate, $3 f$

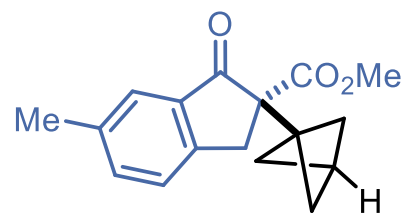

Methyl 6-methyl-1-oxo-2,3-dihydro- $1 H$-indene-2-carboxylate (61 mg, $0.30 \mathrm{mmol}, 1.5$ eq.) was submitted to General Procedure 1. Purification by column chromatography $\left(\mathrm{SiO}_{2}\right.$, pentane / EtOAc, 95:5) gave $3 f$ (49 mg, 91\%) as a white solid.

m.p. $89-91{ }^{\circ} \mathrm{C}\left(\mathrm{CH}_{2} \mathrm{Cl}_{2}\right)$

$\mathbf{R}_{f} 0.49$ (pentane / EtOAc, 4:1);

${ }^{1} \mathbf{H}$ NMR $\left(400 \mathrm{MHz}, \mathrm{CDCl}_{3}\right) \delta 7.54(\mathrm{~s}, 1 \mathrm{H}), 7.41(\mathrm{dd}, J=7.8,1.0 \mathrm{~Hz}, 1 \mathrm{H}), 7.34(\mathrm{~d}, J=7.8 \mathrm{~Hz}$, 1H), 3.70 (s, 3H), 3.53 (d, $J=16.9 \mathrm{~Hz}, 1 \mathrm{H}), 3.16$ (d, $J=16.9 \mathrm{~Hz}, 1 \mathrm{H}), 2.49$ (s, 1H), 2.39 (s, $3 \mathrm{H}), 1.88-1.72(\mathrm{~m}, 6 \mathrm{H})$;

${ }^{13}$ C NMR (101 MHz, $\left.\mathrm{CDCl}_{3}\right) \delta$ 200.2, 170.7, 150.0, 137.8, 136.6, 135.7, 126.0, 124.6, 61.5, 52.4, 49.1, 45.8, 35.0, 27.6, 21.2;

HRMS (ESI) m/z: [M+H] $]^{+}$calcd for $\mathrm{C}_{17} \mathrm{H}_{19} \mathrm{O}_{3}$ 271.1329, found 271.1329;

IR (film, $v_{\max } / \mathrm{cm}^{-1}$ ) 2997, 2954, 2917, 2874, 1737, 1709. 
carboxylate, $3 g$

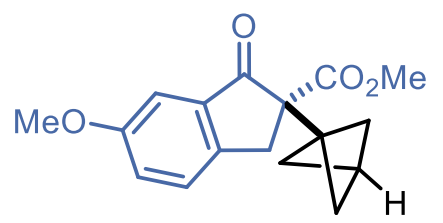

$2 \mathrm{~g}$ (66 mg, $0.30 \mathrm{mmol}, 1.5$ eq.) was submitted to General Procedure 1. Purification by column chromatography $\left(\mathrm{SiO}_{2}\right.$, pentane / EtOAc, 95:5) gave $\mathbf{3 g}(51 \mathrm{mg}, 89 \%)$ as a white solid.

m.p. $118-119{ }^{\circ} \mathrm{C}\left(\mathrm{CH}_{2} \mathrm{Cl}_{2}\right)$

$\mathbf{R}_{f} 0.34$ (pentane / EtOAc, 4:1);

${ }^{1} \mathbf{H}$ NMR $\left(400 \mathrm{MHz}, \mathrm{CDCl}_{3}\right) \delta 7.33(\mathrm{~d}, J=8.2 \mathrm{~Hz}, 1 \mathrm{H}), 7.23-7.14(\mathrm{~m}, 2 \mathrm{H}), 3.82(\mathrm{~s}, 3 \mathrm{H}), 3.70$ (s, 3H), $3.48(\mathrm{~d}, J=16.9 \mathrm{~Hz}, 1 \mathrm{H}), 3.13(\mathrm{~d}, J=16.9 \mathrm{~Hz}, 1 \mathrm{H}), 2.49(\mathrm{~s}, 1 \mathrm{H}), 1.87-1.70(\mathrm{~m}, 6 \mathrm{H})$;

${ }^{13} \mathrm{C}$ NMR (101 MHz, $\left.\mathrm{CDCl}_{3}\right) \delta$ 200.1, 170.6, 159.8, 145.5, 136.7, 127.1, 124.8, 105.7, 62.0, 55.7, 52.4, 49.1, 45.8, 34.7, 27.6;

HRMS (ESI) m/z: [M+H] $]^{+}$calcd for $\mathrm{C}_{17} \mathrm{H}_{19} \mathrm{O}_{4}$ 287.1278, found 287.1280;

IR (film, $v_{\max } / \mathrm{cm}^{-1}$ ) 2984, 2957, 1705, 1492, 1439. 
Dimethyl 3-(bicyclo[1.1.1]pentan-1-yl)-4-oxopiperidine-1,3-dicarboxylate, $3 \mathrm{~h}$<smiles>COC(=O)N1CCC(=O)[C@]2(C)C3CC([C@H]4C[C@H]34)[C@]12C</smiles>

2h (65 mg, $0.30 \mathrm{mmol}, 1.5$ eq.) was submitted to General Procedure 1. Purification by column chromatography ( $\mathrm{SiO}_{2}$, pentane / EtOAc, 4:1) gave $\mathbf{3 h}$ (43 mg, 77\%) as a pale-yellow solid.

m.p. $77-79{ }^{\circ} \mathrm{C}\left(\mathrm{CH}_{2} \mathrm{Cl}_{2}\right)$

$\mathbf{R}_{f} 0.15$ (pentane / EtOAc, 4:1);

${ }^{1} \mathbf{H}$ NMR $\left(400 \mathrm{MHz}, \mathrm{CDCl}_{3}\right) \delta 4.50(\mathrm{dd}, J=13.6,1.9 \mathrm{~Hz}, 1 \mathrm{H}), 4.18-3.97(\mathrm{~m}, 1 \mathrm{H}), 3.75(\mathrm{~s}$, $3 \mathrm{H}), 3.73$ (s, 3H), $3.43-3.17(\mathrm{~m}, 2 \mathrm{H}), 2.62(\mathrm{ddd}, J=15.0,9.9,6.3 \mathrm{~Hz}, 1 \mathrm{H}), 2.52(\mathrm{~s}, 1 \mathrm{H}), 2.41$ (dt, $J=15.0,4.8 \mathrm{~Hz}, 1 \mathrm{H}), 1.88(\mathrm{~s}, 6 \mathrm{H})$;

${ }^{13} \mathrm{C}$ NMR (101 MHz, $\left.\mathrm{CDCl}_{3}\right) \delta 203.1,169.2,155.8,61.0,53.2,52.6,49.9,48.5,44.3,43.3$, 40.3, 27.9;

HRMS (ESI) m/z: $[\mathrm{M}+\mathrm{H}]^{+}$calcd for $\mathrm{C}_{14} \mathrm{H}_{20} \mathrm{NO}_{5}$ 282.1336, found 282.1334;

IR (film, $v_{\max } / \mathrm{cm}^{-1}$ ) 2981, 2917, 2879, 1741, 1702. 
Methyl 2-(bicyclo[1.1.1]pentan-1-yl)-1-oxo-1,2,3,4-tetrahydronaphthalene-2-carboxylate, $3 \mathbf{i}$

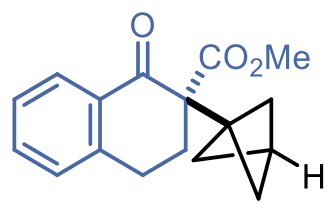

Methyl 1-oxo-1,2,3,4-tetrahydronaphthalene-2-carboxylate $(61 \mathrm{mg}, 0.30 \mathrm{mmol}, 1.5 \mathrm{eq}$.$) was$ submitted to General Procedure 1. Purification by column chromatography $\left(\mathrm{SiO}_{2}\right.$, pentane / EtOAc, 95:5) gave 3i (52 mg, 95\%) as a white solid.

m.p. $108-110{ }^{\circ} \mathrm{C}\left(\mathrm{CH}_{2} \mathrm{Cl}_{2}\right)$

$\mathbf{R}_{f} 0.18$ (pentane / EtOAc, 95:5);

${ }^{1} \mathbf{H}$ NMR $\left(400 \mathrm{MHz}, \mathrm{CDCl}_{3}\right) \delta 8.03(\mathrm{dd}, J=7.8,1.5 \mathrm{~Hz}, 1 \mathrm{H}), 7.45(\mathrm{td}, J=7.5,1.5 \mathrm{~Hz}, 1 \mathrm{H})$, $7.35-7.28(\mathrm{~m}, 1 \mathrm{H}), 7.19(\mathrm{~d}, J=7.8 \mathrm{~Hz}, 1 \mathrm{H}), 3.65$ (s, 3H), $3.08-2.97$ (m, 1H), 2.89 (ddd, $J=$ 17.3, 4.9, 3.4 Hz, 1H), $2.58-2.48$ (m, 2H), 2.08 (ddd, $J=13.6,12.0,4.9 \mathrm{~Hz}, 1 \mathrm{H}), 2.00-1.86$ $(\mathrm{m}, 6 \mathrm{H})$;

${ }^{13}$ C NMR (101 MHz, $\left.\mathrm{CDCl}_{3}\right) \delta$ 194.2, 170.5, 143.3, 133.5, 132.7, 128.8, 128.0, 126.9, 57.8, 52.4, 49.8, 47.0, 30.0, 27.3, 26.3;

HRMS (ESI) m/z: [M+H] $]^{+}$calcd for $\mathrm{C}_{17} \mathrm{H}_{19} \mathrm{O}_{3}$ 271.1329, found 271.1328;

IR (film, $v_{\max } / \mathrm{cm}^{-1}$ ) 2980, 2912, 2875, 1730, 1685. 
Ethyl 2-benzyl-2-(bicyclo[1.1.1]pentan-1-yl)-3-oxobutanoate, 3j

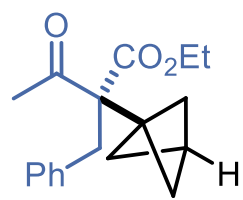

Ethyl 2-benzyl-3-oxobutanoate (66 $\mathrm{mg}, 0.30 \mathrm{mmol}, 1.5$ eq.) was submitted to General Procedure 1. Purification by column chromatography $\left(\mathrm{SiO}_{2}\right.$, pentane / EtOAc, 95:5) gave 3j (29 $\mathrm{mg}, 51 \%)$ as a pale-yellow oil.

$\mathbf{R}_{f} 0.34$ (pentane / EtOAc, 95:5);

${ }^{1} \mathbf{H}$ NMR $\left(400 \mathrm{MHz}, \mathrm{CDCl}_{3}\right) \delta 7.25$ - $7.11(\mathrm{~m}, 5 \mathrm{H}), 4.18-3.98(\mathrm{~m}, 2 \mathrm{H}), 3.31(\mathrm{~d}, J=13.7 \mathrm{~Hz}$, $1 \mathrm{H}), 2.99(\mathrm{~d}, J=13.7 \mathrm{~Hz}, 1 \mathrm{H}), 2.51(\mathrm{~s}, 1 \mathrm{H}), 2.04(\mathrm{~s}, 3 \mathrm{H}), 1.91-1.80(\mathrm{~m}, 6 \mathrm{H}), 1.15(\mathrm{t}, J=7.1$ $\mathrm{Hz}, 3 \mathrm{H})$;

${ }^{13} \mathrm{C}$ NMR(101 MHz, $\left.\mathrm{CDCl}_{3}\right) \delta 204.3,171.0,137.5,130.3,128.2,126.7,66.4,60.9,50.1,46.2$, 38.0, 30.5, 27.0, 14.1;

HRMS (ESI) m/z: [M+H] $]^{+}$calcd for $\mathrm{C}_{18} \mathrm{H}_{23} \mathrm{O}_{3}$ 287.1642, found 287.1640;

IR (film, $v_{\max } / \mathrm{cm}^{-1}$ ) 2973, 2911, 2876, 1728, 1706. 
Ethyl 2-(bicyclo[1.1.1]pentan-1-yl)-3-oxo-3-phenylpropanoate, 3k<smiles>CCOC(=O)C(C(=O)c1ccccc1)C12CCC(C1)C2</smiles>

0.2 mmol scale: Ethyl benzoylacetate ( $52 \mu \mathrm{L}, 0.30 \mathrm{mmol}, 1.5 \mathrm{eq}$.) was submitted to General Procedure 1. Purification by column chromatography $\left(\mathrm{SiO}_{2}, 50: 45: 5, \mathrm{CH}_{2} \mathrm{Cl}_{2} /\right.$ pentane / $\left.\mathrm{Et}_{2} \mathrm{O}\right)$ gave 3k (39 mg, 76\%) as a colourless oil.

2 mmol scale: To a flame-dried RB flask containing ethyl benzoylacetate $(520 \mu \mathrm{L}, 3.0 \mathrm{mmol}$, 1.5 eq.), $\mathbf{H 3}$ (56 mg, $0.20 \mathrm{mmol}, 0.1 \mathrm{eq}$ ) and 4CzIPN (39 mg, $2.5 \mathrm{~mol} \%$ ) was added anhydrous MeCN (20 mL). The flask was cooled in an ice bath and sparged with argon for $15 \mathrm{~min}$, then [1.1.1]propellane (2.7 $\mathrm{mL}$ of a $0.75 \mathrm{M}$ solution in $\mathrm{Et}_{2} \mathrm{O}, 2.0 \mathrm{mmol}, 1.0$ eq.) was added. The flask was sealed and irradiated with a $440 \mathrm{~nm}$ Kessil PR160L LED lamp for $20 \mathrm{~h}$ with fan cooling then concentrated. Purification by column chromatography $\left(\mathrm{SiO}_{2}, 50: 45: 5, \mathrm{CH}_{2} \mathrm{Cl}_{2} /\right.$ pentane / $\mathrm{Et}_{2} \mathrm{O}$ ) gave 3k (420 mg, 81\%) as a colourless oil.

$\mathbf{R}_{f} 0.50$ (pentane / EtOAc, 9:1);

${ }^{1} \mathbf{H}$ NMR $\left(400 \mathrm{MHz}, \mathrm{CDCl}_{3}\right) \delta 7.96-7.88(\mathrm{~m}, 2 \mathrm{H}), 7.62-7.53(\mathrm{~m}, 1 \mathrm{H}), 7.52-7.42(\mathrm{~m}, 2 \mathrm{H})$, $4.49(\mathrm{~s}, 1 \mathrm{H}), 4.24-4.08(\mathrm{~m}, 2 \mathrm{H}), 2.51(\mathrm{~s}, 1 \mathrm{H}), 1.95-1.84(\mathrm{~m}, 6 \mathrm{H}), 1.20(\mathrm{t}, J=7.1 \mathrm{~Hz}, 3 \mathrm{H})$;

${ }^{13} \mathrm{C} \mathbf{N M R}\left(101 \mathrm{MHz}, \mathrm{CDCl}_{3}\right) \delta 194.0,168.5,136.9,133.5,128.8,128.6,61.2,55.8,51.2,42.1$, 28.7, 14.3;

HRMS (ESI) m/z: [M+H] $]^{+}$calcd for $\mathrm{C}_{16} \mathrm{H}_{19} \mathrm{O}_{3}$ 259.1329, found 259.1329;

IR (film, $v_{\max } / \mathrm{cm}^{-1}$ ) 2973, 2912, 2876, 1734, 1683. 
carboxylate, 31

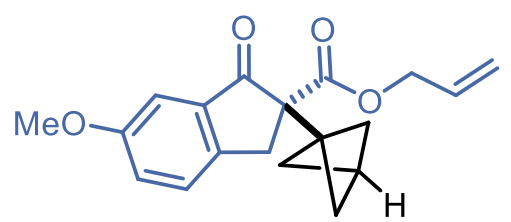

21 (74 mg, $0.30 \mathrm{mmol}, 1.5$ eq.) was submitted to General Procedure 1. Purification by column chromatography $\left(\mathrm{SiO}_{2}\right.$, pentane / EtOAc, 95:5) gave 31 (49 mg, 79\%) as a colourless oil.

$\mathbf{R}_{f} 0.21$ (pentane / EtOAc, 9:1);

${ }^{1} \mathbf{H}$ NMR $\left(400 \mathrm{MHz}, \mathrm{CDCl}_{3}\right) \delta 7.36-7.31(\mathrm{~m}, 1 \mathrm{H}), 7.22-7.17(\mathrm{~m}, 2 \mathrm{H}), 5.88(\mathrm{ddt}, J=17.2$, $10.5,5.6 \mathrm{~Hz}, 1 \mathrm{H}), 5.30(\mathrm{dq}, J=17.2,1.4 \mathrm{~Hz}, 1 \mathrm{H}), 5.21(\mathrm{dq}, J=10.5,1.4 \mathrm{~Hz}, 1 \mathrm{H}), 4.62(\mathrm{dt}, J=$ 5.6, 1.4 Hz, 2H), 3.83 (s, 3H), 3.51 (d, $J=16.9 \mathrm{~Hz}, 1 \mathrm{H}), 3.15$ (d, $J=16.9 \mathrm{~Hz}, 1 \mathrm{H}), 2.50(\mathrm{~s}, 1 \mathrm{H})$, $1.88-1.74(\mathrm{~m}, 6 \mathrm{H})$;

${ }^{13} \mathrm{C}$ NMR (101 MHz, $\left.\mathrm{CDCl}_{3}\right) \delta 200.0,169.8,159.8,145.4,136.7,132.0,127.1,124.8,118.5$, 105.8, 65.8, 62.0, 55.7, 49.1, 45.9, 34.7, 27.6;

HRMS (ESI) m/z: [M+H] $]^{+}$calcd for $\mathrm{C}_{19} \mathrm{H}_{21} \mathrm{O}_{4} 313.1434$, found 313.1434;

IR (film, $v_{\max } / \mathrm{cm}^{-1}$ ) 2970, 2912, 2874, 1743, 1708. 


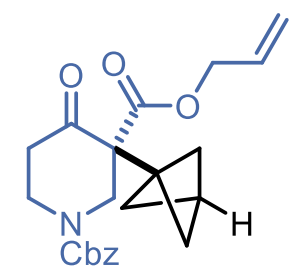

0.2 mmol scale: 3-Allyl 1-benzyl 4-oxopiperidine-1,3-dicarboxylate (2m) (95 mg, 0.30 mmol, 1.5 eq.) was submitted to General Procedure 1 . Purification by column chromatography $\left(\mathrm{SiO}_{2}\right.$, pentane / $\left.\mathrm{Et}_{2} \mathrm{O}, 7: 3\right)$ gave $\mathbf{3 m}(62 \mathrm{mg}, 81 \%)$ as a pale-yellow oil.

2 mmol scale: To a flame-dried RB flask containing $\mathbf{2 m}$ (950 mg, $3.0 \mathrm{mmol}, 1.5$ eq.), $\mathbf{H 3}$ (56 $\mathrm{mg}, 0.20 \mathrm{mmol}, 0.1 \mathrm{eq})$ and 4CzIPN (39 mg, $2.5 \mathrm{~mol} \%)$ was added anhydrous MeCN (20 mL). The flask was cooled in an ice bath and sparged with argon for $15 \mathrm{~min}$, then [1.1.1]propellane (2.7 $\mathrm{mL}$ of a $0.75 \mathrm{M}$ solution in $\mathrm{Et}_{2} \mathrm{O}, 2.0 \mathrm{mmol}, 1.0$ eq.) was added. The flask was sealed and irradiated with a $440 \mathrm{~nm}$ Kessil PR160L LED lamp for $20 \mathrm{~h}$ with fan cooling then concentrated. Purification by column chromatography $\left(\mathrm{SiO}_{2}\right.$, pentane $\left./ \mathrm{Et}_{2} \mathrm{O}, 7: 3\right)$ gave $\mathbf{3 m}(550 \mathrm{mg}, 72 \%)$ as a pale-yellow oil.

$\mathbf{R}_{f} 0.46$ (pentane / $\left.\mathrm{Et}_{2} \mathrm{O}, 1: 1\right)$;

${ }^{1} \mathbf{H}$ NMR $\left(400 \mathrm{MHz}, \mathrm{CDCl}_{3}\right) \delta 7.45$ - $7.29(\mathrm{~m}, 5 \mathrm{H}), 6.02-5.71(\mathrm{~m}, 1 \mathrm{H}), 5.40-5.04(\mathrm{~m}, 4 \mathrm{H})$, $4.66-4.51(\mathrm{~m}, 3 \mathrm{H}), 4.22-3.92(\mathrm{~m}, 1 \mathrm{H}), 3.46-3.21(\mathrm{~m}, 2 \mathrm{H}), 2.63(\mathrm{ddd}, J=15.4,9.7,6.2 \mathrm{~Hz}$, 1H), $2.50(\mathrm{~s}, 1 \mathrm{H}), 2.42(\mathrm{dt}, J=15.4,4.9 \mathrm{~Hz}, 1 \mathrm{H}), 1.88(\mathrm{~s}, 6 \mathrm{H})$;

${ }^{13}$ C NMR (101 MHz, $\left.\mathrm{CDCl}_{3}\right) \delta 203.0,168.4,155.1,136.5,131.5,128.6,128.3,128.2,119.4$, 67.7, 66.3, 61.0, 49.9, 48.5, 44.3, 43.3, 40.2, 27.9;

HRMS (ESI) m/z: $[\mathrm{M}+\mathrm{H}]^{+}$calcd for $\mathrm{C}_{22} \mathrm{H}_{26} \mathrm{O}_{5} \mathrm{~N}$ 384.1805, found 384.1807;

IR (film, $v_{\max } / \mathrm{cm}^{-1}$ ) 2973, 2913, 2877, 1742, 1701. 
<smiles>C=CCOC(=O)[C@@]12C[C@@H]3CC3C1N([13CH3])CCC2=O</smiles>

Allyl 4-oxo-1-tosylpiperidine-3-carboxylate (2n) (101 mg, $0.30 \mathrm{mmol}, 1.5$ eq.) was submitted to General Procedure 1. Purification by column chromatography $\left(\mathrm{SiO}_{2}\right.$, pentane $\left./ \mathrm{Et}_{2} \mathrm{O}, 7: 3\right)$ gave 3n (57 mg, 68\%) as a yellow oil. Trace 4CzIPN impurities were removed via silica plug filtration $\left(\mathrm{SiO}_{2}\right.$, pentane / acetone, 8:2) to give $3 \mathbf{n}(58 \mathrm{mg}, 71 \%)$ as a colourless oil.

$\mathbf{R}_{f} 0.30$ (pentane / $\left.\mathrm{Et}_{2} \mathrm{O}, 1: 1\right)$;

${ }^{1} \mathbf{H}$ NMR $\left(400 \mathrm{MHz}, \mathrm{CDCl}_{3}\right) \delta 7.71-7.63(\mathrm{~d}, J=8.0 \mathrm{~Hz}, 2 \mathrm{H}), 7.35(\mathrm{~d}, J=8.0 \mathrm{~Hz}, 2 \mathrm{H}), 5.92$ (ddt, $J=17.2,10.4,6.0 \mathrm{~Hz}, 1 \mathrm{H}), 5.37$ (dq, $J=17.2,1.5 \mathrm{~Hz}, 1 \mathrm{H}), 5.28(\mathrm{dq}, J=10.4,1.2 \mathrm{~Hz}$, $1 \mathrm{H}), 4.67(\mathrm{dt}, J=6.0,1.2 \mathrm{~Hz}, 2 \mathrm{H}), 4.16(\mathrm{dd}, J=12.0,2.6 \mathrm{~Hz}, 1 \mathrm{H}), 3.78-3.65(\mathrm{~m}, 1 \mathrm{H}), 2.88-$ $2.72(\mathrm{~m}, 2 \mathrm{H}), 2.70(\mathrm{~d}, J=12.0 \mathrm{~Hz}, 1 \mathrm{H}), 2.50(\mathrm{~s}, 1 \mathrm{H}), 2.46-2.36(\mathrm{~m}, 4 \mathrm{H}), 1.93-1.82(\mathrm{~m}, 6 \mathrm{H})$; ${ }^{13} \mathrm{C}$ NMR (101 MHz, $\left.\mathrm{CDCl}_{3}\right) \delta$ 201.7, 168.1, 144.3, 133.3, 131.6, 130.1, 127.8, 119.6, 66.6, 60.7, 51.3, 50.1, 46.4, 44.1, 40.2, 27.9, 21.7;

HRMS (ESI) m/z: [M+Na] ${ }^{+}$calcd for $\mathrm{C}_{21} \mathrm{H}_{25} \mathrm{O}_{5} \mathrm{NSNa} 426.1346$, found 426.1344;

IR (film, $v_{\max } / \mathrm{cm}^{-1}$ ) 2980, 2914, 2878, 1721, 1359. 
2-(Bicyclo[1.1.1]pentan-1-yl)-1-morpholinobutane-1,3-dione, 30<smiles>CC(=O)C(C(=O)N1CCOCC1)C12CCC(C1)C2</smiles>

1-Morpholinobutane-1,3-dione (51 $\mathrm{mg}, 0.30 \mathrm{mmol}, 1.5 \mathrm{eq}$.$) was submitted to General$ Procedure 1. Purification by column chromatography $\left(\mathrm{SiO}_{2}\right.$, pentane / EtOAc, 1:4) gave 30 (37 $\mathrm{mg}, 78 \%$ ) as a pale-yellow solid.

m.p. $47-48{ }^{\circ} \mathrm{C}\left(\mathrm{CH}_{2} \mathrm{Cl}_{2}\right)$

$\mathbf{R}_{f} 0.31$ (pentane / EtOAc, 1:4);

${ }^{1} \mathbf{H}$ NMR (400 MHz, $\left.\mathrm{CDCl}_{3}\right) \delta 3.73$ - $3.52(\mathrm{~m}, 7 \mathrm{H}), 3.47$ - $3.34(\mathrm{~m}, 2 \mathrm{H}), 2.54(\mathrm{~s}, 1 \mathrm{H}), 2.19$ (s, $3 \mathrm{H}), 1.91(\mathrm{~s}, 6 \mathrm{H})$;

${ }^{13} \mathrm{C}$ NMR(101 MHz, $\left.\mathrm{CDCl}_{3}\right) \delta 203.4,166.8,67.0,66.8,59.8,51.3,46.6,42.4,42.3,28.8,28.7$.

Two additional carbon peaks observed due to rotamers.

HRMS (ESI) m/z: $[\mathrm{M}+\mathrm{H}]^{+}$calcd for $\mathrm{C}_{13} \mathrm{H}_{20} \mathrm{O}_{3} \mathrm{~N}$ 238.1438, found 238.1435;

IR (film, $v_{\max } / \mathrm{cm}^{-1}$ ) 2973, 2960, 2909, 2868, 1717, 1619. 
2-(Bicyclo[1.1.1]pentan-1-yl)-3-oxo-N-(thiazol-2-yl)butanamide, 3p<smiles>CC(=O)C(C(=O)Nc1nccs1)C12CCC(C1)C2</smiles>

3-Oxo- $N$-(thiazol-2-yl)butanamide $(55 \mathrm{mg}, 0.30 \mathrm{mmol}, 1.5$ eq.) was submitted to General Procedure 1. Purification by column chromatography $\left(\mathrm{SiO}_{2}\right.$, pentane / EtOAc, $\left.1: 1\right)$ gave 3p (38 $\mathrm{mg}, 77 \%$ ) as a white solid.

m.p. $152-154{ }^{\circ} \mathrm{C}\left(\mathrm{CH}_{2} \mathrm{Cl}_{2}\right)$

$\mathbf{R}_{f} 0.43$ (pentane / EtOAc, 1:1);

${ }^{1} \mathbf{H}$ NMR $\left(400 \mathrm{MHz}, \mathrm{CDCl}_{3}\right) \delta 10.81$ (br. s, $\left.1 \mathrm{H}\right), 7.48(\mathrm{~d}, J=3.6 \mathrm{~Hz}, 1 \mathrm{H}), 6.98(\mathrm{~d}, J=3.6 \mathrm{~Hz}$, $1 \mathrm{H}), 3.91(\mathrm{~s}, 1 \mathrm{H}), 2.56(\mathrm{~s}, 1 \mathrm{H}), 2.30(\mathrm{~s}, 3 \mathrm{H}), 1.86(\mathrm{~s}, 6 \mathrm{H})$;

${ }^{13}$ C NMR (101 MHz, $\left.\mathrm{CDCl}_{3}\right) \delta$ 205.6, 164.7, 157.6, 137.8, 113.9, 61.9, 50.8, 43.0, 31.7, 27.1;

HRMS (ESI) m/z: $[\mathrm{M}+\mathrm{H}]^{+}$calcd for $\mathrm{C}_{12} \mathrm{H}_{15} \mathrm{O}_{2} \mathrm{~N}_{2} \mathrm{~S} 251.0849$, found 251.0847;

IR (film, $v_{\max } / \mathrm{cm}^{-1}$ ) 3169, 2978, 2914, 2876, 1716, 1685, 1567. 
3-Acetyl-1-benzyl-3-(bicyclo[1.1.1]pentan-1-yl)pyrrolidin-2-one, 3q

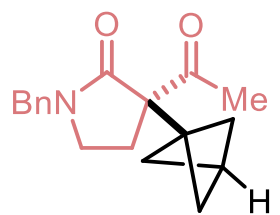

3-Acetyl-1-benzylpyrrolidin-2-one ( $65 \mathrm{mg}, 0.30 \mathrm{mmol}, 1.5 \mathrm{eq}$.) was submitted to General Procedure 1. Purification by column chromatography $\left(\mathrm{SiO}_{2}\right.$, pentane / EtOAc, 9:1) gave 3q (54 $\mathrm{mg}, 95 \%$ ) as a pale-yellow oil.

$\mathbf{R}_{f} 0.36$ (pentane / EtOAc, 7:3);

${ }^{1} \mathbf{H}$ NMR $\left(400 \mathrm{MHz}, \mathrm{CDCl}_{3}\right) \delta 7.37-7.19(\mathrm{~m}, 5 \mathrm{H}), 4.52(\mathrm{~d}, J=14.6 \mathrm{~Hz}, 1 \mathrm{H}), 4.35(\mathrm{~d}, J=14.6$ $\mathrm{Hz}, 1 \mathrm{H}), 3.14-3.03(\mathrm{~m}, 2 \mathrm{H}), 2.57-2.45$ (m, 2H), 2.39 (s, 3H), 1.92 (ddd, $J=13.6,8.1,5.4$ $\mathrm{Hz}, 1 \mathrm{H}), 1.87-1.75(\mathrm{~m}, 6 \mathrm{H})$;

${ }^{13} \mathbf{C}$ NMR(101 MHz, $\left.\mathrm{CDCl}_{3}\right) \delta 204.7,171.1,136.4,128.8,128.3,127.8,63.5,49.5,47.2,46.1$, 43.4, 28.4, 27.3, 24.4;

HRMS (ESI) m/z: $[\mathrm{M}+\mathrm{H}]^{+}$calcd for $\mathrm{C}_{18} \mathrm{H}_{22} \mathrm{O}_{2} \mathrm{~N}$ 284.1645, found 284.1645;

IR (film, $v_{\max } / \mathrm{cm}^{-1}$ ) 2969, 2912, 2874, 1681, 1427. 
3-(bicyclo[1.1.1]pentan-1-yl)-1-methyl-3-nicotinoylpyrrolidin-2-one, 3r

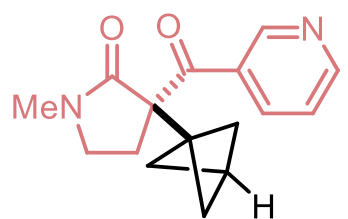

1-Methyl-3-nicotinoylpyrrolidin-2-one (61 mg, $0.30 \mathrm{mmol}, 1.5 \mathrm{eq}$.$) was submitted to General$ Procedure 1. Purification by column chromatography $\left(\mathrm{SiO}_{2}, \mathrm{CH}_{2} \mathrm{Cl}_{2} / \mathrm{MeOH}, 95: 5\right)$ gave $3 \mathbf{r}$ (46 $\mathrm{mg}, 85 \%)$ as a white solid.

m.p. $91-92{ }^{\circ} \mathrm{C}\left(\mathrm{CDCl}_{3}\right)$

$\mathbf{R}_{f} 0.33\left(\mathrm{CH}_{2} \mathrm{Cl}_{2} / \mathrm{MeOH}, 95: 5\right)$;

${ }^{1} \mathbf{H}$ NMR $\left(400 \mathrm{MHz}, \mathrm{CDCl}_{3}\right) \delta 9.06(\mathrm{~s}, 1 \mathrm{H}), 8.75-8.63(\mathrm{~m}, 1 \mathrm{H}), 8.51-8.40(\mathrm{~m}, 1 \mathrm{H}), 7.33$ (dd, $J=7.9,4.7 \mathrm{~Hz}, 1 \mathrm{H}), 3.49-3.36(\mathrm{~m}, 1 \mathrm{H}), 3.36-3.25(\mathrm{~m}, 1 \mathrm{H}), 2.88(\mathrm{~s}, 3 \mathrm{H}), 2.74-2.59$ (m, $1 \mathrm{H}), 2.52(\mathrm{~s}, 1 \mathrm{H}), 2.18(\mathrm{ddd}, J=13.3,8.2,5.0 \mathrm{~Hz}, 1 \mathrm{H}), 1.92-1.76(\mathrm{~m}, 6 \mathrm{H})$;

${ }^{13} \mathbf{C}$ NMR(101 MHz, $\left.\mathrm{CDCl}_{3}\right) \delta 196.7,171.3,152.7,150.4,137.2,133.0,123.1,62.3,49.7,46.8$, 46.4, 30.3, 28.2, 27.2;

HRMS (ESI) calcd for $\mathrm{C}_{16} \mathrm{H}_{19} \mathrm{O}_{2} \mathrm{~N}_{2}[\mathrm{M}+\mathrm{H}]^{+}$271.1441, found 271.1440;

IR (film, $v_{\max } / \mathrm{cm}^{-1}$ ) 2975, 2913, 2872, 1682, 1680. 
2-(Bicyclo[1.1.1]pentan-1-yl)-1,3-diphenylpropane-1,3-dione, 3s<smiles>O=C(C(=O)C(C1CC1)C12CCC1C2)c1ccccc1</smiles>

0.2 mmol scale: 1,3-Diphenylpropane-1,3-dione (67 mg, $0.30 \mathrm{mmol}, 1.5 \mathrm{eq}$.) was submitted to General Procedure 1. Purification by column chromatography $\left(\mathrm{SiO}_{2}\right.$, pentane / EtOAc, 95:5) gave $3 \mathbf{s}$ (48 $\mathrm{mg}, 83 \%)$ as a white solid.

2 mmol scale: To a flame-dried RB flask containing 1,3-diphenylpropane-1,3-dione (670 mg, $3.0 \mathrm{mmol}, 1.5$ eq.), $\mathbf{H 3}$ (56 mg, $0.20 \mathrm{mmol}, 0.1 \mathrm{eq}$ ) and 4CzIPN (39 mg, $2.5 \mathrm{~mol} \%$ ) was added anhydrous $\mathrm{MeCN}(20 \mathrm{~mL})$. The flask was cooled in an ice bath and sparged with argon for 15 min, then [1.1.1]propellane (2.7 $\mathrm{mL}$ of a $0.75 \mathrm{M}$ solution in $\mathrm{Et}_{2} \mathrm{O}, 2.0 \mathrm{mmol}, 1.0$ eq.) was added. The flask was sealed and irradiated with a $440 \mathrm{~nm}$ Kessil PR160L LED lamp for $20 \mathrm{~h}$ with fan cooling then concentrated. Purification by column chromatography $\left(\mathrm{SiO}_{2}\right.$, pentane / $\left.\mathrm{Et}_{2} \mathrm{O}, 7: 3\right)$ gave $3 \mathrm{~s}(500 \mathrm{mg}, 86 \%)$ as a white solid.

m.p. $98-99{ }^{\circ} \mathrm{C}\left(\mathrm{CDCl}_{3}\right)$;

$\mathbf{R}_{f} 0.23$ (pentane / EtOAc, 95:5);

${ }^{1} \mathbf{H}$ NMR $\left(400 \mathrm{MHz}, \mathrm{CDCl}_{3}\right) \delta 7.96-7.89(\mathrm{~m}, 4 \mathrm{H}), 7.60-7.49(\mathrm{~m}, 2 \mathrm{H}), 7.48-7.39(\mathrm{~m}, 4 \mathrm{H})$, $5.47(\mathrm{~s}, 1 \mathrm{H}), 2.53(\mathrm{~s}, 1 \mathrm{H}), 1.94(\mathrm{~s}, 6 \mathrm{H})$;

${ }^{13} \mathbf{C}$ NMR $\left(101 \mathrm{MHz}, \mathrm{CDCl}_{3}\right) \delta 194.6,136.8,133.5,128.9,128.6,57.9,51.6,42.7,29.2$;

HRMS (ESI) m/z: $[\mathrm{M}+\mathrm{H}]^{+}$calcd for $\mathrm{C}_{20} \mathrm{H}_{19} \mathrm{O}_{2}$ 291.1380, found 291.1380;

IR (film, $v_{\max } / \mathrm{cm}^{-1}$ ) 2981, 2968, 2909, 2877, 1696, 1660. 
2-Acetyl-2-(bicyclo[1.1.1]pentan-1-yl)cyclopentan-1-one, 3t

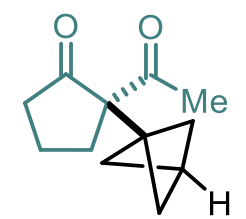

2-Acetylcyclopentan-1-one (38 mg, $0.30 \mathrm{mmol}, 1.5 \mathrm{eq}$.) was submitted to General Procedure 1. Purification by column chromatography $\left(\mathrm{SiO}_{2}\right.$, pentane / EtOAc, 9:1) gave $3 \mathrm{t}$ (27 mg, $71 \%$ ) as a colourless oil.

$\mathbf{R}_{f} 0.40$ (pentane / EtOAc, $4: 1$ );

${ }^{1} \mathbf{H}$ NMR $\left(400 \mathrm{MHz}, \mathrm{CDCl}_{3}\right) \delta 2.57-2.45(\mathrm{~m}, 2 \mathrm{H}), 2.28-2.17(\mathrm{~m}, 5 \mathrm{H}), 1.95-1.85(\mathrm{~m}, 1 \mathrm{H})$, $1.85-1.69(\mathrm{~m}, 8 \mathrm{H})$;

${ }^{13} \mathbf{C}$ NMR $\left(101 \mathrm{MHz}, \mathrm{CDCl}_{3}\right) \delta 214.5,203.1,69.5,49.9,45.9,39.5,29.9,28.2,27.4,19.4$;

HRMS (ESI) m/z: [M+H] $]^{+}$calcd for $\mathrm{C}_{12} \mathrm{H}_{17} \mathrm{O}_{2}$ 193.1223, found 193.1226;

IR (film, $v_{\max } / \mathrm{cm}^{-1}$ ) 2970, 2911, 2875, 1734, 1701. 
1-(Bicyclo[1.1.1]pentan-1-yl)-2-oxocyclopentane-1-carbonitrile, 3u

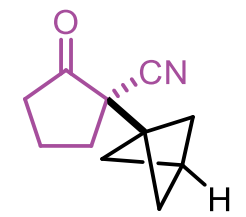

2-Oxocyclopentane-1-carbonitrile (33 $\mathrm{mg}, 0.30 \mathrm{mmol}, 1.5 \mathrm{eq}$.) was submitted to General Procedure 1. Purification by column chromatography $\left(\mathrm{SiO}_{2}\right.$, pentane / EtOAc, 4:1) gave 3u (25 $\mathrm{mg}, 71 \%$ ) as a colourless oil.

$\mathbf{R}_{f} 0.33$ (pentane / EtOAc, 4:1);

${ }^{1} \mathbf{H}$ NMR $\left(400 \mathrm{MHz}, \mathrm{CDCl}_{3}\right) \delta 2.62(\mathrm{~s}, 1 \mathrm{H}), 2.44-2.20(\mathrm{~m}, 4 \mathrm{H}), 2.12-1.91(\mathrm{~m}, 2 \mathrm{H}), 1.86(\mathrm{~s}$, $6 \mathrm{H})$;

${ }^{13} \mathbf{C}$ NMR $\left(101 \mathrm{MHz}, \mathrm{CDCl}_{3}\right) \delta$ 207.9, 118.0, 50.0, 49.1, 43.9, 37.0, 32.5, 27.4, 19.6;

HRMS (ESI) m/z: [M-H] $]^{-}$calcd for $\mathrm{C}_{11} \mathrm{H}_{12} \mathrm{ON}$ 174.0924, found 174.0916;

IR (film, $v_{\max } / \mathrm{cm}^{-1}$ ) 2980, 2915, 2879, 2240, 1749. 
carboxylate, $3 \mathrm{v}$<smiles>COC1(OC)C2CCC1(C1CC1)C(=O)CC1CCC2N1C</smiles>

2-Carbomethoxytropinone (59 mg, $0.30 \mathrm{mmol}, 1.5 \mathrm{eq}$.) was submitted to General Procedure 1. Purification by column chromatography $\left(\mathrm{SiO}_{2}\right.$, pentane / EtOAc, 9:1) gave 3v (42 mg, 74\%) as a white solid.

m.p. $62-64{ }^{\circ} \mathrm{C}\left(\mathrm{CDCl}_{3}\right)$

$\mathbf{R}_{f} 0.48$ (pentane / EtOAc, 4:1);

${ }^{1} \mathbf{H}$ NMR (400 MHz, $\left.\mathrm{CDCl}_{3}\right) \delta 3.69$ (s, 3H), 3.51 - 3.43 (m, 1H), 3.26 (app. t, $J=5.4 \mathrm{~Hz}, 1 \mathrm{H}$ ), $2.57(\mathrm{dd}, J=17.6,5.4 \mathrm{~Hz}, 1 \mathrm{H}), 2.45(\mathrm{~s}, 1 \mathrm{H}), 2.33(\mathrm{~s}, 3 \mathrm{H}), 2.26(\mathrm{dd}, J=17.6,1.6 \mathrm{~Hz}, 1 \mathrm{H}), 2.17$ $-2.06(\mathrm{~m}, 2 \mathrm{H}), 1.91-1.74(\mathrm{~m}, 6 \mathrm{H}), 1.59-1.49(\mathrm{~m}, 2 \mathrm{H})$;

${ }^{13} \mathbf{C}$ NMR(101 MHz, $\left.\mathrm{CDCl}_{3}\right) \delta 203.9,170.0,67.4,67.1,60.9,51.8,50.9,50.0,46.5,41.5,27.0$, 26.9, 25.0;

HRMS (ESI) m/z: [M+H] $]^{+}$calcd for $\mathrm{C}_{15} \mathrm{H}_{22} \mathrm{O}_{3} \mathrm{~N}$ 264.1594, found 264.1590;

IR (film, $v_{\max } / \mathrm{cm}^{-1}$ ) 2981, 2965, 2910, 2877, 1749, 1701. 


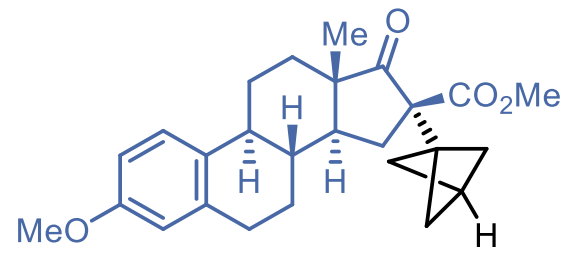

16-carboxymestrone methyl ester $(103 \mathrm{mg}, 0.30 \mathrm{mmol}, 1.5 \mathrm{eq}$.) was submitted to General Procedure 1. Purification by column chromatography $\left(\mathrm{SiO}_{2}\right.$, pentane / EtOAc, 9:1) gave 3w as a single diastereomer $(55 \mathrm{mg}, 67 \%$ ) and a second fraction of $\mathbf{3 w}$ as 1:1 mixture of diastereomers $(10 \mathrm{mg}, 12 \%)$ as a colourless oil.

Total yield $=80 \%, 12: 1 \mathrm{dr}$.

$\mathbf{R}_{f} 0.30$ (pentane / EtOAc, 4:1);

\section{Major diastereoisomer}

${ }^{1} \mathbf{H}$ NMR $\left(400 \mathrm{MHz}, \mathrm{CDCl}_{3}\right) \delta 7.18(\mathrm{~d}, J=8.6 \mathrm{~Hz}, 1 \mathrm{H}), 6.72(\mathrm{dd}, J=8.6,2.8 \mathrm{~Hz}, 1 \mathrm{H}), 6.65(\mathrm{~d}$, $J=2.8 \mathrm{~Hz}, 1 \mathrm{H}), 3.78(\mathrm{~s}, 3 \mathrm{H}), 3.73(\mathrm{~s}, 3 \mathrm{H}), 2.90(\mathrm{dd}, J=8.4,4.0 \mathrm{~Hz}, 2 \mathrm{H}), 2.50(\mathrm{~s}, 1 \mathrm{H}), 2.44-$ $2.34(\mathrm{~m}, 1 \mathrm{H}), 2.26(\mathrm{dd}, J=10.7,4.0 \mathrm{~Hz}, 1 \mathrm{H}), 2.20(\mathrm{~d}, J=10.1 \mathrm{~Hz}, 2 \mathrm{H}), 2.03$ (ddt, $J=12.6$, 5.5, $2.7 \mathrm{~Hz}, 1 \mathrm{H}), 1.99-1.90(\mathrm{~m}, 1 \mathrm{H}), 1.82(\mathrm{~s}, 6 \mathrm{H}), 1.59-1.33(\mathrm{~m}, 5 \mathrm{H}), 0.91(\mathrm{~s}, 3 \mathrm{H})$;

${ }^{13} \mathrm{C}$ NMR $\left(101 \mathrm{MHz}, \mathrm{CDCl}_{3}\right) \delta 211.8,170.6,157.7,137.7,132.0,126.3,114.0,111.7,60.4$, 55.3, 52.4, 49.8, 49.4, 46.43, 46.38, 44.1, 38.1, 31.9, 30.8, 29.6, 27.0, 26.7, 25.8, 14.3;

HRMS (ESI) m/z: [M+H] ${ }^{+}$calcd for $\mathrm{C}_{26} \mathrm{H}_{33} \mathrm{O}_{4} 409.2373$, found 409.2374;

IR (film, $v_{\max } / \mathrm{cm}^{-1}$ ) 2980, 2912, 2872, 1750, 1721. 


\section{B. Further functionalisation}

2-(Bicyclo[1.1.1]pentan-1-yl)-6-methoxy-2,3-dihydro-1H-inden-1-one, 4

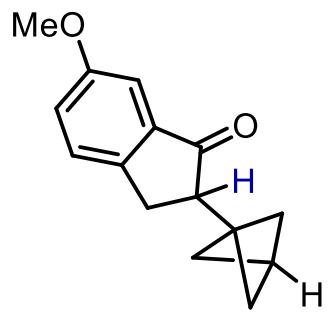

To a solution of $\mathbf{3 g}$ (70 mg, $0.25 \mathrm{mmol})$ in $\mathrm{MeOH} / \mathrm{THF}(1: 1,4 \mathrm{~mL})$ was added $1 \mathrm{M} \mathrm{NaOH}(1$ $\mathrm{mL}, 1.0 \mathrm{mmol}$ ). The resulting solution was maintained at $\mathrm{RT}$ for $16 \mathrm{~h}$ then concentrated in vacuo. Purification by column chromatography $\left(\mathrm{SiO}_{2}\right.$, pentane / $\left.\mathrm{Et}_{2} \mathrm{O}, 9: 1\right)$ afforded 4 (53 mg, 95\%) as a white powder.

m.p. $78-80{ }^{\circ} \mathrm{C}\left(\mathrm{CH}_{2} \mathrm{Cl}_{2}\right)$

$\mathbf{R}_{f} 0.45$ (pentane / $\mathrm{Et}_{2} \mathrm{O}, 4: 1$ );

${ }^{1} \mathbf{H}$ NMR $\left(400 \mathrm{MHz}, \mathrm{CDCl}_{3}\right) \delta 7.39-7.28(\mathrm{~m}, 1 \mathrm{H}), 7.20-7.14(\mathrm{~m}, 2 \mathrm{H}), 3.83(\mathrm{~s}, 3 \mathrm{H}), 3.10$ (dd, $J=16.7,7.5 \mathrm{~Hz}, 1 \mathrm{H}), 2.88-2.78(\mathrm{~m}, 2 \mathrm{H}), 2.50(\mathrm{~s}, 1 \mathrm{H}), 1.88-1.67(\mathrm{~m}, 6 \mathrm{H})$;

${ }^{13} \mathbf{C}$ NMR(101 MHz, $\left.\mathrm{CDCl}_{3}\right) \delta 206.5,159.5,146.8,138.1,127.4,124.2,105.1,55.7,50.1,49.6$, 44.8, 29.5, 28.4;

HRMS (ESI) m/z: [M+H] $]^{+}$calcd for $\mathrm{C}_{15} \mathrm{H}_{17} \mathrm{O}_{2}$ 229.1223, found 229.1221;

IR (film, $v_{\max } / \mathrm{cm}^{-1}$ ) 2969, 2881, 2871, 1699, 1489. 
Ethyl 2-(bicyclo[1.1.1]pentan-1-yl)-2-diazoacetate, 5<smiles></smiles>

To a solution of $3 \mathbf{k}(350 \mathrm{mg}, 1.35 \mathrm{mmol}), p$-ABSA $(651 \mathrm{mg}, 2.70 \mathrm{mmol})$ in anhydrous MeCN $(13 \mathrm{~mL})$ was added DBU (405 $\mu \mathrm{L}, 2.70 \mathrm{mmol})$ in anhydrous $\mathrm{MeCN}(0.5 \mathrm{~mL})$ over $1 \mathrm{~h}$. After a further $2 \mathrm{~h}, p$-ABSA (163 mg, $0.680 \mathrm{mmol})$ was added followed by DBU (100 $\mu \mathrm{L}, 0.680 \mathrm{mmol})$ in anhydrous $\mathrm{MeCN}(0.5 \mathrm{~mL})$ over 10 mins. The resulting solution was stirred at RT for $16 \mathrm{~h}$ then water $(20 \mathrm{~mL})$ and EtOAc $(20 \mathrm{~mL})$ was added. The phases were separated, and the aqueous layer was extracted with EtOAc $(2 \times 50 \mathrm{~mL})$. The combined organic layers were washed with brine $(20 \mathrm{~mL})$ then dried $\left(\mathrm{MgSO}_{4}\right)$, filtered and concentrated in vacuo. Purification by column chromatography $\left(\mathrm{SiO}_{2}\right.$, pentane / $\left.\mathrm{Et}_{2} \mathrm{O}, 9: 1\right)$ afforded $\mathbf{5}(204 \mathrm{mg}, 84 \%)$ as a yellow oil.

$\mathbf{R}_{f} 0.42$ (pentane / $\left.\mathrm{Et}_{2} \mathrm{O}, 9: 1\right)$;

${ }^{1} \mathbf{H}$ NMR $\left(400 \mathrm{MHz}, \mathrm{CDCl}_{3}\right) \delta 4.19(\mathrm{q}, J=7.1 \mathrm{~Hz}, 2 \mathrm{H}), 2.59(\mathrm{~s}, 1 \mathrm{H}), 2.02(\mathrm{~s}, 6 \mathrm{H}), 1.26(\mathrm{t}, J=$ $7.1 \mathrm{~Hz}, 3 \mathrm{H})$;

${ }^{13} \mathrm{C}$ NMR $\left(101 \mathrm{MHz}, \mathrm{CDCl}_{3}\right) \delta 166.9,60.6,52.1,36.9,29.2,14.6\left(\underline{C} \mathrm{~N}_{2}\right.$ not observed $) ;$

HRMS $\left(\mathrm{ESI}^{+}, \mathrm{CI}^{+}, \mathrm{EI}^{+}\right)$: Not found as compound is not sufficiently stable to obtain HRMS or elemental analysis;

IR (film, $v_{\max } / \mathrm{cm}^{-1}$ ) 2981, 2914, 2877, 2079, 1693. 
1-Benzyl-4-(bicyclo[1.1.1]pentan-1-yl)-2,3-dihydro-1H-pyrrole, 6

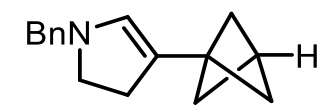

To a solution of 3q $(57 \mathrm{mg}, 0.2 \mathrm{mmol})$ in anhydrous THF $(2 \mathrm{~mL})$ at $0{ }^{\circ} \mathrm{C}$ was added $\mathrm{LiAlH}_{4}$ $\left(150 \mu \mathrm{L}\right.$ of a $4 \mathrm{M}$ solution in $\left.\mathrm{Et}_{2} \mathrm{O}, 0.6 \mathrm{mmol}\right)$. The resulting solution was maintained at $0{ }^{\circ} \mathrm{C}$ for $1 \mathrm{~h}$ then warmed to RT and stirred for a further $1.5 \mathrm{~h}$. The solution was cooled to $0{ }^{\circ} \mathrm{C}$ and $\mathrm{H}_{2} \mathrm{O}$ $(23 \mu \mathrm{L}), 15 \%$ aq. $\mathrm{NaOH}(23 \mu \mathrm{L})$ then $\mathrm{H}_{2} \mathrm{O}(70 \mu \mathrm{L})$ was added, then warmed to RT and stirred for 15 mins. $\mathrm{MgSO}_{4}$ was added and stirred for a further 15 mins, then filtered over Celite. Silica gel was added, and the resulting mixture was concentrated in vacuo. Purification by column chromatography $\left(\mathrm{SiO}_{2}, \mathrm{CH}_{2} \mathrm{Cl}_{2} / \mathrm{MeOH}, 95: 5\right)$ afforded 6 (40 mg, 89\%) as a pale-yellow oil.

$\mathbf{R}_{f} 0.34\left(\mathrm{CH}_{2} \mathrm{Cl}_{2} / \mathrm{MeOH}, 95: 5\right)$;

${ }^{1} \mathbf{H}$ NMR $\left(400 \mathrm{MHz}, \mathrm{CDCl}_{3}\right) \delta 7.36-7.20(\mathrm{~m}, 5 \mathrm{H}), 5.66(\mathrm{t}, J=1.7 \mathrm{~Hz}, 1 \mathrm{H}), 3.79(\mathrm{~s}, 2 \mathrm{H}), 2.96$ $(\mathrm{t}, J=8.9 \mathrm{~Hz}, 2 \mathrm{H}), 2.42(\mathrm{~s}, 1 \mathrm{H}), 2.35(\mathrm{td}, J=8.9,1.7 \mathrm{~Hz}, 2 \mathrm{H}), 1.81(\mathrm{~s}, 6 \mathrm{H})$;

${ }^{13} \mathbf{C ~ N M R}\left(101 \mathrm{MHz}, \mathrm{CDCl}_{3}\right) \delta 138.9,135.5,128.7,128.5,127.2,118.4,58.9,54.3,51.7,43.5$, $30.7,28.2$;

HRMS (ESI) m/z: $[\mathrm{M}+\mathrm{H}]^{+}$calcd for $\mathrm{C}_{16} \mathrm{H}_{20} \mathrm{~N} 226.1590$, found 226.1593;

IR (film, $v_{\max } / \mathrm{cm}^{-1}$ ) 2963, 2907, 2869, 1671, 1205. 
4-(Bicyclo[1.1.1]pentan-1-yl)-3,5-diphenyl-1H-pyrazole, 7<smiles></smiles>

To a solution of $3 \mathbf{s}(29 \mathrm{mg}, 0.10 \mathrm{mmol})$ in $\mathrm{EtOH}(1 \mathrm{~mL})$ was added hydrazine hydrate $(30 \mu \mathrm{L}$, $0.30 \mathrm{mmol}$ ) and the resulting solution was heated at reflux for $4 \mathrm{~h}$. The reaction was cooled to RT and filtered through a plug of silica eluting with 2:1 (pentane / $\mathrm{Et}_{2} \mathrm{O}$ ) to give 7 (27 $\mathrm{mg}, 94 \%$ ) as a white powder.

m.p. $234-236{ }^{\circ} \mathrm{C}\left(\mathrm{CH}_{2} \mathrm{Cl}_{2}\right)$;

$\mathbf{R}_{f} 0.51$ (pentane / EtOAc, 1:1);

${ }^{1} \mathbf{H}$ NMR (400 MHz, $d_{6}$-DMSO) $\delta 12.90(\mathrm{~s}, 1 \mathrm{H}), 7.62-7.25(\mathrm{~m}, 10 \mathrm{H}), 2.28(\mathrm{~s}, 1 \mathrm{H}), 1.80(\mathrm{~s}$, $6 \mathrm{H})$;

${ }^{13}$ C NMR (101 MHz, $d_{6}$-DMSO) $\delta$ 151.3, 141.8, 135.0, 130.4, 129.64, 129.56, 128.4, 128.1, 127.7, 127.5, 115.0, 53.1, 40.4, 29.1;

HRMS (ESI) m/z: [M+H] $]^{+}$calcd for $\mathrm{C}_{20} \mathrm{H}_{19} \mathrm{~N}_{2}$ 287.1543, found 287.1541;

IR (film, $v_{\max } / \mathrm{cm}^{-1}$ ) 3183, 2980, 2966, 2906, 2884. 
4-(Bicyclo[1.1.1]pentan-1-yl)-3,5-diphenylisoxazole, 8<smiles></smiles>

To a solution of $3 \mathbf{s}(29 \mathrm{mg}, 0.10 \mathrm{mmol})$ in $\mathrm{EtOH}(1 \mathrm{~mL})$ was added hydroxyamine hydrochloride (14 mg, $0.20 \mathrm{mmol})$ and $\mathrm{K}_{2} \mathrm{CO}_{3}(28 \mathrm{mg}, 0.2 \mathrm{mmol})$. The resulting solution was heated at reflux for $20 \mathrm{~h}$, then cooled to RT and concentrated. Purification by column chromatography $\left(\mathrm{SiO}_{2}\right.$, pentane / EtOAc, 9:1) afforded $\mathbf{8}(20 \mathrm{mg}, 70 \%)$ as a white powder.

m.p. $147-149{ }^{\circ} \mathrm{C}\left(\mathrm{CH}_{2} \mathrm{Cl}_{2}\right)$;

$\mathbf{R}_{f} 0.60$ (pentane / EtOAc, 9:1);

${ }^{1} \mathbf{H}$ NMR $\left(400 \mathrm{MHz}, \mathrm{CDCl}_{3}\right) \delta 7.70-7.60(\mathrm{~m}, 2 \mathrm{H}), 7.60-7.52(\mathrm{~m}, 2 \mathrm{H}), 7.52-7.43(\mathrm{~m}, 6 \mathrm{H})$, $2.34(\mathrm{~s}, 1 \mathrm{H}), 1.89(\mathrm{~s}, 6 \mathrm{H})$;

${ }^{13} \mathrm{C}$ NMR(101 MHz, $\left.\mathrm{CDCl}_{3}\right) \delta 167.8,164.5,130.2,130.1,129.8,129.5,129.4,128.6,128.4$, 128.3, 114.6, 53.4, 38.7, 30.1;

HRMS (ESI) m/z: [M+H $]^{+}$calcd for $\mathrm{C}_{20} \mathrm{H}_{18} \mathrm{ON} 288.1383$, found 288.1381;

IR (film, $v_{\max } / \mathrm{cm}^{-1}$ ) 2982, 2910, 2878, 1406, 1209. 
Benzyl (S)-3-allyl-3-(bicyclo[1.1.1]pentan-1-yl)-4-oxopiperidine-1-carboxylate, 9<smiles></smiles>

Flask A: To a flame dried flask under argon was added $\mathrm{Pd}_{2} \mathrm{dba}_{3}(5.0 \mathrm{mg}, 2.75 \mathrm{~mol} \%)$ and $(S)$ 4-tert-butyl-2-[2-(diphenylphosphino)phenyl]-2-oxazoline (4.7 mg, $6.00 \mathrm{~mol} \%)$. The flask was placed under an Ar atmosphere via $3 \times$ vacuum / argon cycles. To this flask was added degassed anhydrous toluene (4 mL) and the resulting mixture was stirred at RT for $20 \mathrm{~min}$.

Flask B: In a separate flame dried flask was added 3m (77 mg, $0.20 \mathrm{mmol})$ and degassed anhydrous toluene $(2 \mathrm{~mL})$. The resulting solution was sparged with argon for $10 \mathrm{~min}$ then transferred to Flask A.

The resulting solution was sealed and stirred at RT for $4 \mathrm{~h}$ then purified directly by column chromatography $\left(\mathrm{SiO}_{2}\right.$, pentane / EtOAc, 95:5) to afford $\mathbf{9}(60 \mathrm{mg}, 88 \%, 70 \%$ ee) as a colourless oil.

Racemic material was prepared through an analogous procedure using $\mathrm{Pd}\left(\mathrm{PPh}_{3}\right)_{4}(10 \mathrm{~mol} \%)$.

$\mathbf{R}_{f} 0.38$ (pentane / EtOAc, 4:1);

Very broad signals observed in the ${ }^{1} H$ NMR spectrum and some doubling of peaks observed in the ${ }^{13} C N M R$ spectrum due to rotamers.

$[\alpha]_{D}^{25}=+50.1^{\circ}\left(\mathrm{c}=1.1, \mathrm{CHCl}_{3}\right)$

${ }^{1} \mathbf{H}$ NMR $\left(400 \mathrm{MHz}, \mathrm{CDCl}_{3}\right) \delta 7.47-7.30(\mathrm{~m}, 5 \mathrm{H}), 5.70-5.53(\mathrm{~m}, 1 \mathrm{H}), 5.22-5.10(\mathrm{~m}, 2 \mathrm{H})$, $5.06-4.96(\mathrm{~m}, 2 \mathrm{H}), 4.22-3.85(\mathrm{~m}, 2 \mathrm{H}), 3.40-3.17(\mathrm{~m}, 2 \mathrm{H}), 2.59(\mathrm{dd}, J=14.0,5.8 \mathrm{~Hz}, 1 \mathrm{H})$, $2.53-2.32(\mathrm{~m}, 3 \mathrm{H}), 2.07-1.51(\mathrm{~m}, 7 \mathrm{H})$;

${ }^{13} \mathrm{C}$ NMR (101 MHz, $\left.\mathrm{CDCl}_{3}\right) \delta$ 208.5, 208.2, 155.1, 136.7, 136.4, 133.8, 128.6, 128.3, 128.1, $118.7,67.7,67.5,52.7,52.4,49.0,47.9,47.7,42.5,42.2,40.1,37.0,36.8,26.2$;

HRMS (ESI) m/z: $[\mathrm{M}+\mathrm{H}]^{+}$calcd for $\mathrm{C}_{21} \mathrm{H}_{26} \mathrm{O}_{3} \mathrm{~N}$ 340.1907, found 340.1908;

IR (film, $v_{\max } / \mathrm{cm}^{-1}$ ) 2971, 2910, 2874, 1696, 1631; 
HPLC (CHIRALPAK IC, ethanol $/ n$-hexane $=10 / 90$, flow rate $=0.7 \mathrm{~mL} / \mathrm{min}, \mathrm{I}=220 \mathrm{~nm}) t_{\mathrm{R}}=$ 13.2 min (major), 14.0 min (minor) after derivatization to $(S)$-3-allyl-3-(bicyclo[1.1.1]pentan1-yl)-1-tosylpiperidin-4-one using TMS-I, then TsCl, $\mathrm{Et}_{3} \mathrm{~N}$.

The absolute configuration of the major diastereomer was assigned in analogy to previous literature. ${ }^{4}$

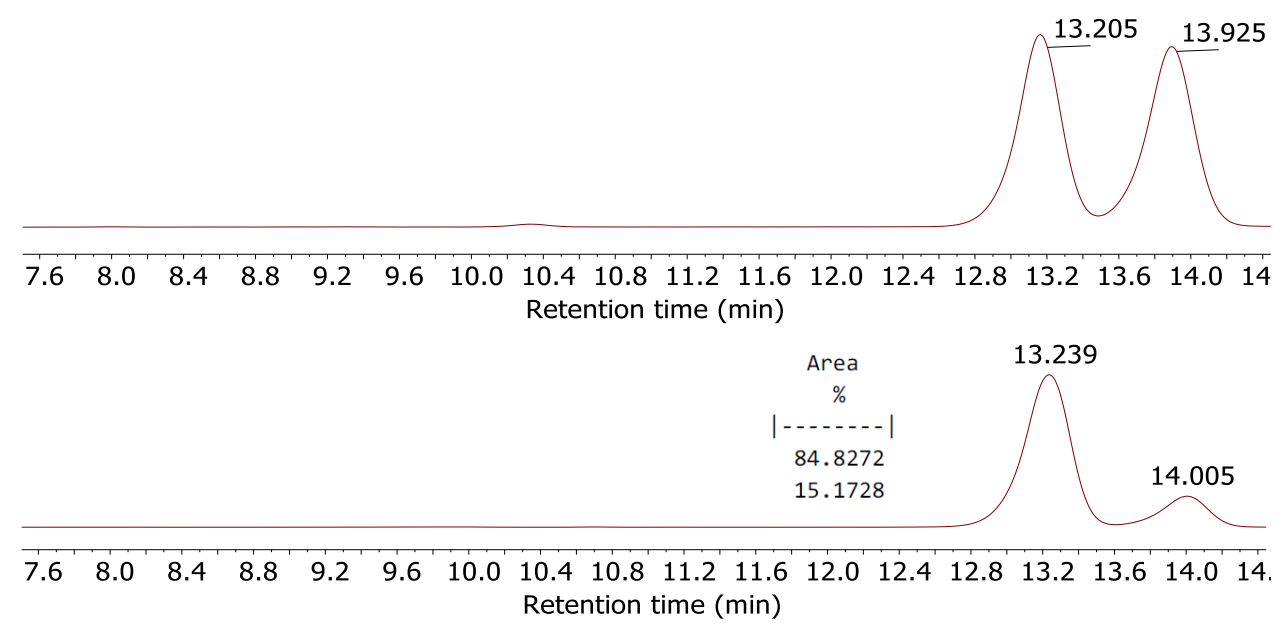


<smiles>C=CC[C@]12CC=CC(C3CC31)C21CC1</smiles>

Flask A: To a flame dried flask under argon was added $\mathrm{Pd}_{2} \mathrm{dba}_{3}(5.0 \mathrm{mg}, 2.75 \mathrm{~mol} \%)$ and $(S)$ 4-tert-butyl-2-[2-(diphenylphosphino)phenyl]-2-oxazoline (4.7 mg, $6.00 \mathrm{~mol} \%)$. The flask was placed under an Ar atmosphere via $3 \times$ vacuum / argon cycles. To this flask was then added degassed anhydrous toluene $(4 \mathrm{~mL})$ and the resulting mixture was stirred at RT for $20 \mathrm{~min}$.

Flask B: In a separate flame dried flask was added 31 (77 $\mathrm{mg}, 0.20 \mathrm{mmol})$ and degassed anhydrous toluene $(2 \mathrm{~mL})$. The resulting solution was sparged with argon for $10 \mathrm{~min}$ then transferred to Flask A.

The resulting solution was sealed and stirred at RT for $4 \mathrm{~h}$ then purified directly by column chromatography $\left(\mathrm{SiO}_{2}\right.$, pentane / $\left.\mathrm{Et}_{2} \mathrm{O}, 95: 5\right)$ to afford $\mathbf{1 0}(46 \mathrm{mg}, 86 \%, 79 \%$ ee) as a white solid.

Racemic material was prepared through an analogous procedure using $\mathrm{Pd}\left(\mathrm{PPh}_{3}\right)_{4}(10 \mathrm{~mol} \%)$. m.p. $69-71{ }^{\circ} \mathrm{C}\left(\mathrm{CH}_{2} \mathrm{Cl}_{2}\right)$;

$\mathbf{R}_{f} 0.29$ (pentane / $\mathrm{Et}_{2} \mathrm{O}, 9: 1$ );

$[\alpha]_{D}^{25}=+2.9^{\circ}\left(\mathrm{c}=0.9, \mathrm{CHCl}_{3}\right)$

${ }^{1} \mathbf{H}$ NMR $\left(400 \mathrm{MHz}, \mathrm{CDCl}_{3}\right) \delta 7.31$ - $7.28(\mathrm{~m}, 1 \mathrm{H}), 7.18$ - $7.13(\mathrm{~m}, 2 \mathrm{H}), 5.52$ (dddd, $J=17.0$, 10.1, 8.7, 6.0 Hz, 1H), 5.05 (dtd, $J=17.0,1.9,1.1 \mathrm{~Hz}, 1 \mathrm{H}), 4.97-4.89$ (m, 1H), 3.82 (s, 3H), $2.94(\mathrm{~d}, J=16.9 \mathrm{~Hz}, 1 \mathrm{H}), 2.89(\mathrm{~d}, J=16.9 \mathrm{~Hz}, 1 \mathrm{H}), 2.64(\mathrm{ddt}, J=13.8,6.0,1.5 \mathrm{~Hz}, 1 \mathrm{H}), 2.46$ (s, $1 \mathrm{H}), 2.21$ (ddt, $J=13.8,8.7,1.1 \mathrm{~Hz}, 1 \mathrm{H}), 1.70-1.58(\mathrm{~m}, 6 \mathrm{H})$;

${ }^{13} \mathrm{C}$ NMR (101 MHz, $\left.\mathrm{CDCl}_{3}\right) \delta 207.9,159.4,146.1,138.2,134.2,127.1,124.2,118.2,105.0$, 55.7, 54.5, 48.6, 48.0, 37.7, 33.3, 26.6;

HRMS (ESI) m/z: [M+H] $]^{+}$calcd for $\mathrm{C}_{18} \mathrm{H}_{21} \mathrm{O}_{2}$ 269.1536, found 269.1537;

IR (film, $v_{\max } / \mathrm{cm}^{-1}$ ) 2961, 2908, 2868, 1698, 1638; 
HPLC (CHIRALPAK IC, isopropanol $/ n$-hexane $=10 / 90$, flow rate $=0.8 \mathrm{~mL} / \mathrm{min}, \mathrm{I}=250 \mathrm{~nm}$ ) $t_{\mathrm{R}}=7.2 \min$ (major), $6.7 \min$ (minor).

The absolute configuration of the major diastereomer was assigned in analogy to previous literature. ${ }^{4}$

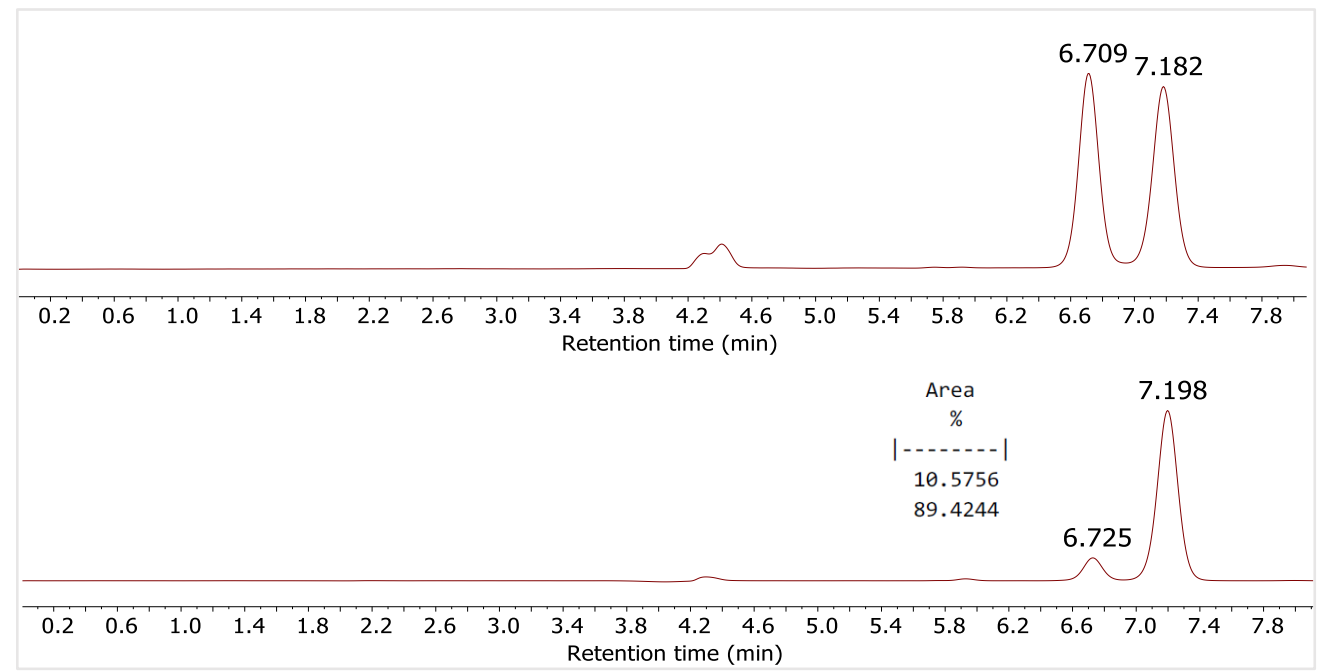




\section{Methyl 7-(((benzyloxy)carbonyl)amino)-3-oxoheptanoate, $2 x$}<smiles>COC(=O)CC(=O)CCCCNC(=O)[O-]</smiles>

Flask A: To a solution of 5-(((benzyloxy)carbonyl)amino)pentanoic acid (3.5 g, $14.0 \mathrm{mmol})$ in anhydrous THF $(15 \mathrm{~mL})$ was added CDI $(2.7 \mathrm{~g}, 16.8 \mathrm{mmol})$ portionwise. The resulting solution was stirred at RT for $16 \mathrm{~h}$.

Flask B: To a flame dried flask at $0{ }^{\circ} \mathrm{C}$ was added $i-\operatorname{PrMgBr}(14.5 \mathrm{~mL}$ of a $2.9 \mathrm{M}$ solution in 2-MeTHF, $42.0 \mathrm{mmol})$. A solution of methyl hydrogen malonate $(2.2 \mathrm{~mL}, 21.0 \mathrm{mmol})$ in anhydrous THF $(20 \mathrm{~mL})$ was then added slowly and the resulting solution was stirred for 30 mins, then warmed to RT and stirred for 30 mins and then heated to $40{ }^{\circ} \mathrm{C}$ and stirred for 30 mins, then cooled back to $0{ }^{\circ} \mathrm{C}$. The solution from Flask A was then added and the resulting solution was stirred at RT for $4 \mathrm{~h}$. The reaction was quenched through dropwise addition of $1 \mathrm{M}$ $\mathrm{HCl}$. After effervescence stopped, $1 \mathrm{M} \mathrm{HCl}(50 \mathrm{~mL})$ was added, and the reaction was stirred for $10 \mathrm{~min}$. The phases were separated, and the aqueous layer was extracted with EtOAc $(3 \times 50$ $\mathrm{mL})$. The combined organic layers were washed with brine $(20 \mathrm{~mL})$ then dried $\left(\mathrm{MgSO}_{4}\right)$, filtered and concentrated in vacuo. Purification by column chromatography $\left(\mathrm{SiO}_{2}\right.$, pentane $/$ EtOAc, 1:1) afforded $\mathbf{2 x}(3.2 \mathrm{~g}, 74 \%)$ as a pale-yellow viscous oil.

$\mathbf{R}_{f} 0.33$ (pentane / EtOAc, 1:1);

${ }^{1} \mathbf{H}$ NMR $\left(400 \mathrm{MHz}, \mathrm{CDCl}_{3}\right) \delta 7.47$ - 7.28 (m, 5H), 5.08 (s, 2H), 4.83 (br. s, 1H), 3.73 (s, 3H), 3.43 (s, 2H), 3.19 (app. q, $J=6.4 \mathrm{~Hz}, 2 \mathrm{H}), 2.57$ (t, $J=7.0 \mathrm{~Hz}, 2 \mathrm{H}), 1.61$ (dt, $J=6.4,5.8 \mathrm{~Hz}$, $2 \mathrm{H}), 1.51(\mathrm{dt}, J=7.0,5.8 \mathrm{~Hz}, 2 \mathrm{H})$;

${ }^{13} \mathrm{C}$ NMR (101 MHz, $\left.\mathrm{CDCl}_{3}\right) \delta 202.5,167.7,156.5,136.7,128.6,128.22,128.21,66.8,52.5$, 49.1, 42.5, 40.7, 29.3, 20.4;

HRMS (ESI) m/z: [M+Na] ${ }^{+}$calcd for $\mathrm{C}_{16} \mathrm{H}_{21} \mathrm{O}_{5} \mathrm{NNa} 330.1312$, found 330.1312;

IR (film, $v_{\max } / \mathrm{cm}^{-1}$ ) 3328, 250, 2883, 1746, 1707, 1683, 1528. 
Methyl 7-(((benzyloxy)carbonyl)amino)-2-(bicyclo[1.1.1]pentan-1-yl)-3-oxoheptanoate, 3x<smiles>COC(=O)C(C(=O)CCCCNC(=O)OCc1ccccc1)C12CCC1C2</smiles>

To a flame-dried flask containing $2 \mathbf{x}$ (1.84 g, $6.0 \mathrm{mmol}, 1.5 \mathrm{eq}.), \mathbf{H 3}$ (111 mg, $0.40 \mathrm{mmol}, 0.1$ eq) and 4CzIPN (78 mg, $2.5 \mathrm{~mol} \%$ ) was added anhydrous MeCN (40 mL). The flask was cooled in an ice bath and sparged with argon for $15 \mathrm{~min}$, then [1.1.1]propellane $(5.3 \mathrm{~mL}$ of a $0.75 \mathrm{M}$ solution in $\mathrm{Et}_{2} \mathrm{O}, 4.0$ mmol, 1.0 eq.) was added. The flask was sealed and irradiated with a 440 $\mathrm{nm}$ Kessil PR160L LED lamp for $20 \mathrm{~h}$ with fan cooling then concentrated. Purification by column chromatography $\left(\mathrm{SiO}_{2}\right.$, pentane / EtOAc, 7:3) afforded 3x $(1.40 \mathrm{~g}, 94 \%)$ as a paleyellow viscous oil.

$\mathbf{R}_{f} 0.50$ (pentane / EtOAc, 1:1);

${ }^{1} \mathbf{H}$ NMR $\left(400 \mathrm{MHz}, \mathrm{CDCl}_{3}\right) \delta 7.38-7.28(\mathrm{~m}, 5 \mathrm{H}), 5.09$ (s, 2H), 4.81 (s, 1H), 3.70 (s, 3H), $3.60(\mathrm{~s}, 1 \mathrm{H}), 3.19$ (app. q, $J=6.5 \mathrm{~Hz}, 2 \mathrm{H}), 2.60-2.41(\mathrm{~m}, 3 \mathrm{H}), 1.86$ (s, 6H), $1.65-1.56$ (m, $2 \mathrm{H}), 1.56-1.35(\mathrm{~m}, 2 \mathrm{H})$;

${ }^{13} \mathrm{C}$ NMR (101 MHz, $\left.\mathrm{CDCl}_{3}\right) \delta$ 203.5, 168.8, 156.5, 136.7, 128.6, 128.21, 128.18, 66.7, 60.7, 52.1, 50.9, 42.2, 41.7, 40.8, 29.4, 28.5, 20.3;

HRMS (ESI) m/z: [M+Na $]^{+}$calcd for $\mathrm{C}_{21} \mathrm{H}_{27} \mathrm{O}_{5} \mathrm{NNa}$ 396.1781, found 396.1782;

IR (film, $v_{\max } / \mathrm{cm}^{-1}$ ) 3349, 2980, 2912, 2875, 1707, 1525. 


\section{BCP Ritalin, 11}<smiles></smiles>

To a flame dried flask under Ar was added Pd/C (10\%, $16 \mathrm{mg})$, followed by a solution of $\mathbf{3 x}$ (112 mg, $0.30 \mathrm{mmol})$ in $\mathrm{MeOH}(3 \mathrm{~mL})$. The resulting solution was evacuated and placed under an atmosphere of $\mathrm{H}_{2}$. This was repeated a further 2 times. The resulting solution was heated at $40{ }^{\circ} \mathrm{C}$ under an atmosphere of $\mathrm{H}_{2}$ for $48 \mathrm{~h}$, then evacuated and placed under an atmosphere of Ar. The solution was filtered over Celite, washing with $\mathrm{MeOH}(3 \times 5 \mathrm{~mL})$, then concentrated in vacuo. Purification by column chromatography $\left(\mathrm{SiO}_{2}, \mathrm{CH}_{2} \mathrm{Cl}_{2} / \mathrm{MeOH}, 9: 1\right)$ afforded 11 (45 $\mathrm{mg}, 67 \%, 6: 1 \mathrm{dr}$ ) as a pale-yellow foam.

$\mathbf{R}_{f} 0.51\left(\mathrm{CH}_{2} \mathrm{Cl}_{2} / \mathrm{MeOH}, 9: 1\right)$;

\section{$\underline{\text { Major diastereomer }}$}

${ }^{1} \mathbf{H}$ NMR $\left(400 \mathrm{MHz}, \mathrm{CDCl}_{3}\right) \delta 3.66(\mathrm{~s}, 3 \mathrm{H}), 3.14-3.06(\mathrm{~m}, 1 \mathrm{H}), 2.80(\mathrm{ddd}, J=10.4,7.8,2.5$ Hz, 1H), 2.65 (app. td, $J=11.7,2.8 \mathrm{~Hz}, 1 \mathrm{H}), 2.57$ (d, $J=7.8 \mathrm{~Hz}, 1 \mathrm{H}), 2.49$ (s, 1H), $1.89-1.69$ $(\mathrm{m}, 7 \mathrm{H}), 1.62-1.54(\mathrm{~m}, 2 \mathrm{H}), 1.48-1.27(\mathrm{~m}, 2 \mathrm{H}), 1.26-1.13(\mathrm{~m}, 1 \mathrm{H})-N-H$ not observed;

${ }^{13} \mathrm{C}$ NMR $\left(101 \mathrm{MHz}, \mathrm{CDCl}_{3}\right) \delta 172.7,57.5,53.2,51.3,51.2,47.0,43.7,30.1,28.0,25.9,24.5$;

\section{$\underline{\text { Minor diastereomer }}$}

${ }^{1} \mathrm{H}$ peaks are obscured by the major diastereomer.

${ }^{13} \mathrm{C}$ NMR $\left(101 \mathrm{MHz}, \mathrm{CDCl}_{3}\right) \delta 173.2,56.7,54.1,51.4$, 51.0, 47.0, 43.6, 31.1, 27.8, 26.0, 24.4;

HRMS (ESI) m/z: [M+H] $]^{+}$calcd for $\mathrm{C}_{13} \mathrm{H}_{22} \mathrm{O}_{2} \mathrm{~N}$ 224.1645, found 224.1646;

IR (film, $v_{\max } / \mathrm{cm}^{-1}$ ) 2967, 2934, 2911, 2872, 2802, 1732. 

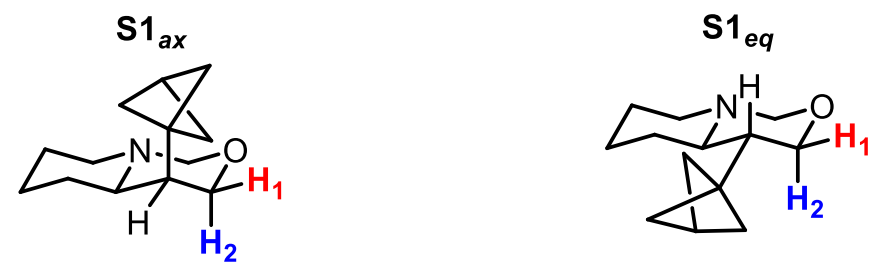

11 was derivatized to $\mathbf{S 1}$ in 2 steps $\left(\mathrm{LiAlH}_{4} ; \mathrm{HCl}\right.$, paraformaldehyde) to determine the stereochemistry of the major diastereomer. The two diastereomers of S1 (S1 $\mathbf{A x}_{\mathbf{a x}}$ and $\left.\mathbf{S 1} \mathbf{1}_{\mathbf{e q}}\right)$ were assigned by analogy to the known 4-methylhexahydro- $1 H, 3 H$-pyrido[1,2-c][1,3]oxazine (S2 $\mathbf{a x}$ and $\mathbf{S 2} \mathbf{e q}_{\mathrm{eq}} .^{5}$ As shown in Figure S6, the $J$ values of $\mathrm{H} 1$ and $\mathrm{H} 2$ of the major diastereomer of S1 are well-matched to the respective $J$ values of $\mathbf{S 2}$ ax, therefore the major diastereomer of $\mathbf{S 1}$ can be assigned as $\mathbf{S} \mathbf{1}_{a x}$. Similarly, the $J$ values of the minor diastereomer of $\mathbf{S 1}$ are well-matched to the respective $J$ values of $\mathbf{S 2}$ eq.

${ }^{1} \mathbf{H}$ NMR $\left(400 \mathrm{MHz}, \mathrm{CDCl}_{3}\right) \delta 3.97(\mathrm{~d}, J=11.1 \mathrm{~Hz}, 1 \mathrm{H}), 3.89(\mathrm{dd}, J=11.3,5.0 \mathrm{~Hz}, 1 \mathrm{H}), 3.66$ $(\mathrm{d}, J=8.1 \mathrm{~Hz}, 1 \mathrm{H}), 3.48(\mathrm{dd}, J=11.1,3.0 \mathrm{~Hz}, 1 \mathrm{H}), 3.44(\mathrm{~d}, J=7.4 \mathrm{~Hz}, 1 \mathrm{H}), 3.19(\mathrm{dd}, J=11.3$ $\mathrm{Hz}, 1 \mathrm{H})$.

HRMS (ESI) m/z: [M+H] ${ }^{+}$calcd for $\mathrm{C}_{13} \mathrm{H}_{22} \mathrm{ON} 208.1696$, found 208.1699.
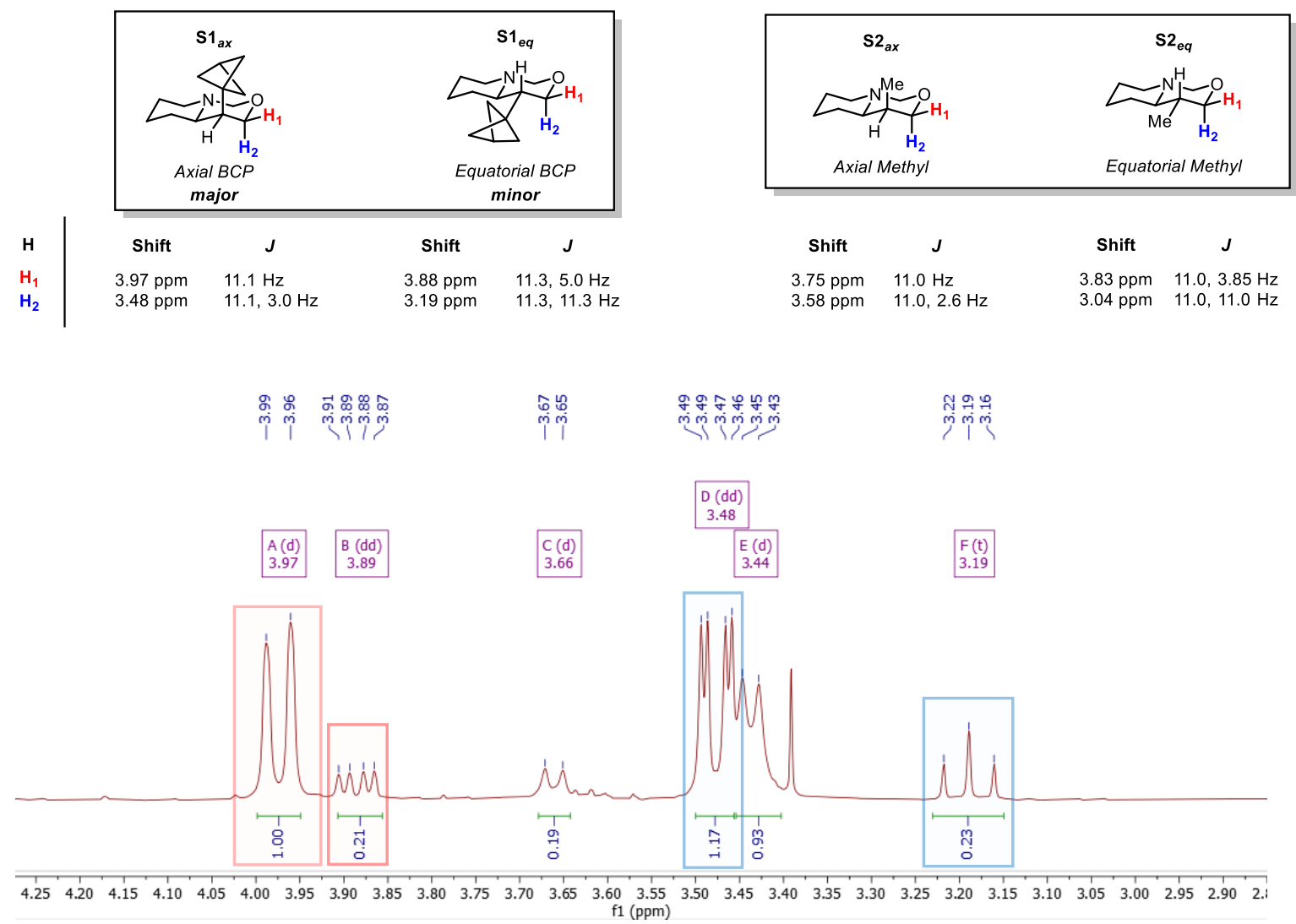

Figure S6. Stereochemical rationale. 


\section{Substrate synthesis}

\section{Dimethyl 4-oxopiperidine-1,3-dicarboxylate, $2 \mathrm{~h}$}<smiles>COC(=O)C1=C(O)CCN(C(C)=O)C1</smiles>

To a solution of methyl 4-oxo-3-piperidinecarboxylate hydrochloride (6.2 g, $31.8 \mathrm{mmol})$ and $\mathrm{Et}_{3} \mathrm{~N}(13 \mathrm{~mL}, 97.0 \mathrm{mmol})$ in $\mathrm{CH}_{2} \mathrm{Cl}_{2}(60 \mathrm{~mL})$ at $0{ }^{\circ} \mathrm{C}$ was slowly added methyl chloroformate ( $3 \mathrm{~mL}, 38.2 \mathrm{mmol})$. The resulting solution was maintained at $0{ }^{\circ} \mathrm{C}$ for $2 \mathrm{~h}$ then quenched with $3 \mathrm{M} \mathrm{HCl}(40 \mathrm{~mL})$. The layers were separated, and the aqueous layer was extracted with $\mathrm{CH}_{2} \mathrm{Cl}_{2}$ $(3 \times 20 \mathrm{~mL})$. The organic layers were combined and washed with brine $(20 \mathrm{~mL})$ and dried with $\mathrm{MgSO}_{4}$. The combined organic layers were washed with brine $(20 \mathrm{~mL})$ then dried $\left(\mathrm{MgSO}_{4}\right)$, filtered and concentrated in vacuo. Purification by column chromatography $\left(\mathrm{SiO}_{2}\right.$, pentane / $\left.\mathrm{Et}_{2} \mathrm{O}, 1: 1\right)$ afforded $\mathbf{2 h}(4.5 \mathrm{~g}, 67 \%, 1: 9$ keto:enol $)$ as a colourless oil.

${ }^{1} \mathbf{H}$ NMR $\left(400 \mathrm{MHz}, \mathrm{CDCl}_{3}\right) \delta 11.96(\mathrm{~s}, 1 \mathrm{H}), 4.15$ - $4.06(\mathrm{~m}, 2 \mathrm{H}), 3.78$ (s, 3H), $3.72(\mathrm{~s}, 3 \mathrm{H})$, $3.65-3.57(\mathrm{~m}, 2 \mathrm{H}), 2.38(\mathrm{t}, J=6.0 \mathrm{~Hz}, 2 \mathrm{H})$.

$\mathbf{2 h}$ is commercially available. 
<smiles>COc1ccc2c(c1)C(=O)C(C(C)=O)C2</smiles>

Prepared according to a literature procedure. ${ }^{6}$

A solution of $\mathrm{NaH}(60 \%$ emulsion, $3.60 \mathrm{~g}, 90.0 \mathrm{mmol})$ in anhydrous THF (40 mL) and dimethylcarbonate $(6.31 \mathrm{~mL}, 75.0 \mathrm{mmol})$. The reaction mixture was cooled to $0{ }^{\circ} \mathrm{C}$ and a solution of 6-methoxyindanone $(4.87 \mathrm{~g}, 30.0 \mathrm{mmol})$ in THF $(40 \mathrm{~mL})$ was added dropwise. The reaction mixture was heated at reflux for $16 \mathrm{~h}$ then cooled to $0{ }^{\circ} \mathrm{C}$ and slowly quenched with a $3 \mathrm{M}$ aqueous solution of $\mathrm{AcOH}(20 \mathrm{~mL})$ and diluted with EtOAc. The layers were separated, and the organic layer was washed with $\mathrm{H}_{2} \mathrm{O}(50 \mathrm{~mL})$ and brine $(50 \mathrm{~mL})$ then dried $\left(\mathrm{MgSO}_{4}\right)$, filtered and concentrated in vacuo. Purification by column chromatography $\left(\mathrm{SiO}_{2}\right.$, pentane / EtOAc, 4:1) afforded a purple solid that could be further purified through recrystallisation from hot hexane / EtOAc to give a $\mathbf{2 g}$ as a white solid (3.30 g, 50\%, 10:1 keto:enol).

${ }^{1} \mathbf{H}$ NMR $\left(400 \mathrm{MHz}, \mathrm{CDCl}_{3}\right) \delta 7.40-7.37(\mathrm{~m}, 1 \mathrm{H}), 7.24-7.18(\mathrm{~m}, 2 \mathrm{H}), 3.83(\mathrm{~s}, 3 \mathrm{H}), 3.79$ (s, $3 \mathrm{H}), 3.76(\mathrm{dd}, J=8.2,3.8 \mathrm{~Hz}, 1 \mathrm{H}), 3.52-3.42(\mathrm{~m}, 1 \mathrm{H}), 3.31(\mathrm{dd}, J=17.2,8.2 \mathrm{~Hz}, 1 \mathrm{H})$.

Analytical data matches that previously reported. ${ }^{6}$ 
<smiles>C=CCOC(=O)C1Cc2ccc(OC)cc2C1=O</smiles>

Prepared according to a literature procedure. ${ }^{7}$

A solution of $\mathbf{2 g}$ (880 mg, $4.0 \mathrm{mmol})$, DMAP (537 mg, $4.4 \mathrm{mmol})$, allyl alcohol (0.82 mL, 12.0 mmol) in anhydrous toluene was heated at reflux for $20 \mathrm{~h}$. The resulting solution was cooled and concentrated in vacuo. Purification by column chromatography $\left(\mathrm{SiO}_{2}\right.$, pentane / EtOAc, 95:5) afforded 21 (620 mg, 63\%, 5:1 keto:enol) as a colourless oil.

${ }^{1} \mathbf{H}$ NMR $\left(200 \mathrm{MHz}, \mathrm{CDCl}_{3}\right) \delta 7.51-7.42(\mathrm{~m}, 1 \mathrm{H}), 7.33-7.25(\mathrm{~m}, 2 \mathrm{H}), 6.01$ (ddt, $J=17.2$, 10.4, $5.7 \mathrm{~Hz}, 1 \mathrm{H}), 5.43$ (dq, $J=17.2,1.4 \mathrm{~Hz}, 1 \mathrm{H}), 5.32$ (dq, $J=10.4,1.4 \mathrm{~Hz}, 1 \mathrm{H}), 4.76$ (app. $\mathrm{dq}, J=5.7,1.4 \mathrm{~Hz}, 2 \mathrm{H}), 3.90$ (s, 3H), $3.84(\mathrm{dd}, J=8.0,4.0 \mathrm{~Hz}, 1 \mathrm{H}), 3.63-3.47$ (m, 1H), 3.37 $(\mathrm{dd}, J=17.0,8.0 \mathrm{~Hz}, 1 \mathrm{H})$.

Analytical data matches that previously reported. ${ }^{8}$ 
<smiles>C=CCOC(=O)C1=C(O)CCN(C(=O)OCc2ccccc2)C1</smiles>

Prepared according to a literature procedure. ${ }^{8}$

To a solution of benzyl 4-oxopiperidine-1-carboxylate (1.9 g, $8.0 \mathrm{mmol}, 1.0$ equiv.) in anhydrous toluene $(12 \mathrm{~mL})$ at $0{ }^{\circ} \mathrm{C}$ was added LiHMDS $(17.6 \mathrm{~mL}, 1.0 \mathrm{M}$ in toluene, $17.6 \mathrm{mmol}$, 2.2 equiv.). After $15 \mathrm{~min}$, allyl chloroformate (1.0 mL, $9.6 \mathrm{mmol}, 1.2$ equiv.) was added and the mixture was warmed to room temperature. After $16 \mathrm{~h}$, a saturated aqueous solution of $\mathrm{NH}_{4} \mathrm{Cl}(24 \mathrm{~mL})$ was added and the phases were separated. The aqueous layer was extracted with EtOAc $(3 \times 8 \mathrm{~mL})$ and the combined organic phases were washed with brine $(16 \mathrm{~mL})$, dried $\left(\mathrm{MgSO}_{4}\right)$, filtered and concentrated in vacuo. Purification by column chromatography $\left(\mathrm{SiO}_{2}\right.$, pentane / EtOAc, 9:1) afforded 2n (1.3 g, 52\%, 1:9 keto:enol) as a pale-yellow oil.

${ }^{1} \mathbf{H}$ NMR $\left(400 \mathrm{MHz}, \mathrm{CDCl}_{3}\right) \delta 11.98(\mathrm{~s}, 1 \mathrm{H}), 7.42$ - 7.30 (m, 5H), 5.94 (ddt, $J=17.2,10.4,5.6$ $\mathrm{Hz}, 1 \mathrm{H}), 5.34(\mathrm{dd}, J=17.2,1.5 \mathrm{~Hz}, 1 \mathrm{H}), 5.26(\mathrm{dq}, J=10.4,1.5 \mathrm{~Hz}, 1 \mathrm{H}), 5.19-5.15(\mathrm{~m}, 2 \mathrm{H})$, 4.69 (dt, $J=5.6,1.5 \mathrm{~Hz}, 2 \mathrm{H}), 4.18$ (s, 2H), 3.65 (app. t, $J=5.9 \mathrm{~Hz}, 2 \mathrm{H}), 2.40$ (br s, 2H).

Analytical data matches that previously reported. ${ }^{8}$ 


\section{1-Tosylpiperidin-4-one}<smiles>CN1CCC(=O)CC1</smiles>

To a solution of 4-piperidone monohydrate hydrochloride (4.6 g, $30 \mathrm{mmol}, 1.0$ equiv.) in $\mathrm{CH}_{2} \mathrm{Cl}_{2}(20 \mathrm{~mL})$ at $0{ }^{\circ} \mathrm{C}$ was added triethylamine $(11.3 \mathrm{~mL}, 81.3 \mathrm{mmol}, 3.0$ equiv.) and tosyl chloride (10.3 g, $54.2 \mathrm{mmol}, 2.0$ equiv.). The resulting mixture was warmed to room temperature and stirred for $16 \mathrm{~h}$. The mixture was quenched with $1 \mathrm{M} \mathrm{HCl}(100 \mathrm{~mL})$ and the aqueous layer extracted with $\mathrm{CH}_{2} \mathrm{Cl}_{2}(3 \times 50 \mathrm{~mL})$. The combined organic layers were washed with brine $(100 \mathrm{~mL})$, dried over $\mathrm{MgSO}_{4}$, filtered, and concentrated in vacuo. Purification by column chromatography $\left(\mathrm{SiO}_{2}\right.$, pentane / EtOAc, 4:1) afforded 1-tosylpiperidin-4-one (4.0 g, $59 \%$ ) as a colorless solid.

${ }^{1} \mathbf{H}$ NMR $\left(400 \mathrm{MHz}, \mathrm{CDCl}_{3}\right) \delta 7.68(\mathrm{~d}, J=8.2 \mathrm{~Hz}, 2 \mathrm{H}), 7.34(\mathrm{~d}, J=8.2 \mathrm{~Hz}, 2 \mathrm{H}), 3.38(\mathrm{t}, J=$ $6.2 \mathrm{~Hz}, 4 \mathrm{H}), 2.53(\mathrm{t}, J=6.2 \mathrm{~Hz}, 4 \mathrm{H}), 2.44(\mathrm{~s}, 3 \mathrm{H})$.

Analytical data matches that previously reported. ${ }^{9}$ 
<smiles>C=CCOC(=O)C1=C(O)CCN(S)C1</smiles>

To a solution of 1-tosylpiperidin-4-one ( $2.0 \mathrm{~g}, 8.0 \mathrm{mmol}, 1.0$ equiv.) in anhydrous toluene (12 $\mathrm{mL}$ ) at $0{ }^{\circ} \mathrm{C}$ was added LiHMDS (17.6 mL, 1.0 M in toluene, $17.6 \mathrm{mmol}, 2.2$ equiv.). After 15 min, allyl chloroformate $(1.0 \mathrm{~mL}, 9.6 \mathrm{mmol}, 1.2$ equiv.) was added and the mixture was warmed to room temperature. After $16 \mathrm{~h}$, a saturated aqueous solution of $\mathrm{NH}_{4} \mathrm{Cl}(24 \mathrm{~mL})$ was added and the phases were separated. The aqueous layer was extracted with EtOAc $(3 \times 8 \mathrm{~mL})$ and the combined organic phases were washed with brine $(16 \mathrm{~mL})$, dried $\left(\mathrm{MgSO}_{4}\right)$, filtered and concentrated in vacuo. Purification by column chromatography $\left(\mathrm{SiO}_{2}\right.$, pentane / EtOAc, 4:1) afforded 2n (1.1 g, 41\%, 5:95 keto:enol) as a pale-yellow oil.

$\mathbf{R}_{f} 0.44$ (pentane / EtOAc, 7:3);

${ }^{1} \mathbf{H}$ NMR $\left(400 \mathrm{MHz}, \mathrm{CDCl}_{3}\right) \delta 11.93(\mathrm{~s}, 1 \mathrm{H}), 7.69(\mathrm{~d}, J=8.2 \mathrm{~Hz}, 2 \mathrm{H}), 7.33(\mathrm{~d}, J=8.2 \mathrm{~Hz}, 2 \mathrm{H})$, $5.93(\mathrm{ddt}, J=17.3,10.4,5.7 \mathrm{~Hz}, 1 \mathrm{H}), 5.43-5.23$ (m, 2H), 4.67 (dt, $J=5.7,1.4 \mathrm{~Hz}, 2 \mathrm{H}), 3.80$ $(\mathrm{t}, J=1.7 \mathrm{~Hz}, 2 \mathrm{H}), 3.27(\mathrm{t}, J=5.9 \mathrm{~Hz}, 2 \mathrm{H}), 2.50-2.41(\mathrm{~m}, 5 \mathrm{H})$;

${ }^{13} \mathrm{C}$ NMR $\left(101 \mathrm{MHz}, \mathrm{CDCl}_{3}\right) \delta 170.0,169.8,144.0,133.6,131.8,129.9,127.8,118.9,95.1$, 65.4, 42.4, 42.3, 29.1, 21.7;

IR (thin film, $v_{\max } / \mathrm{cm}^{-1}$ ) 2981, 1667, 1308, 1227, 1164;

HRMS (ESI) m/z: $[\mathrm{M}+\mathrm{H}]^{+}$calcd for $\mathrm{C}_{16} \mathrm{H}_{19} \mathrm{NO}_{5} \mathrm{~S} 338.1057$, found 338.1058. 


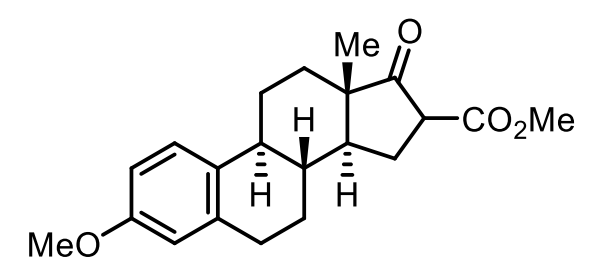

Prepared according to a literature procedure. ${ }^{10}$

A solution of $\mathrm{NaH}(60 \%$ emulsion, $291 \mathrm{mg}, 7.3 \mathrm{mmol})$ in anhydrous THF $(7 \mathrm{~mL})$ and dimethylcarbonate $(0.51 \mathrm{~mL}, 6.1 \mathrm{mmol})$ was heated to reflux and a solution of mestrone (690 $\mathrm{mg}, 2.4 \mathrm{mmol})$ in anhydrous THF $(7 \mathrm{~mL})$ was added dropwise. The resulting mixture was refluxed for $16 \mathrm{~h}$ and then cooled to $0{ }^{\circ} \mathrm{C}$ and slowly quenched with a $3 \mathrm{M}$ aqueous solution of $\mathrm{AcOH}(20 \mathrm{~mL})$ and diluted with EtOAc. The layers were separated and the aqueous layer was extracted with EtOAc $(2 \times 20 \mathrm{~mL})$ and the combined organic layers were washed with $\mathrm{H}_{2} \mathrm{O}(50$ $\mathrm{mL})$ and brine $(50 \mathrm{~mL})$ then dried $\left(\mathrm{MgSO}_{4}\right)$, filtered and concentrated in vacuo. Purification by column chromatography $\left(\mathrm{SiO}_{2}\right.$, pentane / EtOAc, 4:1) afforded $\mathbf{2 w}$ as a white solid $(1.9 \mathrm{~g}, 78 \%)$.

${ }^{1} \mathbf{H}$ NMR $\left(400 \mathrm{MHz}, \mathrm{CDCl}_{3}\right) \delta 10.68(\mathrm{~d}, J=8.7 \mathrm{~Hz}, 1 \mathrm{H}), 10.20(\mathrm{dd}, J=8.6,2.8 \mathrm{~Hz}, 1 \mathrm{H}), 10.13$ (d, $J=2.8 \mathrm{~Hz}, 1 \mathrm{H}), 7.26$ (s, 3H), 7.24 (s, 3H), 6.69 (dd, $J=9.9,8.4 \mathrm{~Hz}, 1 \mathrm{H}), 6.43-6.34$ (m, $2 \mathrm{H}), 5.93-5.70(\mathrm{~m}, 3 \mathrm{H}), 5.62-5.41(\mathrm{~m}, 3 \mathrm{H}), 5.16-4.87(\mathrm{~m}, 5 \mathrm{H}), 4.46(\mathrm{~s}, 3 \mathrm{H})$.

Analytical data matches that previously reported. 


\section{Spectra}

3-Acetyl-3-(bicyclo[1.1.1]pentan-1-yl)dihydrofuran-2(3H)-one, 3a

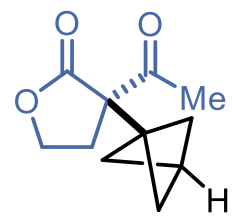

${ }^{1} \mathbf{H}$ NMR (400 MHz, $\left.\mathrm{CDCl}_{3}\right)$

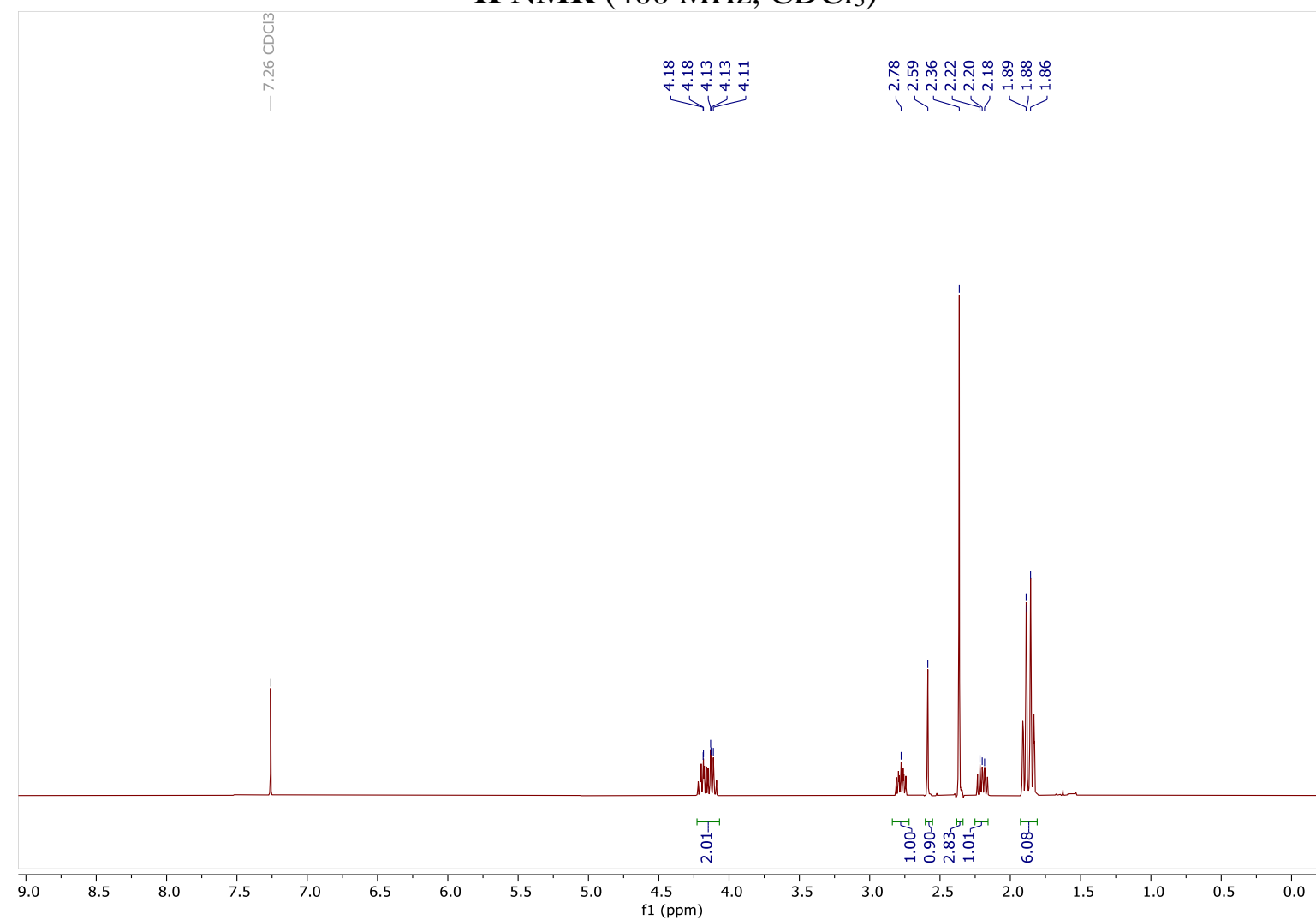

${ }^{13}$ C NMR $\left(101 \mathrm{MHz}, \mathrm{CDCl}_{3}\right)$

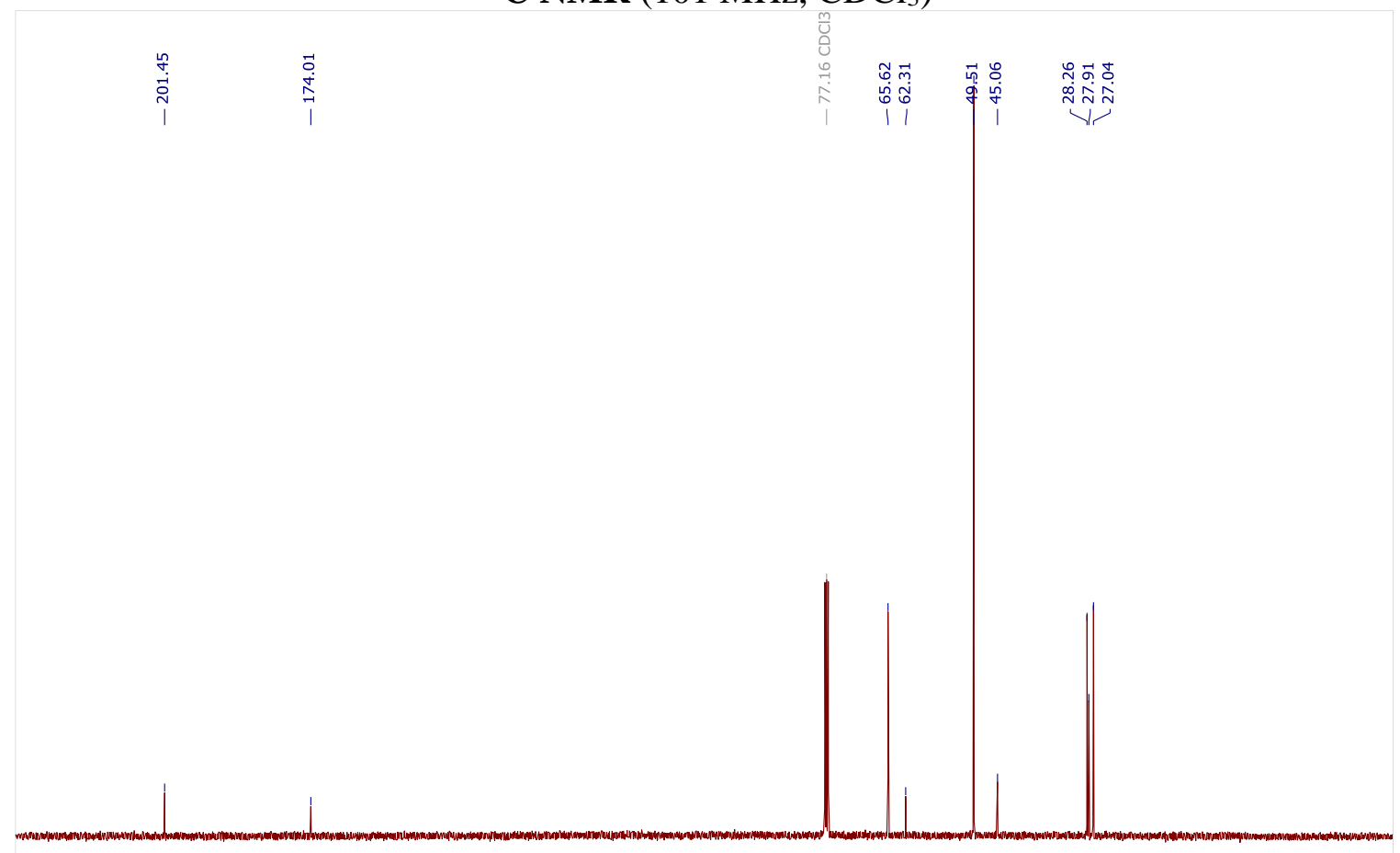

$\begin{array}{llllllllllllllllllllllllllll}1 & 1 \\ 220 & 210 & 200 & 190 & 180 & 170 & 160 & 150 & 140 & 130 & 120 & 110 & 100 & 90 & 80 & 70 & 60 & 50 & 40 & 30 & 20 & 10 & 0 & -10 & -20\end{array}$ 
Methyl 1-(bicyclo[1.1.1]pentan-1-yl)-2-oxocyclopentane-1-carboxylate, 3b

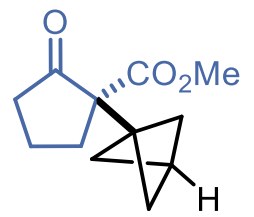

${ }^{1} \mathbf{H}$ NMR (400 MHz, $\left.\mathrm{CDCl}_{3}\right)$

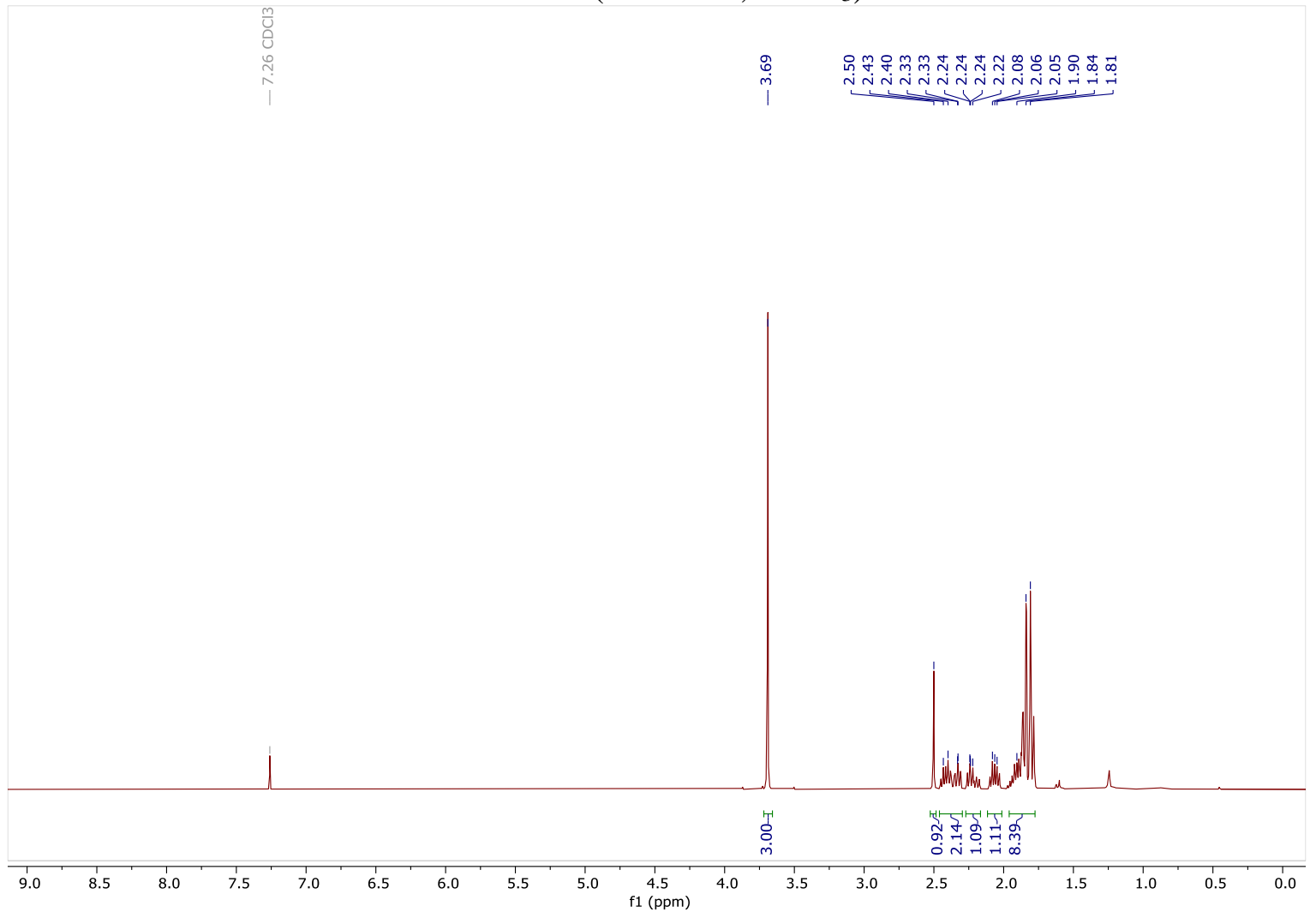

${ }^{13}$ C NMR (101 MHz, $\left.\mathrm{CDCl}_{3}\right)$

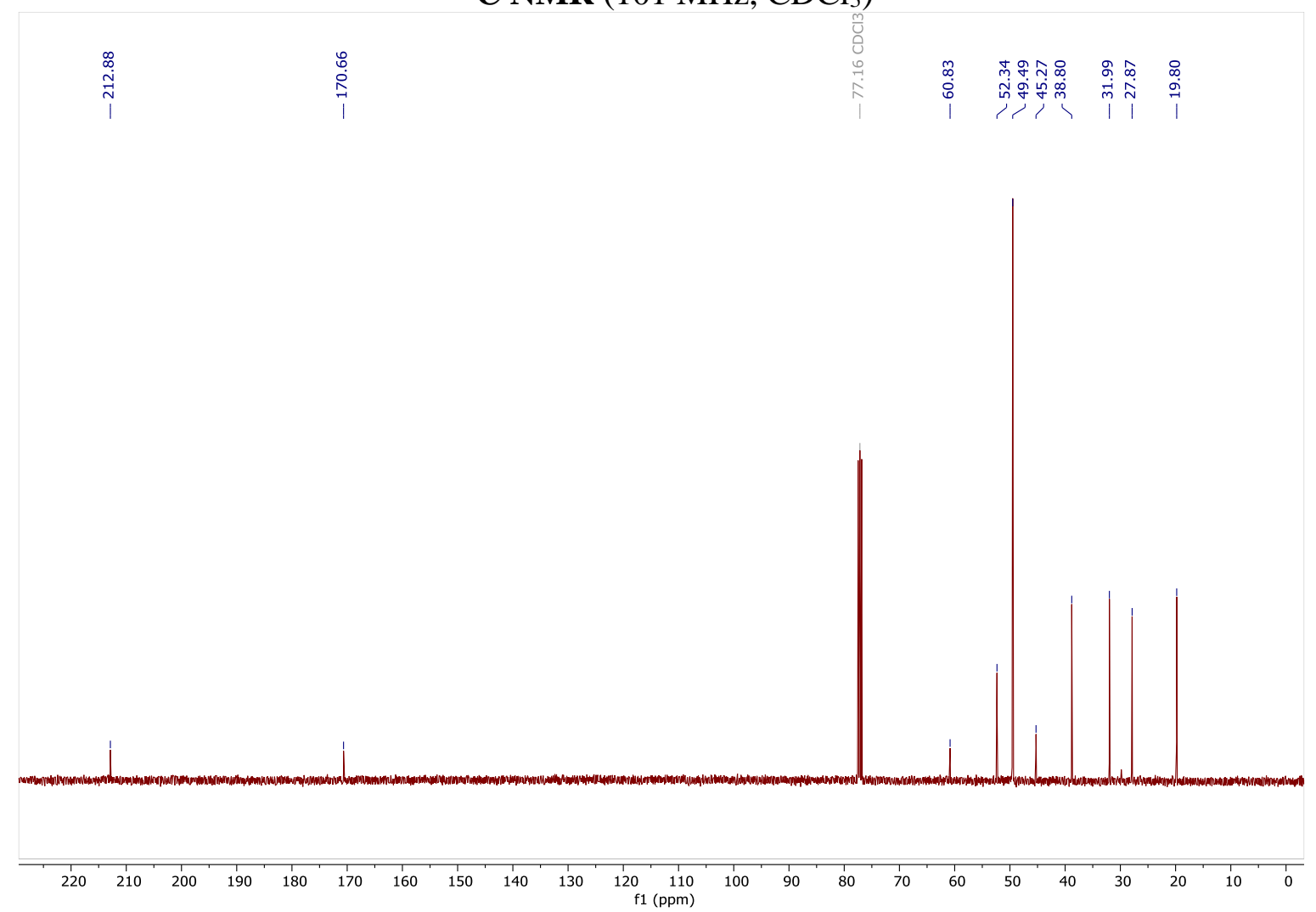


Methyl 3-(bicyclo[1.1.1]pentan-1-yl)-4-oxo-1-phenylpyrrolidine-3-carboxylate, 3c

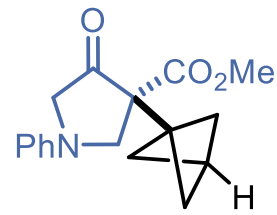

${ }^{1} \mathbf{H}$ NMR $\left(400 \mathrm{MHz}, \mathrm{CDCl}_{3}\right)$

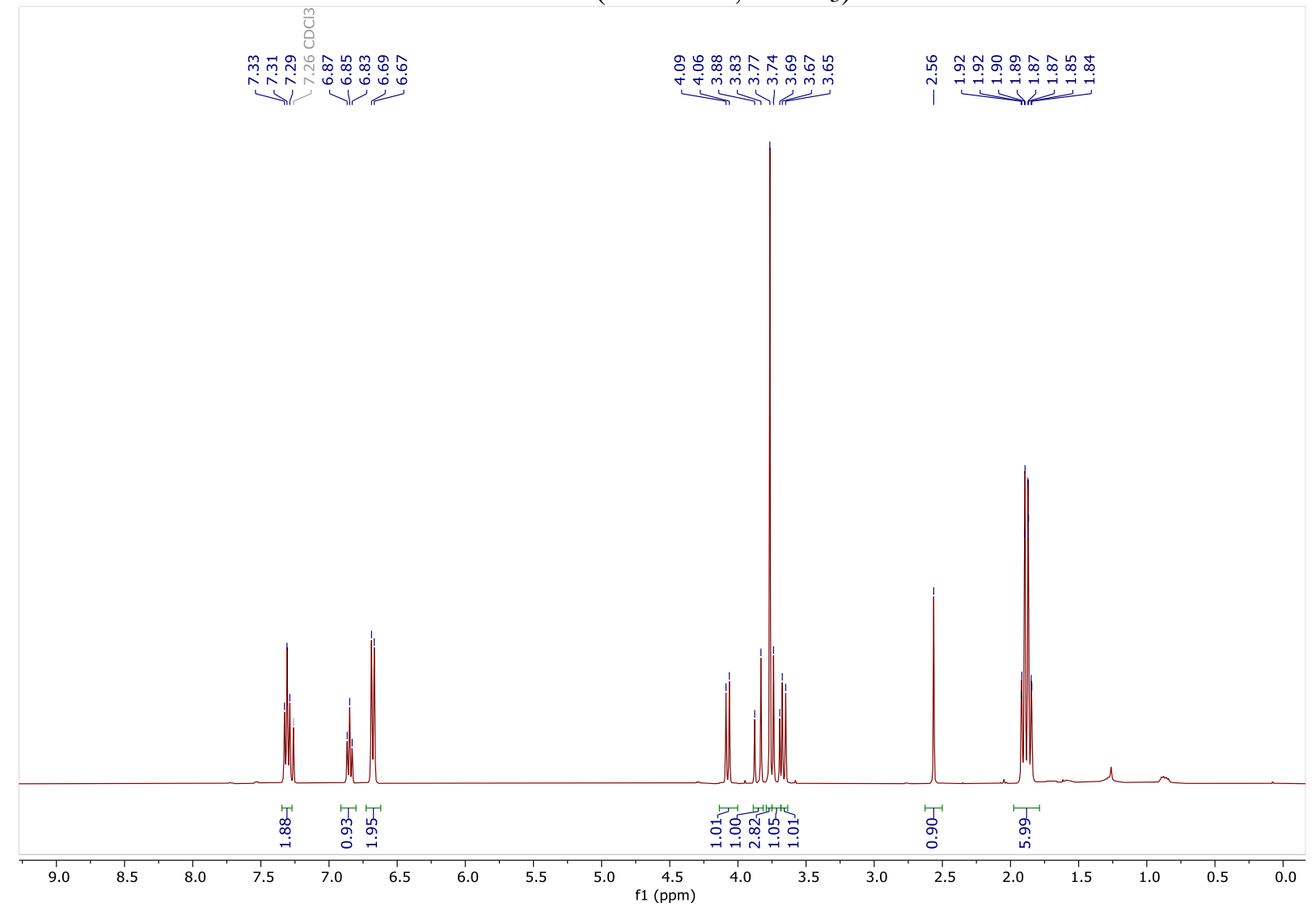

${ }^{13} \mathbf{C}$ NMR $\left(101 \mathrm{MHz}, \mathrm{CDCl}_{3}\right)$

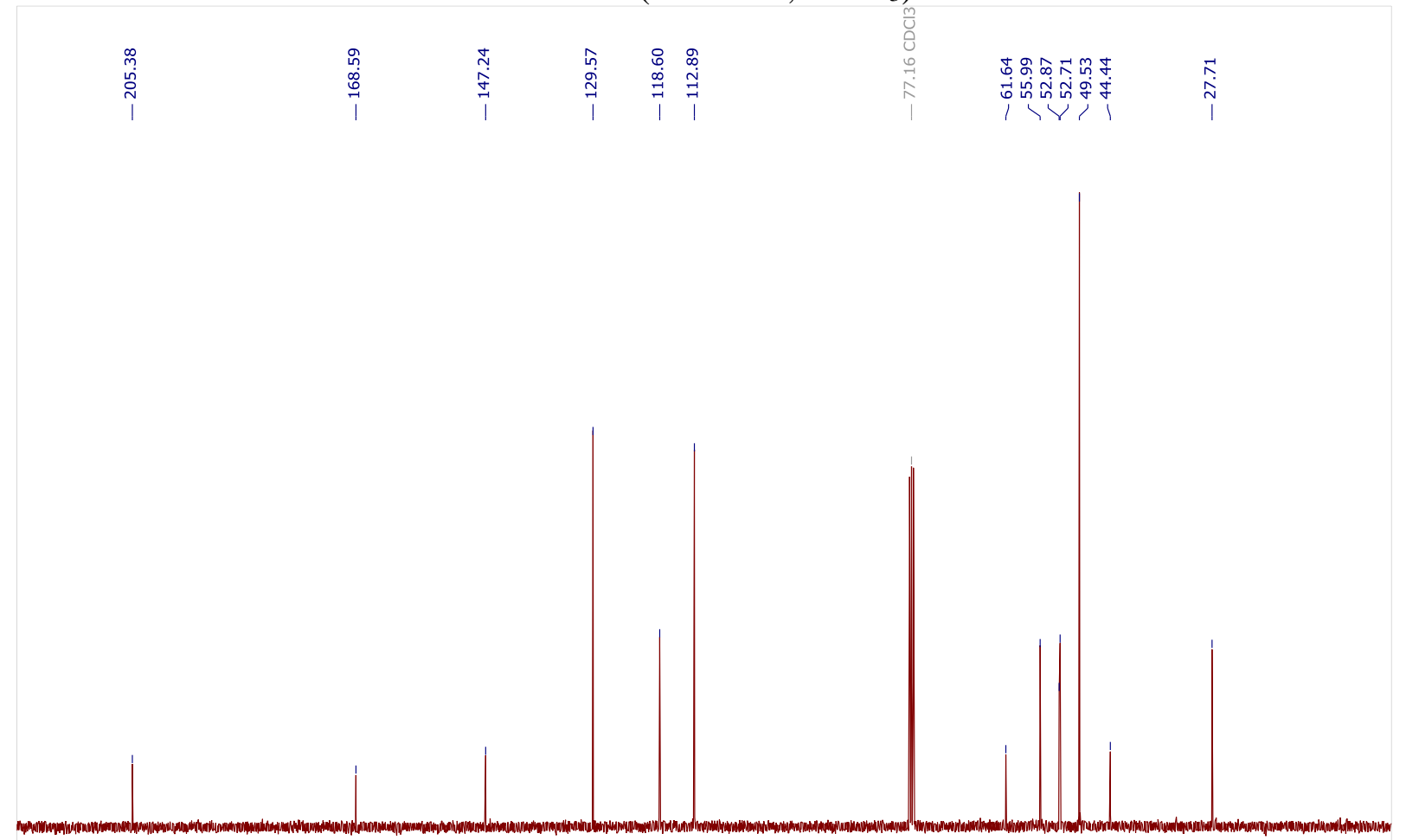

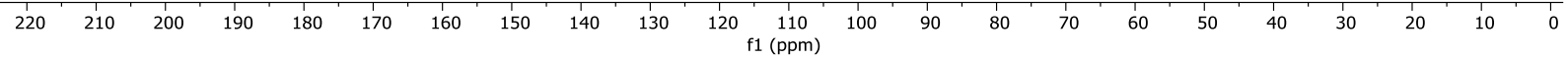


3-(bicyclo[1.1.1]pentan-1-yl)-3-isonicotinoyldihydrofuran-2(3H)-one, 3d

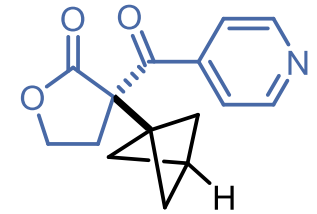

${ }^{1} \mathbf{H}$ NMR $\left(400 \mathrm{MHz}, \mathrm{CDCl}_{3}\right)$

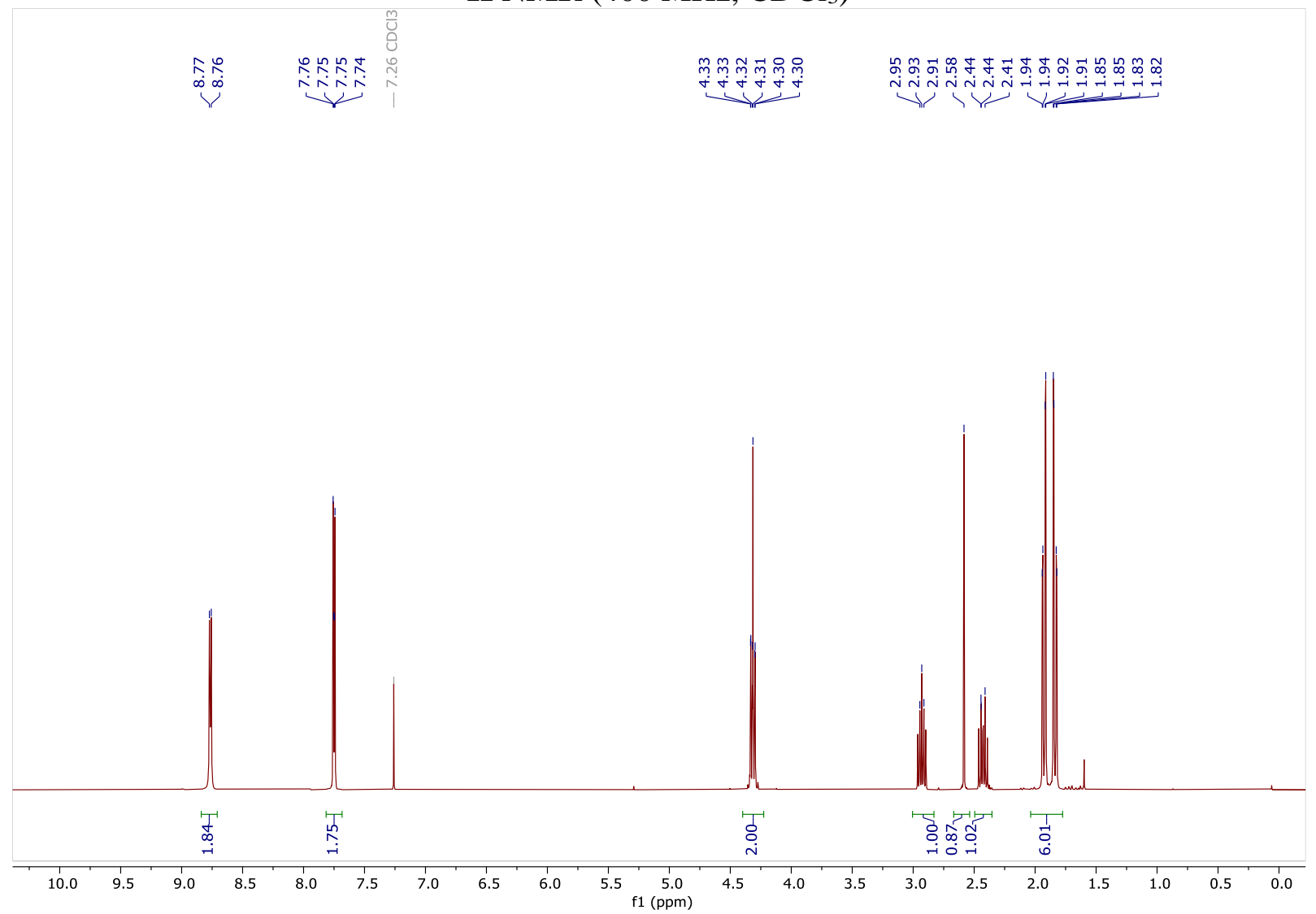

${ }^{13} \mathbf{C}$ NMR $\left(101 \mathrm{MHz}, \mathrm{CDCl}_{3}\right)$

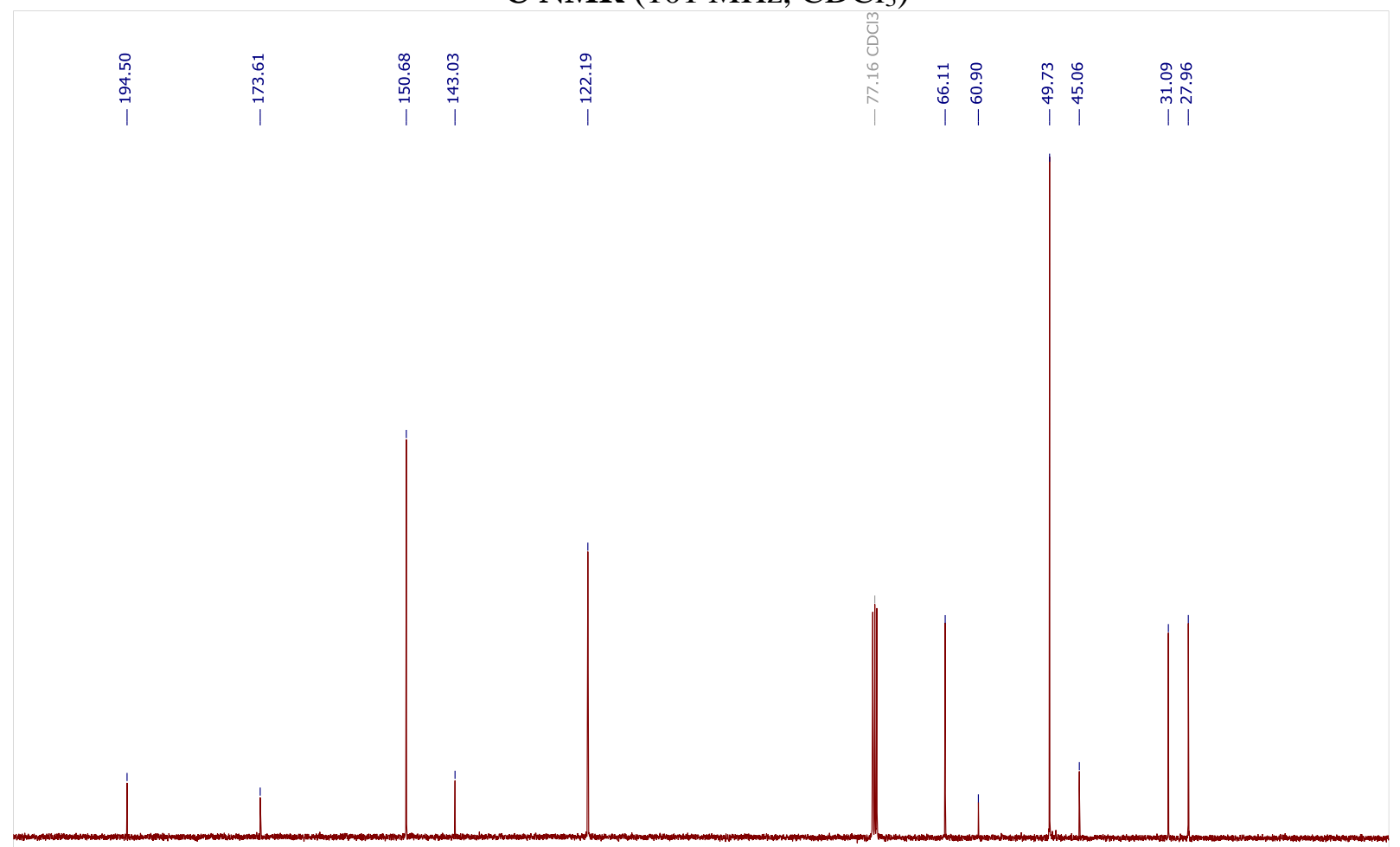

$\begin{array}{lllllllllll}210 & 200 & 190 & 180 & 170 & 160 & 150 & 140 & 130 & 120 & \begin{array}{c}110 \\ \mathrm{f} 1(\mathrm{ppm})\end{array}\end{array}$ 
Ethyl 1-benzyl-3-(bicyclo[1.1.1]pentan-1-yl)-4,5-dioxopyrrolidine-3-carboxylate, 3e

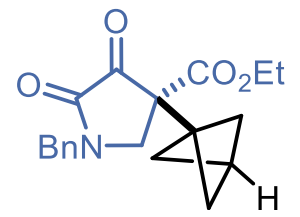

${ }^{\mathbf{1}} \mathbf{H}$ NMR (400 MHz, $\mathrm{CDCl}_{3}$ )

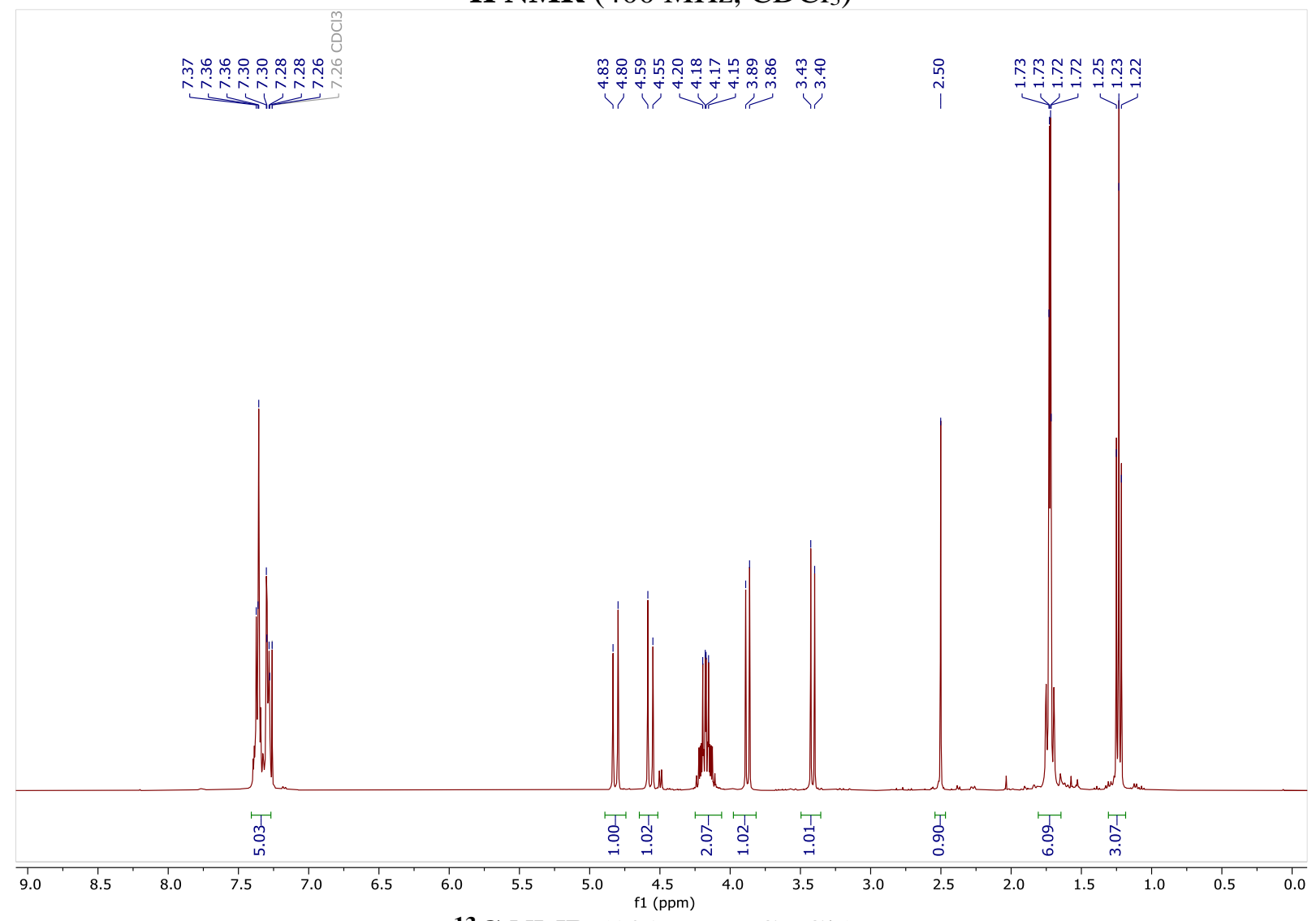

${ }^{13} \mathrm{C}$ NMR $\left(101 \mathrm{MHz}, \mathrm{CDCl}_{3}\right)$

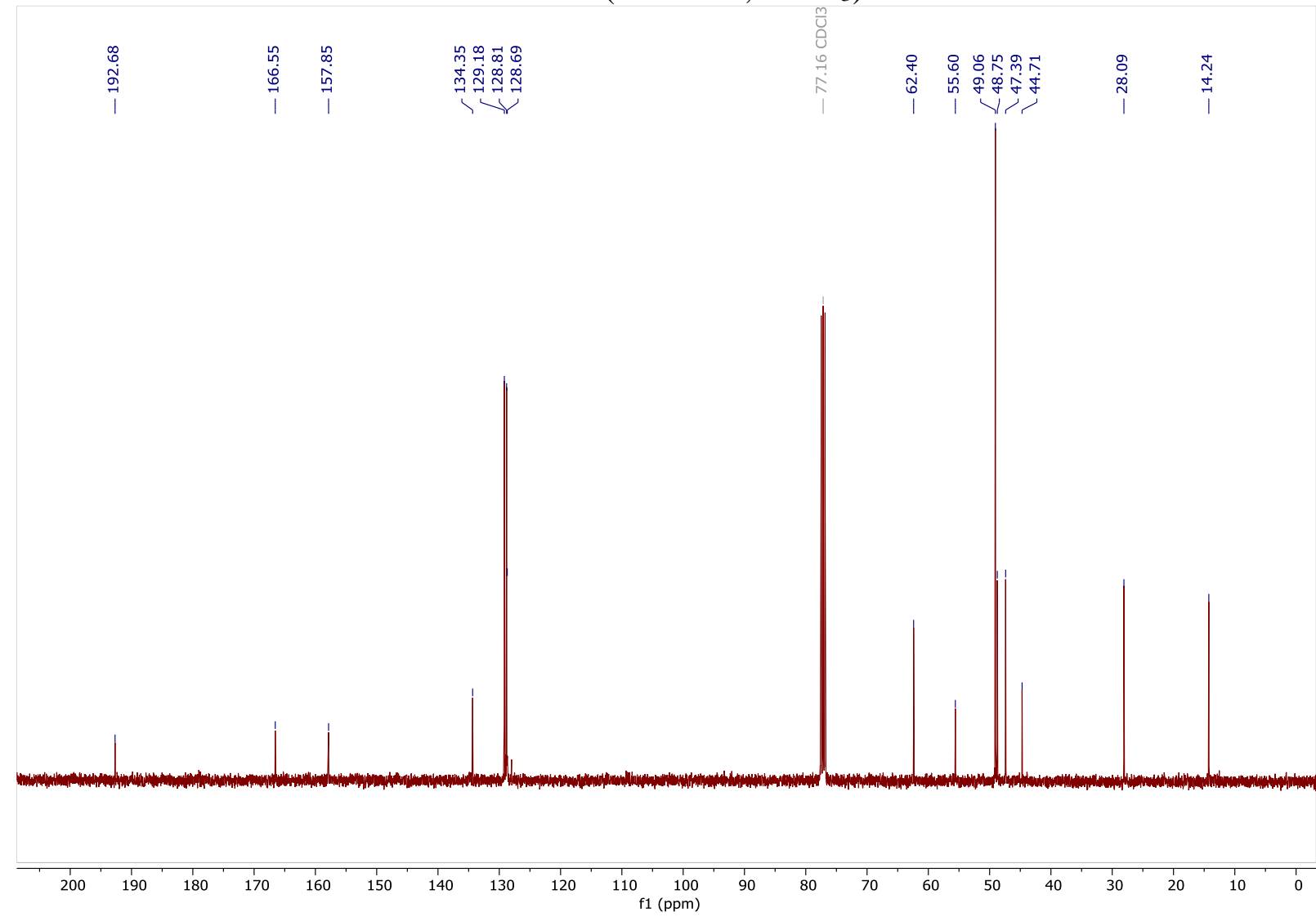


Methyl 2-(bicyclo[1.1.1]pentan-1-yl)-6-methyl-1-oxo-2,3-dihydro-1H-indene-2carboxylate, $3 f$

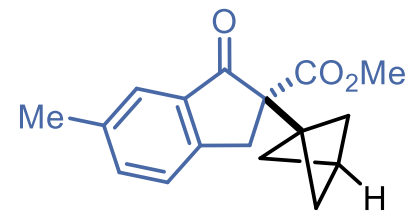

${ }^{1} \mathbf{H}$ NMR $\left(400 \mathrm{MHz}, \mathrm{CDCl}_{3}\right)$

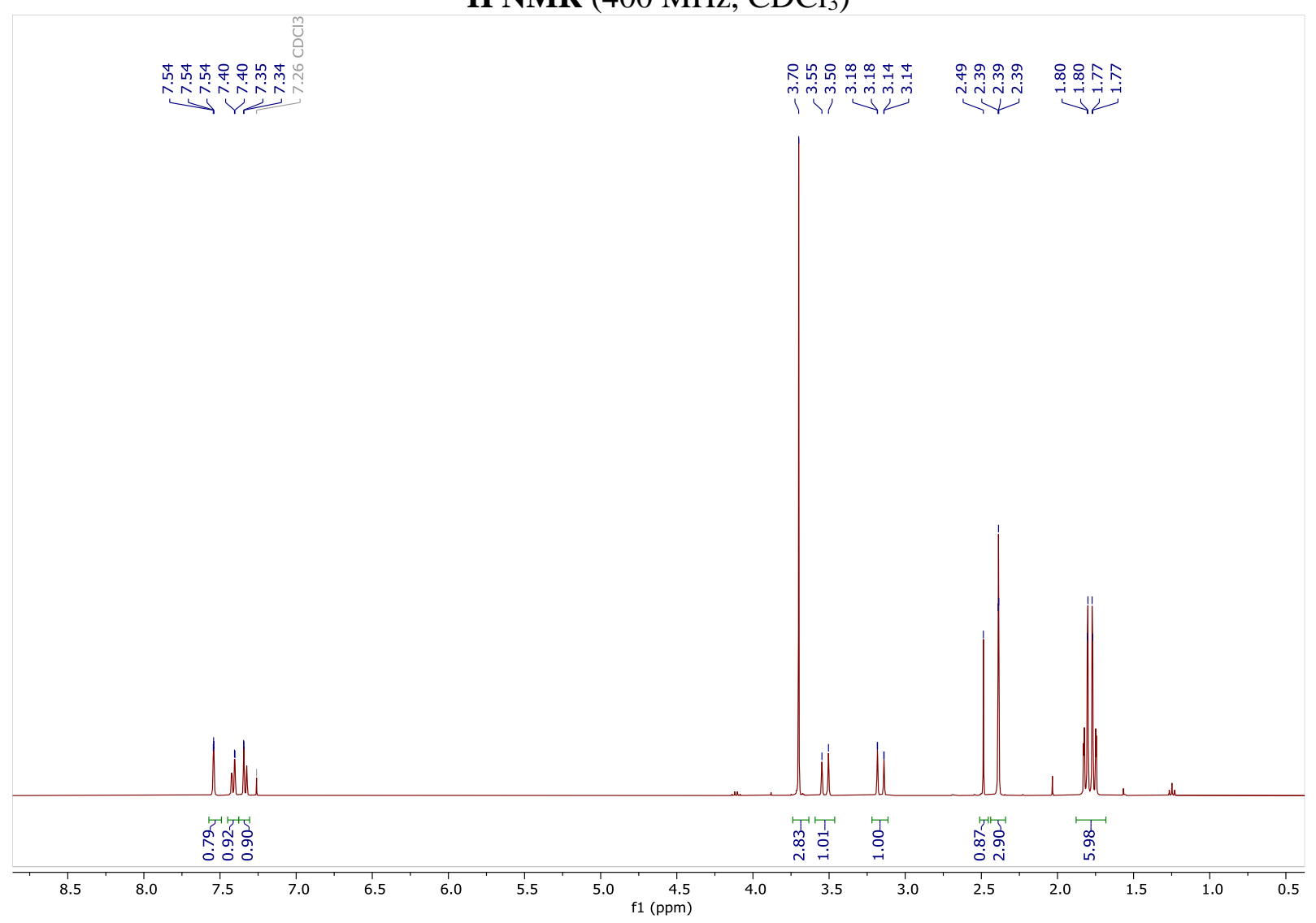

${ }^{13} \mathrm{C}$ NMR $\left(101 \mathrm{MHz}, \mathrm{CDCl}_{3}\right)$

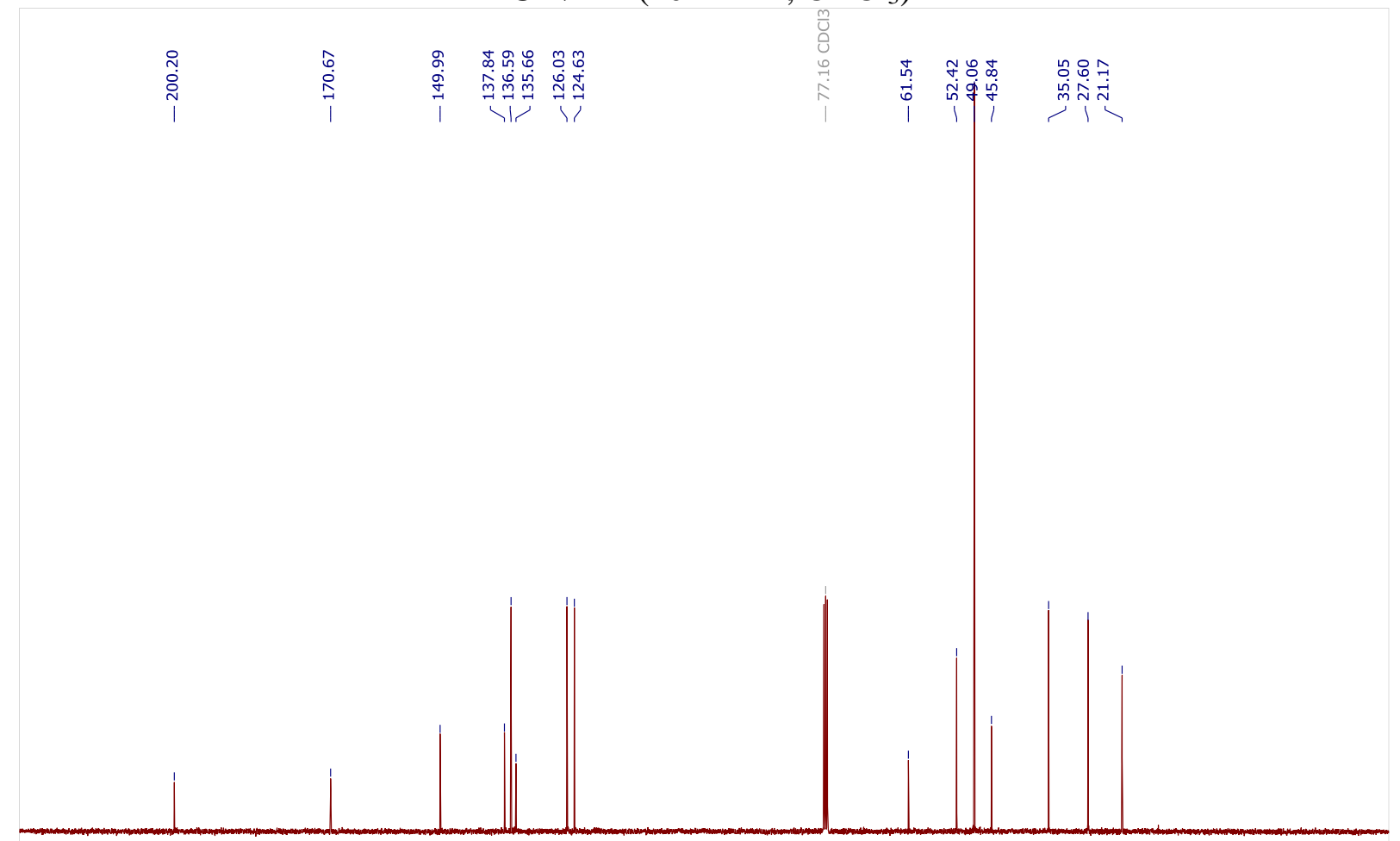

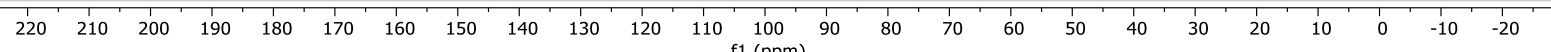



carboxylate, $3 g$

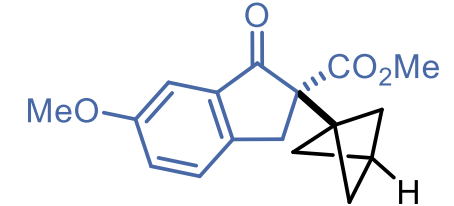

${ }^{\mathbf{1}} \mathbf{H}$ NMR (400 MHz, $\left.\mathrm{CDCl}_{3}\right)$

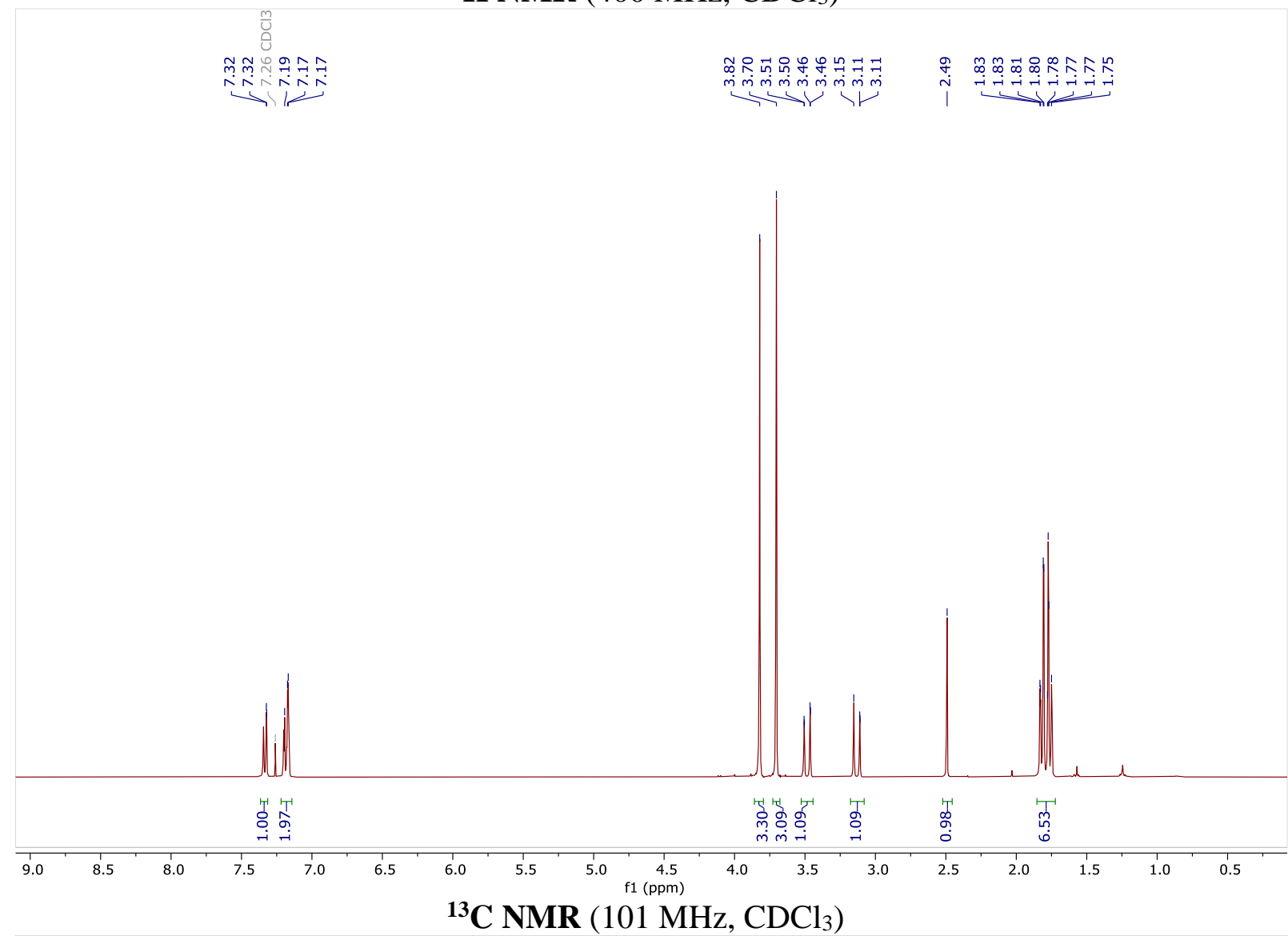

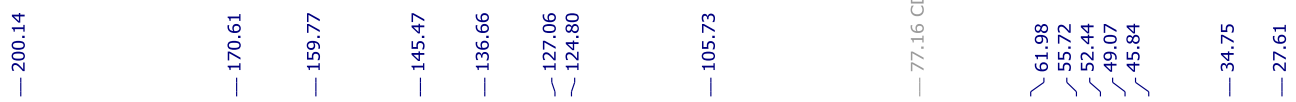


Dimethyl 3-(bicyclo[1.1.1]pentan-1-yl)-4-oxopiperidine-1,3-dicarboxylate, $3 \mathrm{~h}$

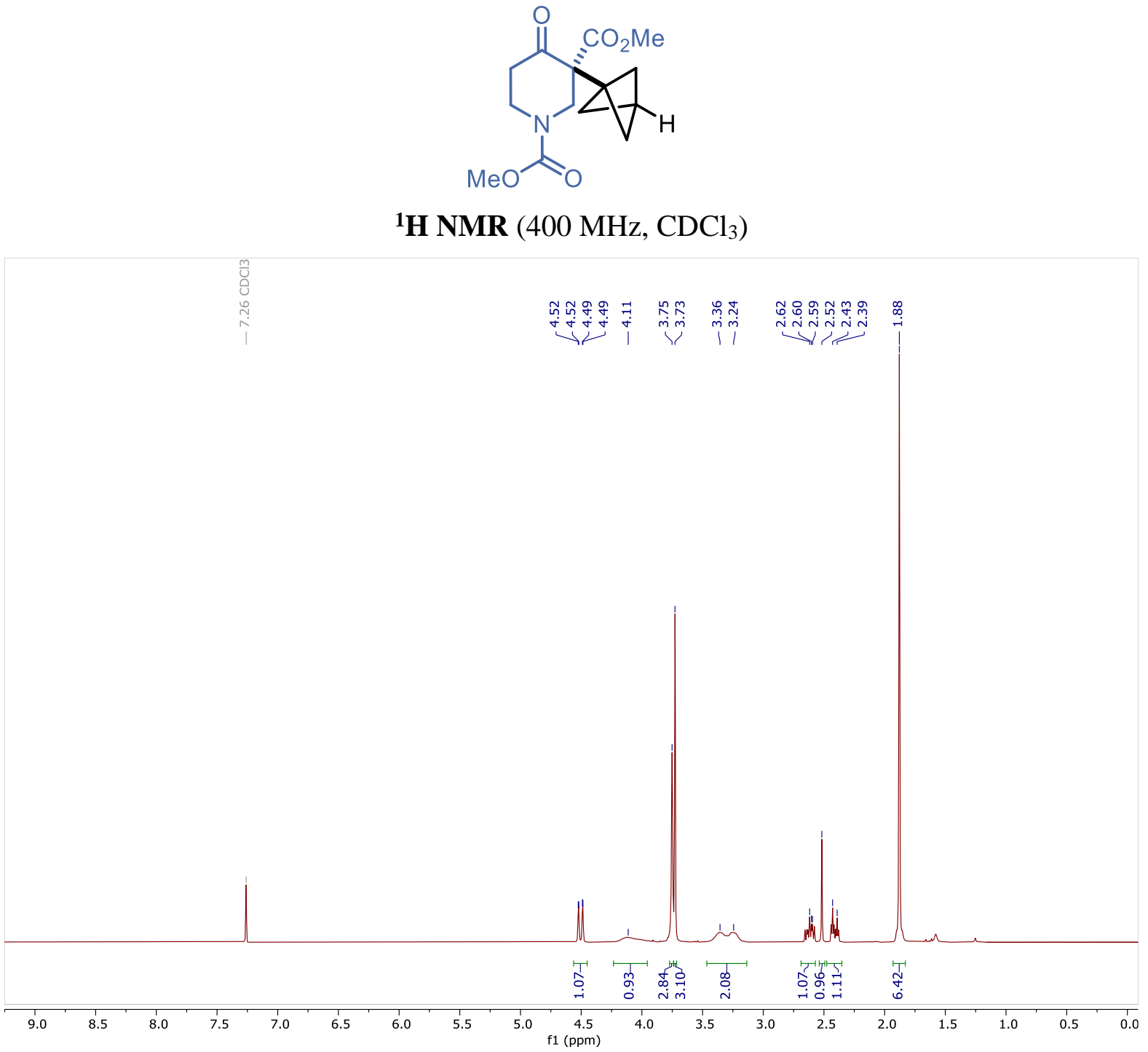

${ }^{13} \mathbf{C}$ NMR $\left(101 \mathrm{MHz}, \mathrm{CDCl}_{3}\right)$

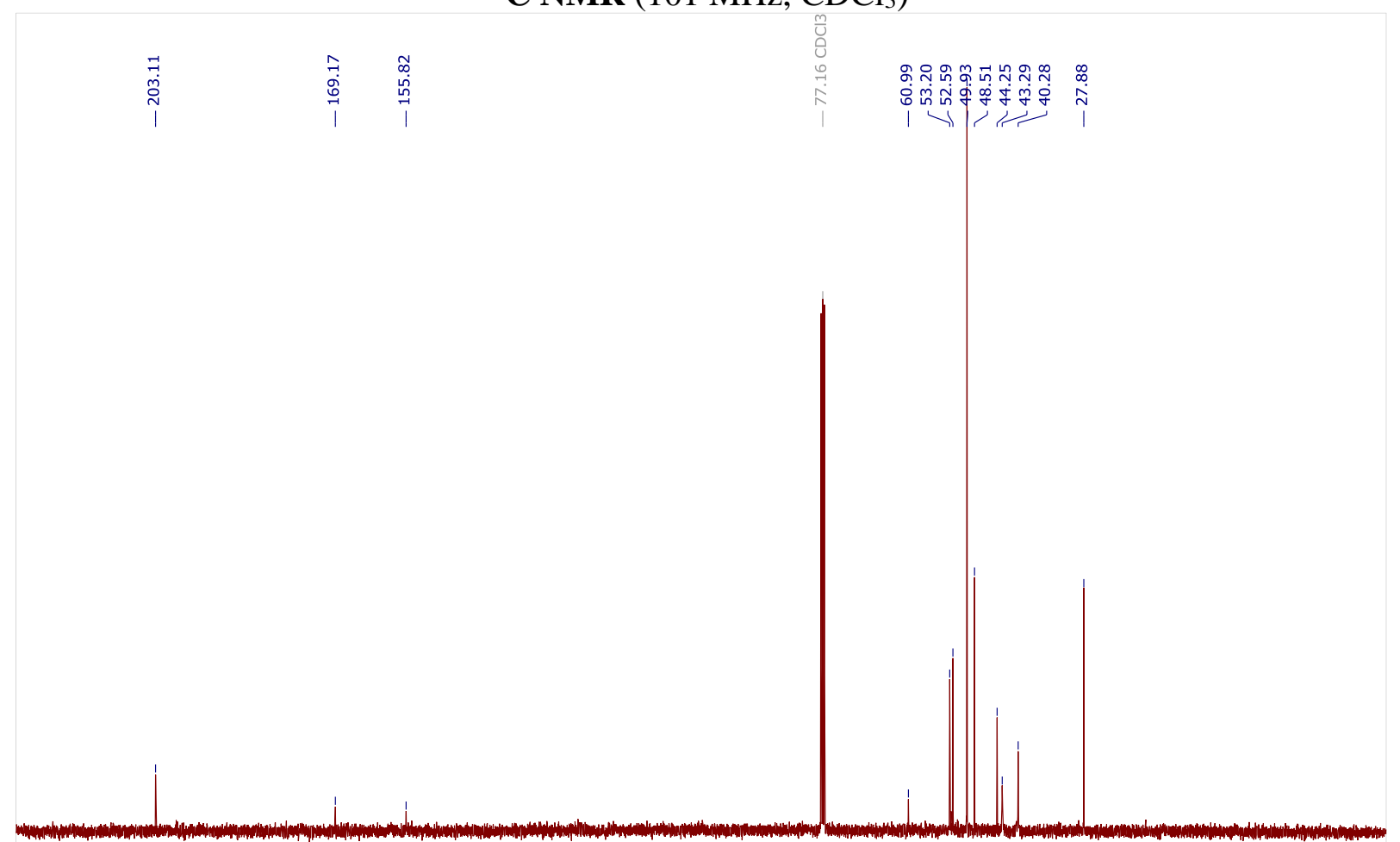

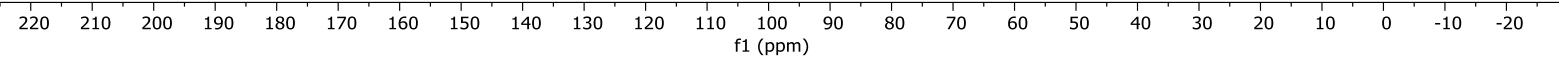


Methyl 2-(bicyclo[1.1.1]pentan-1-yl)-1-oxo-1,2,3,4-tetrahydronaphthalene-2-carboxylate, 3i

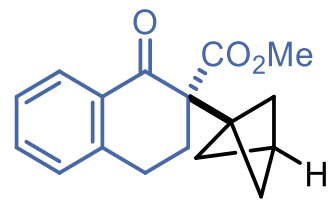

${ }^{1} \mathbf{H}$ NMR (400 MHz, $\left.\mathrm{CDCl}_{3}\right)$

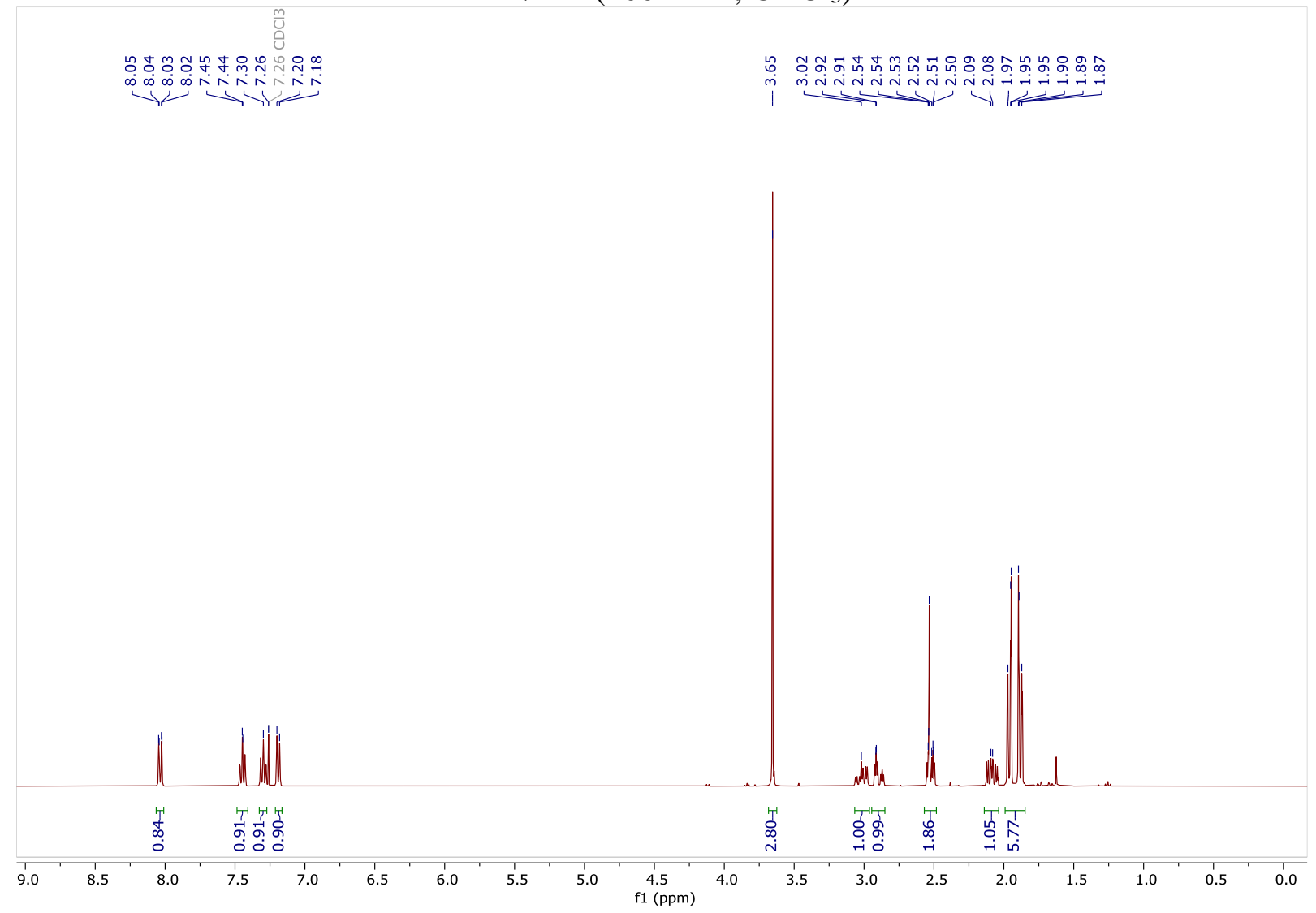

${ }^{13}$ C NMR (101 MHz, $\left.\mathrm{CDCl}_{3}\right)$

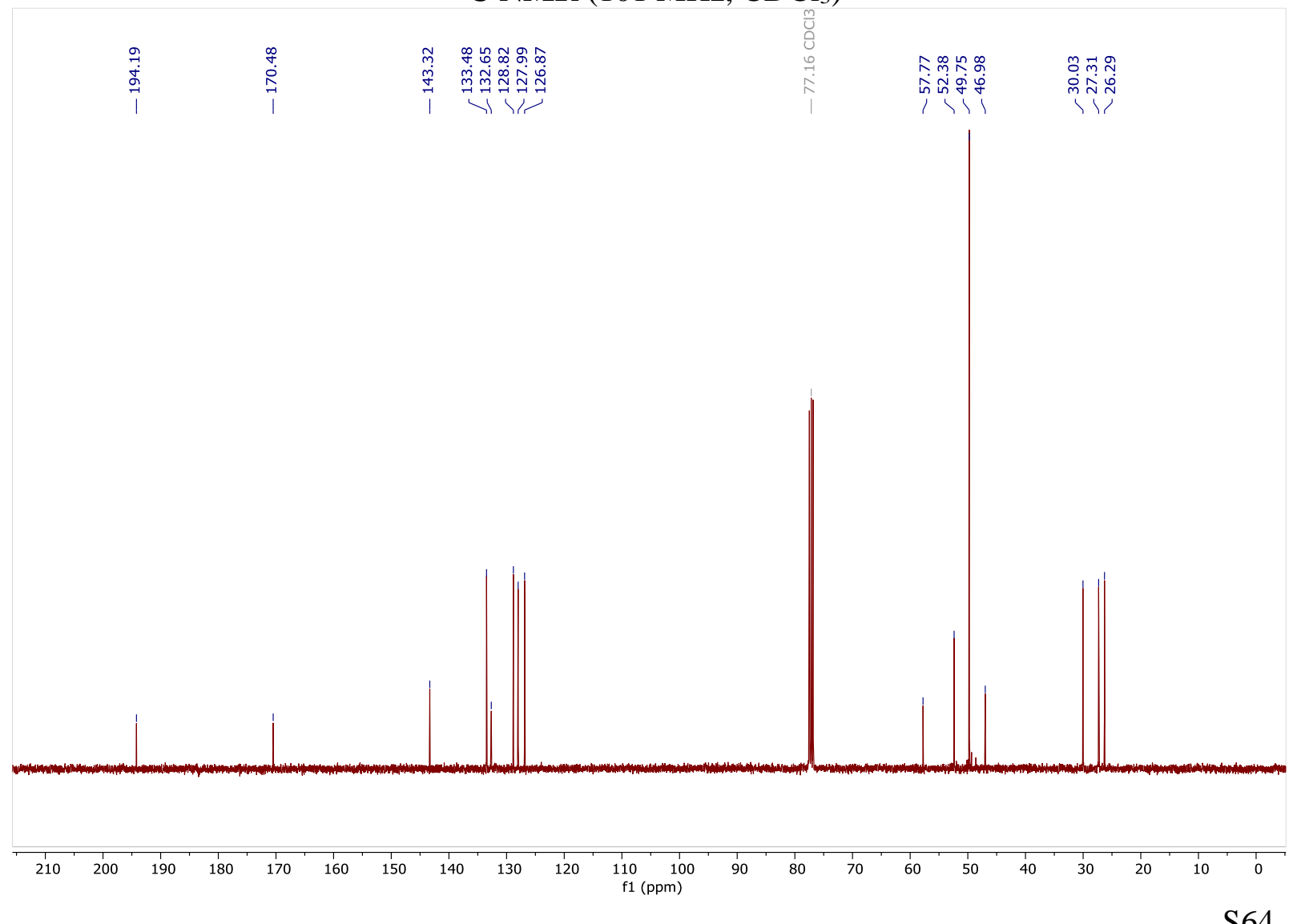


Ethyl 2-benzyl-2-(bicyclo[1.1.1]pentan-1-yl)-3-oxobutanoate, 3j<smiles></smiles>

${ }^{1} \mathbf{H}$ NMR $\left(400 \mathrm{MHz}, \mathrm{CDCl}_{3}\right)$

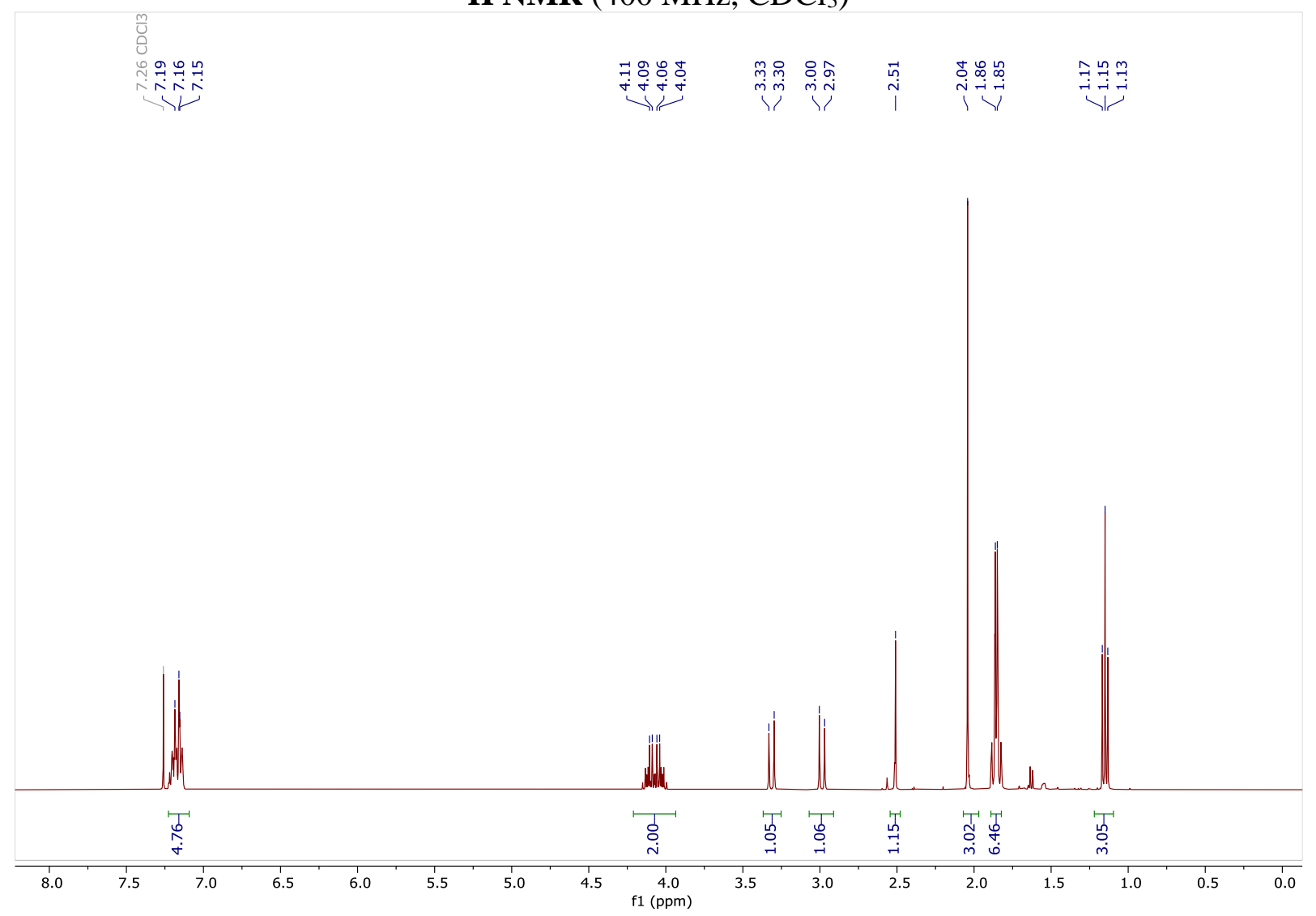

${ }^{13} \mathbf{C}$ NMR (101 MHz, $\left.\mathrm{CDCl}_{3}\right)$

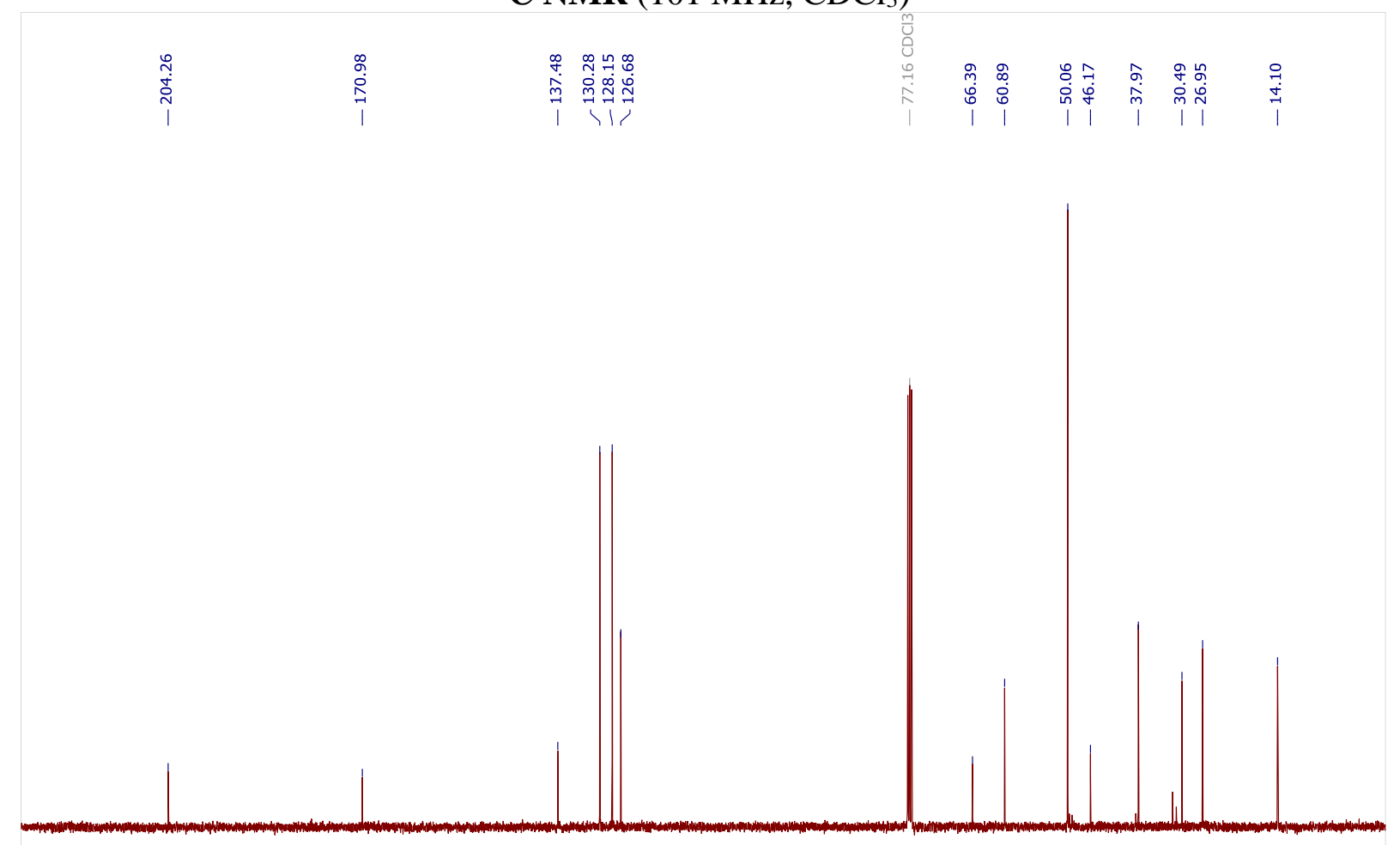

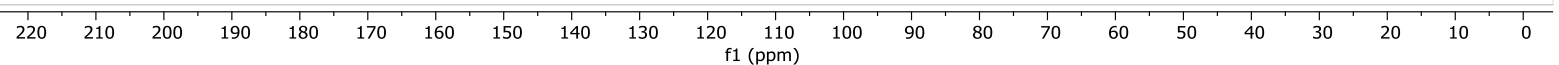


Ethyl 2-(bicyclo[1.1.1]pentan-1-yl)-3-oxo-3-phenylpropanoate, 3k

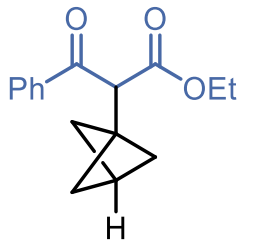

${ }^{\mathbf{1}} \mathbf{H}$ NMR (400 MHz, $\left.\mathrm{CDCl}_{3}\right)$

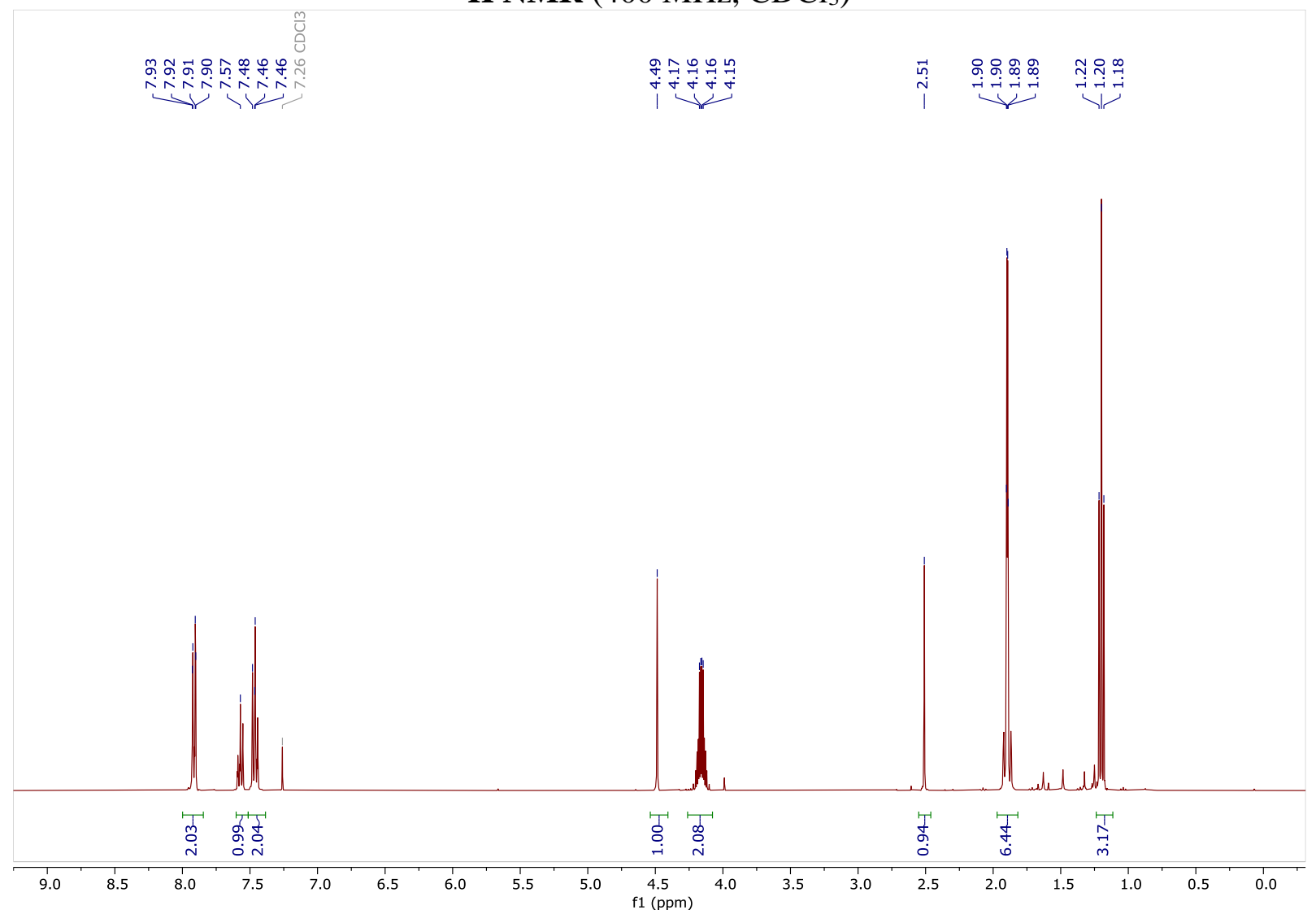

${ }^{13} \mathbf{C}$ NMR $\left(101 \mathrm{MHz}, \mathrm{CDCl}_{3}\right)$

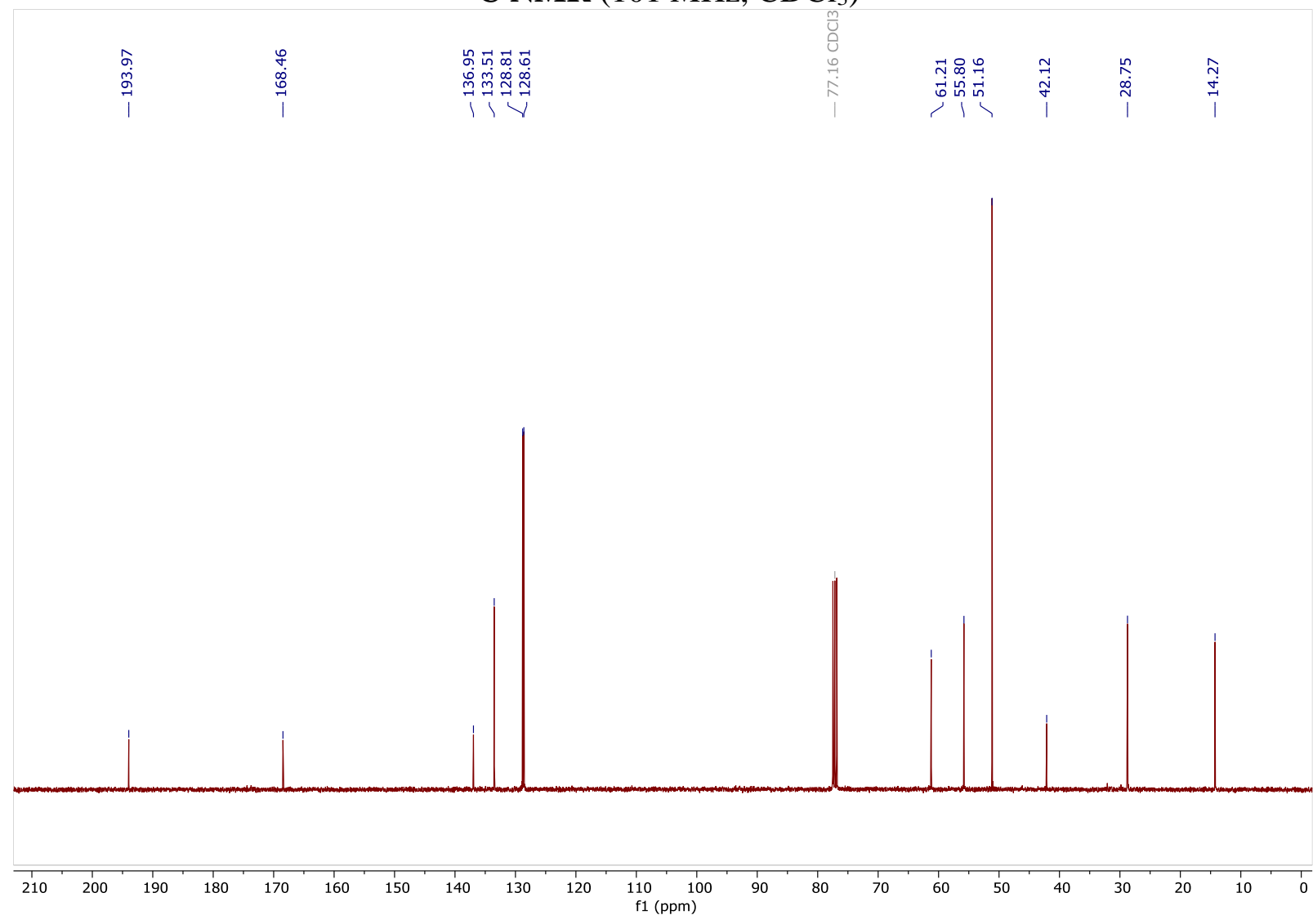



carboxylate, 31

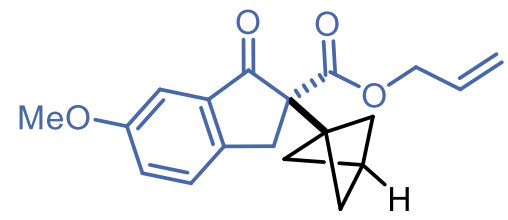

${ }^{1} \mathbf{H}$ NMR $\left(400 \mathrm{MHz}, \mathrm{CDCl}_{3}\right)$

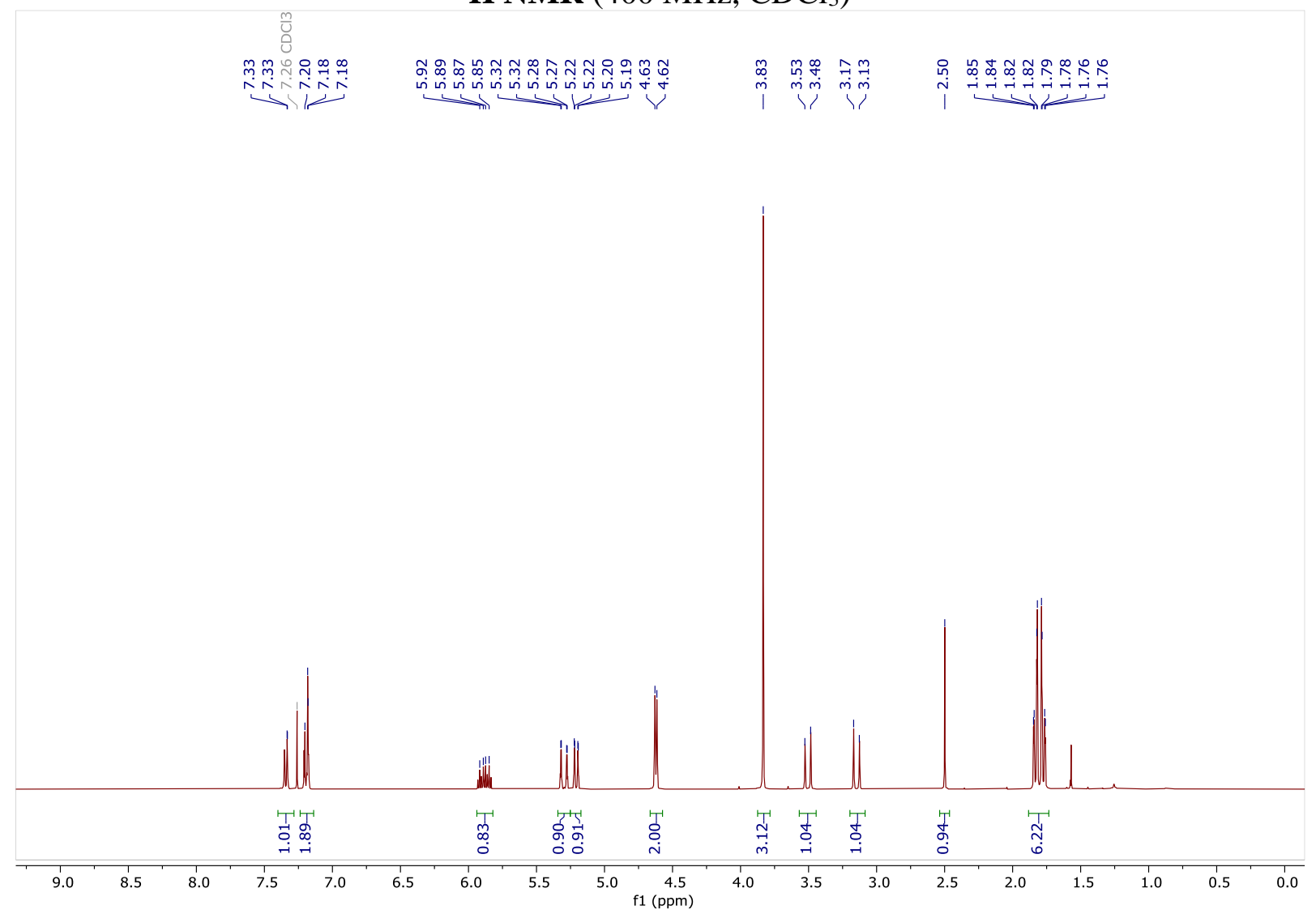

${ }^{13} \mathbf{C}$ NMR (101 MHz, $\left.\mathrm{CDCl}_{3}\right)$

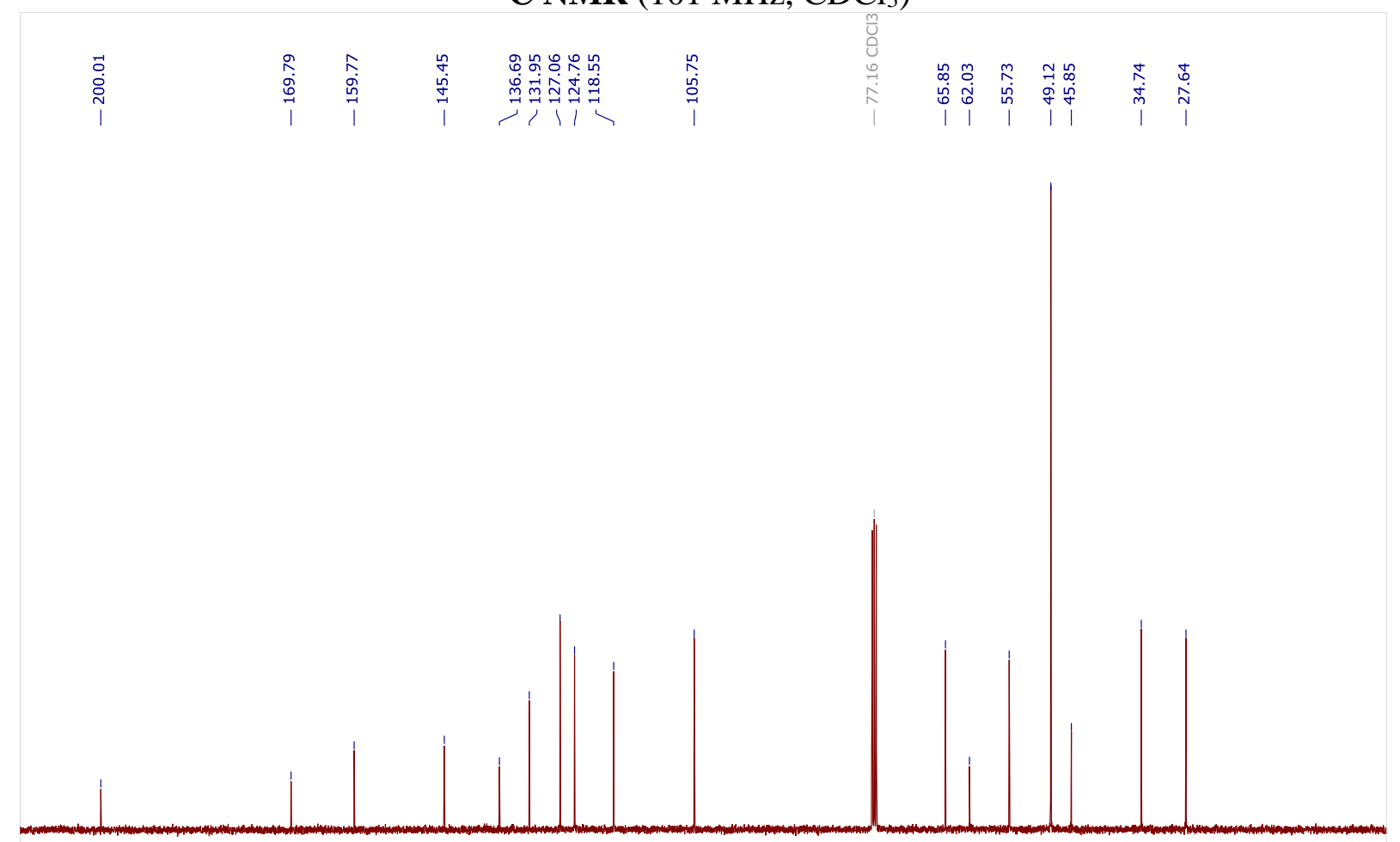

$\begin{array}{lllllllllll}210 & 200 & 190 & 180 & 170 & 160 & 150 & 140 & 130 & 120 & 110 \quad \begin{array}{l}100 \\ \text { f1 (ppm) }\end{array}\end{array}$ 
3-Allyl 1-benzyl 3-(bicyclo[1.1.1]pentan-1-yl)-4-oxopiperidine-1,3-dicarboxylate, 3m

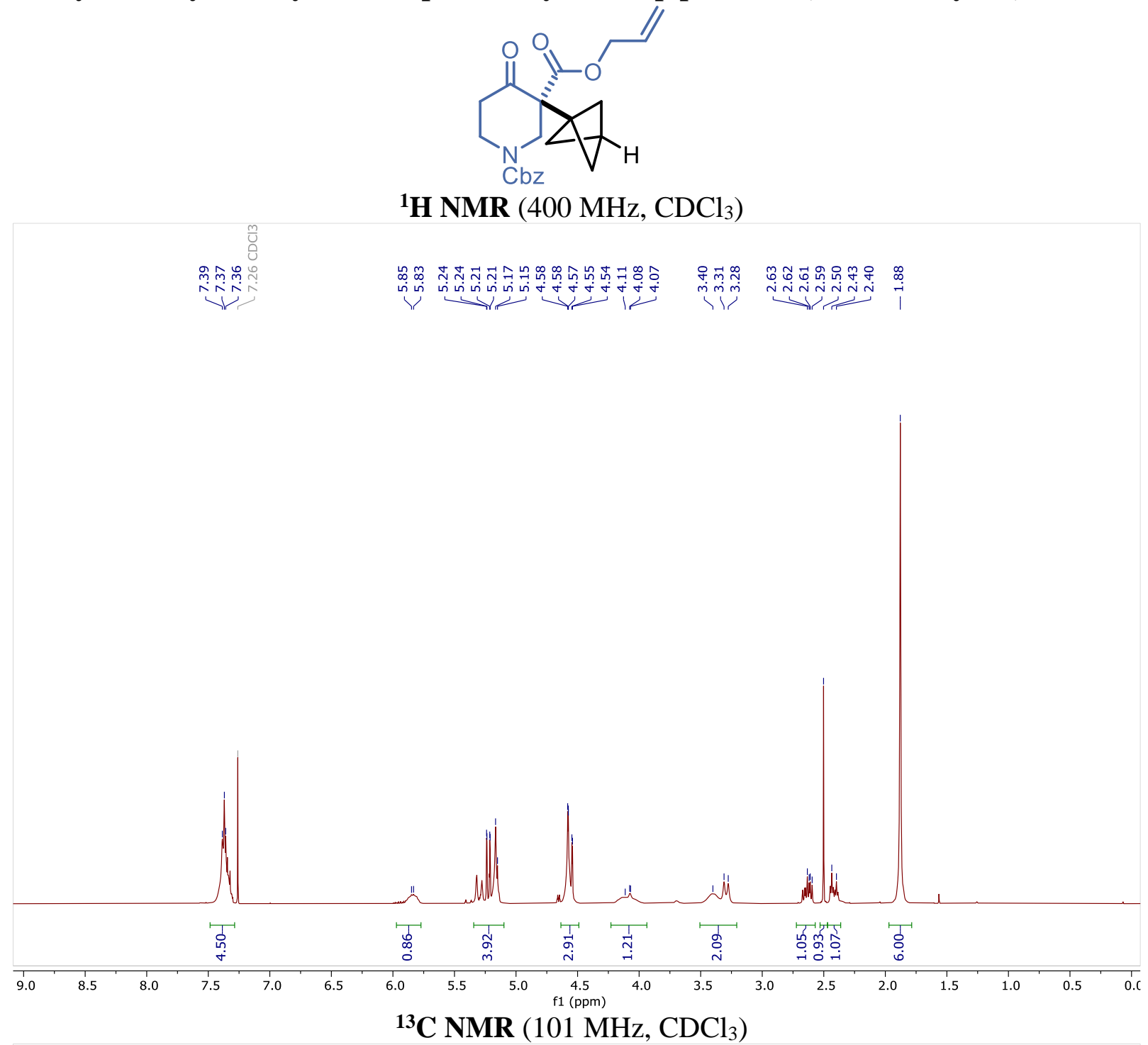

焉

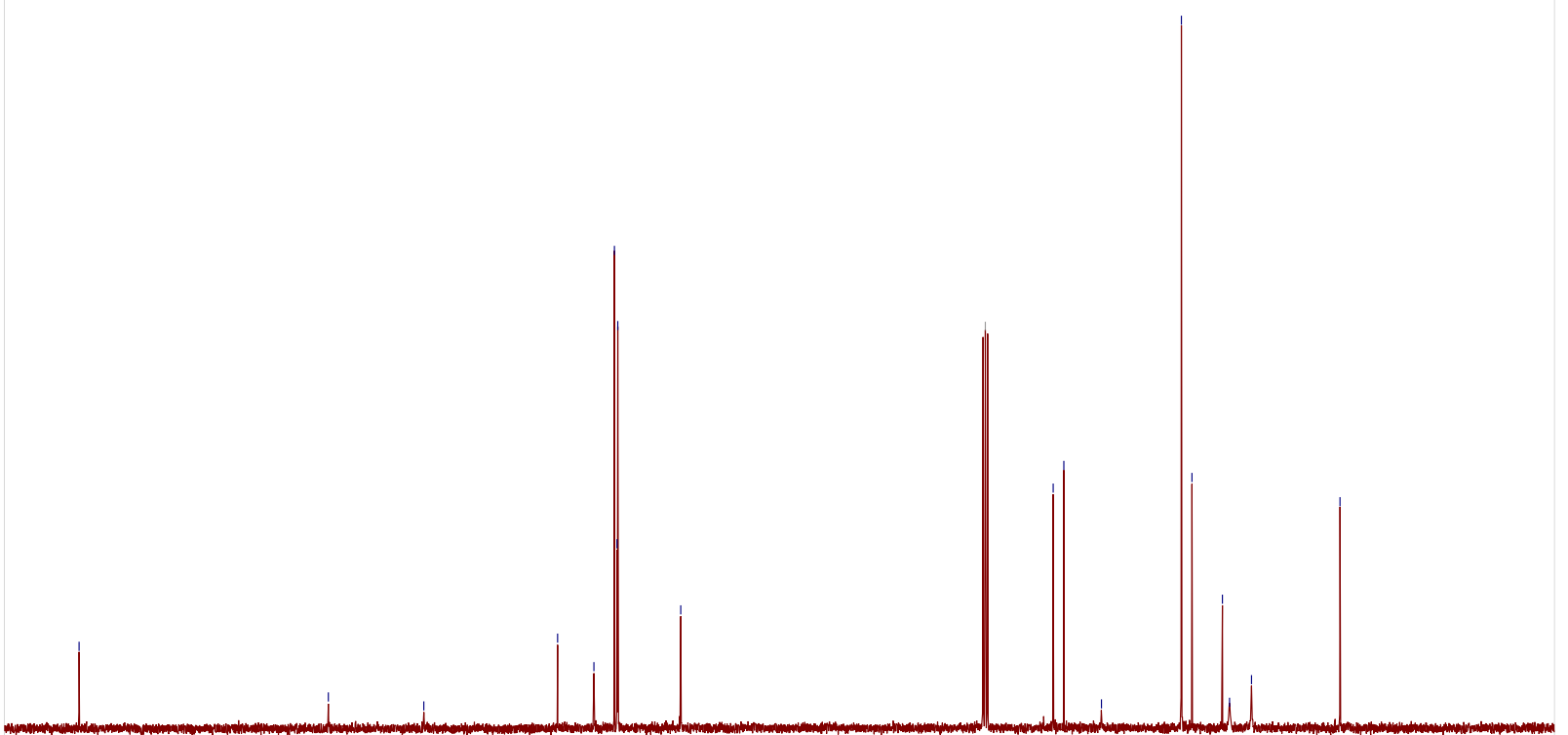

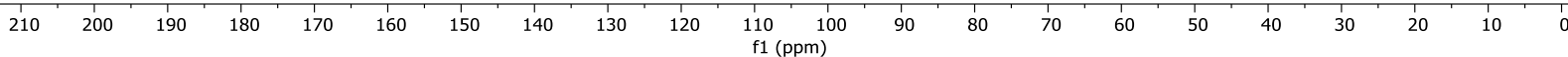


Allyl 3-(bicyclo[1.1.1]pentan-1-yl)-4-oxo-1-tosylpiperidine-3-carboxylate, 3n

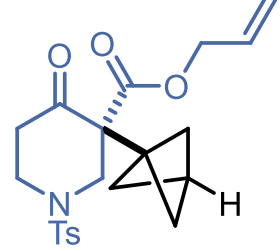

${ }^{\mathbf{1}} \mathbf{H} \mathbf{N M R}\left(400 \mathrm{MHz}, \mathrm{CDCl}_{3}\right)$

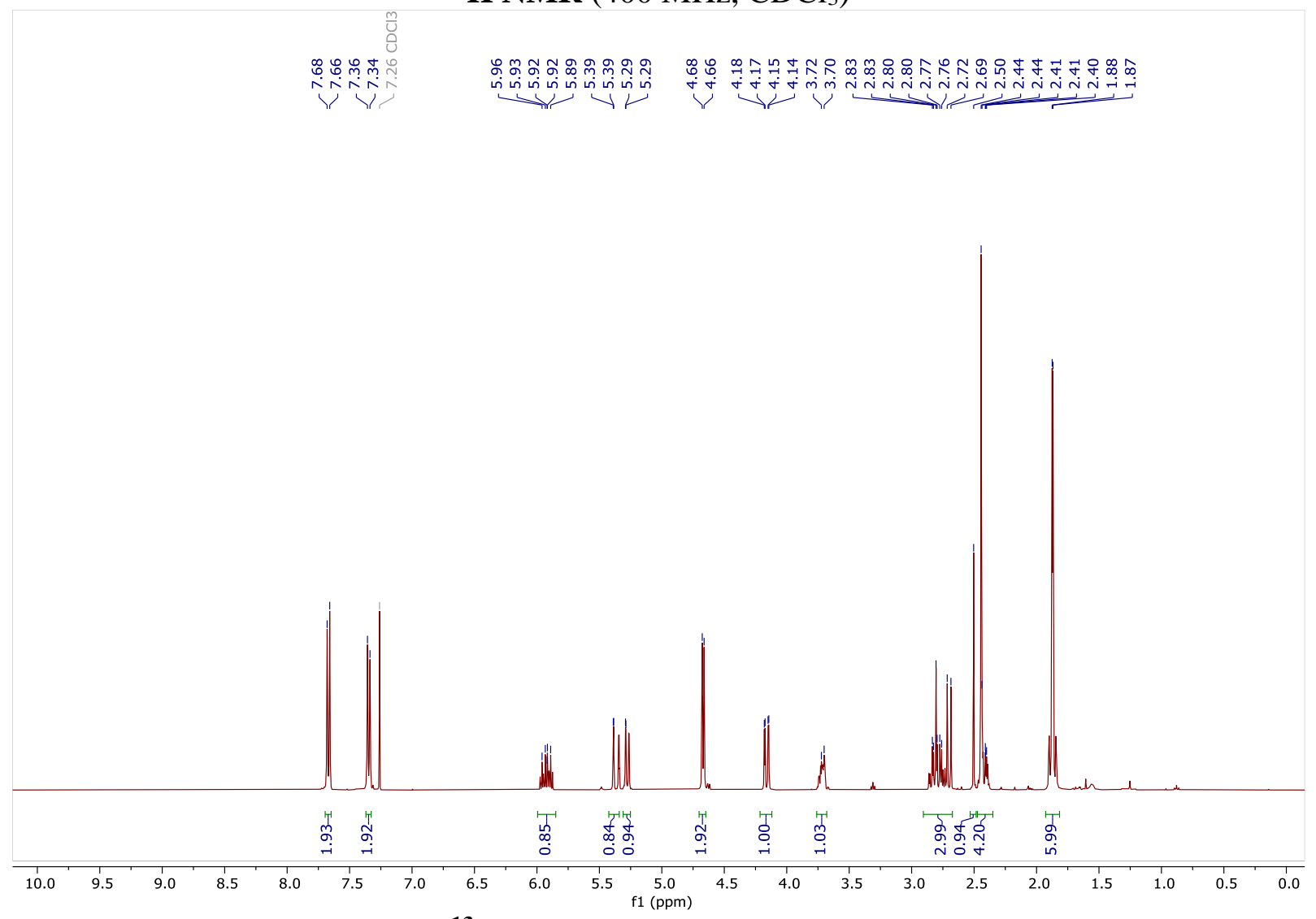

${ }^{13} \mathbf{C}$ NMR $\left(101 \mathrm{MHz}, \mathrm{CDCl}_{3}\right)$

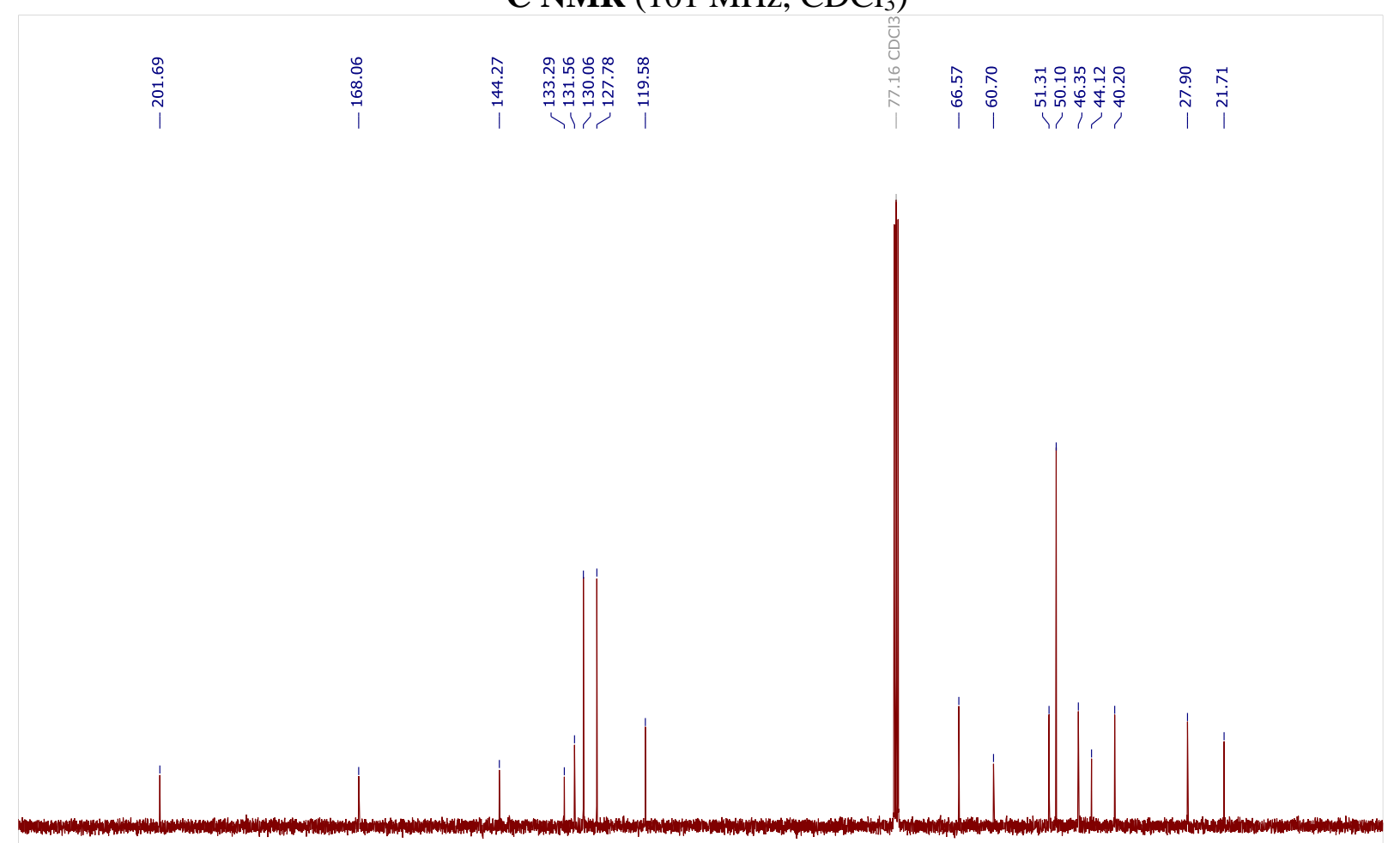

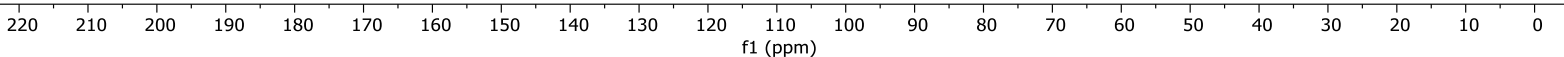


2-(Bicyclo[1.1.1]pentan-1-yl)-1-morpholinobutane-1,3-dione, 30

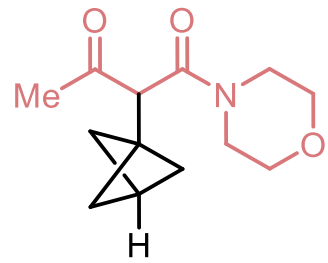

${ }^{\mathbf{1}} \mathbf{H}$ NMR $\left(400 \mathrm{MHz}, \mathrm{CDCl}_{3}\right)$

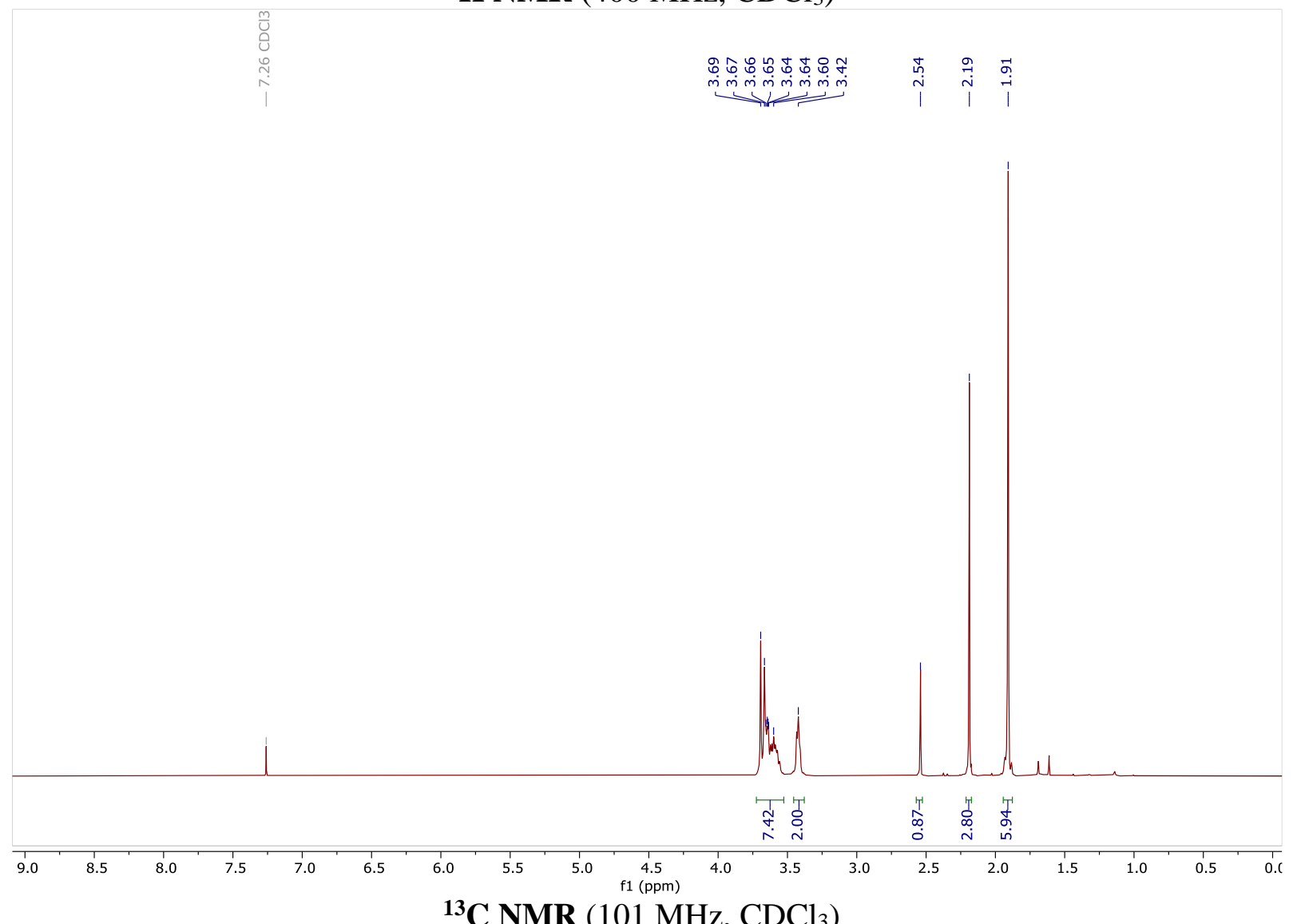

${ }^{13} \mathbf{C}$ NMR $\left(101 \mathrm{MHz}, \mathrm{CDCl}_{3}\right)$

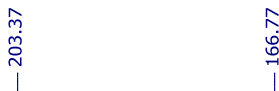

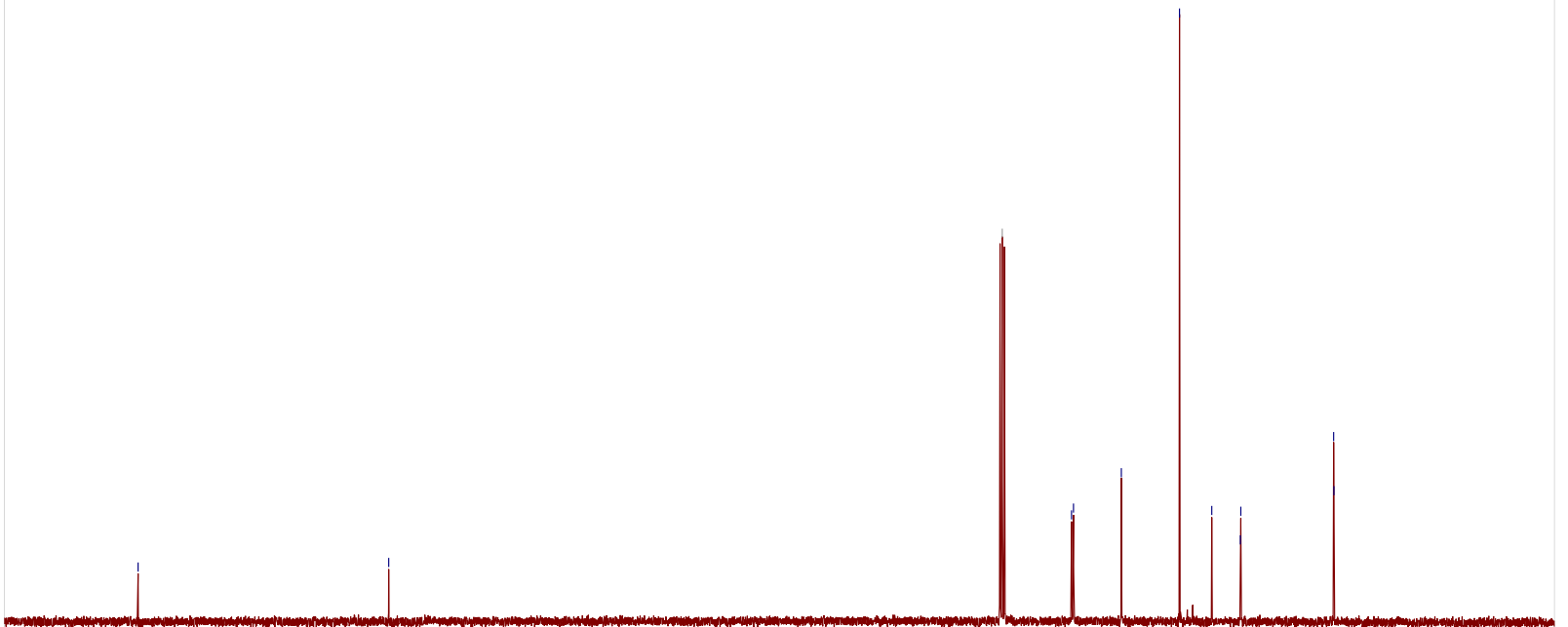


2-(Bicyclo[1.1.1]pentan-1-yl)-3-oxo- $N$-(thiazol-2-yl)butanamide, 3p

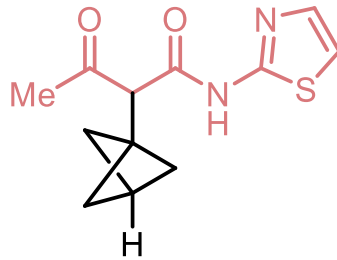

${ }^{\mathbf{1}} \mathbf{H}$ NMR (400 MHz, $\left.\mathrm{CDCl}_{3}\right)$

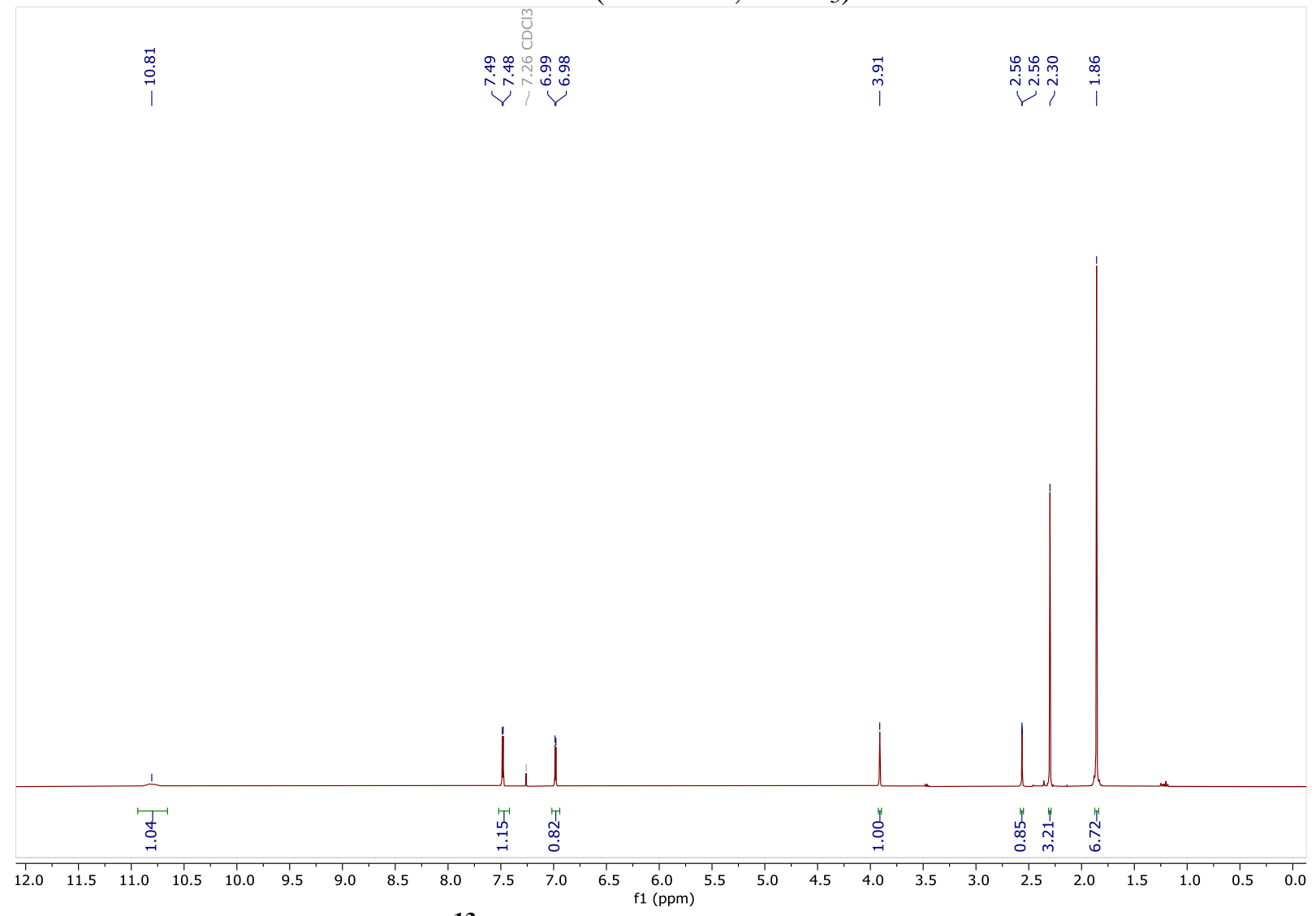

${ }^{13}$ C NMR (101 MHz, $\left.\mathrm{CDCl}_{3}\right)$

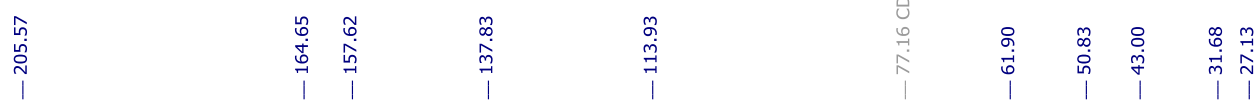

\begin{tabular}{l|llllllllllllllllllllll}
1 & 1 \\
220 & 210 & 200 & 190 & 180 & 170 & 160 & 150 & 140 & 130 & 120 & 110 & 100 & 90 & 80 & 70 & 60 & 50 & 40 & 30 & 20 & 10 & 0
\end{tabular} 
3-Acetyl-1-benzyl-3-(bicyclo[1.1.1]pentan-1-yl)pyrrolidin-2-one, 3q

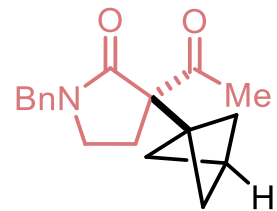

${ }^{1} \mathbf{H}$ NMR $\left(400 \mathrm{MHz}, \mathrm{CDCl}_{3}\right)$

\begin{tabular}{|c|c|c|c|}
\hline 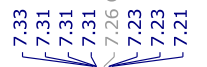 & 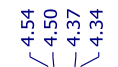 & 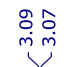 & 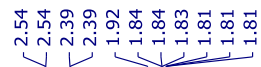 \\
\hline
\end{tabular}

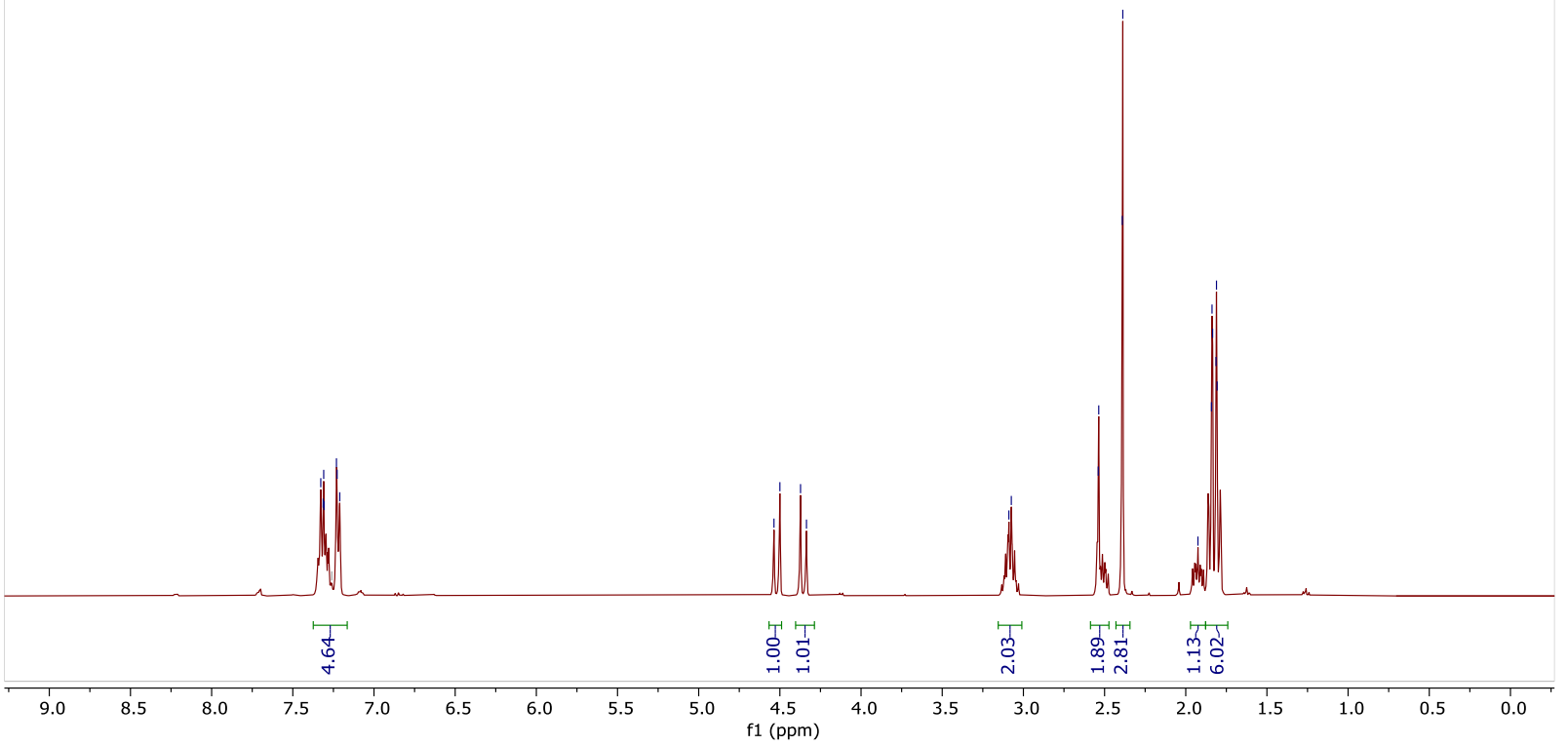

${ }^{13} \mathbf{C ~ N M R}\left(101 \mathrm{MHz}, \mathrm{CDCl}_{3}\right)$

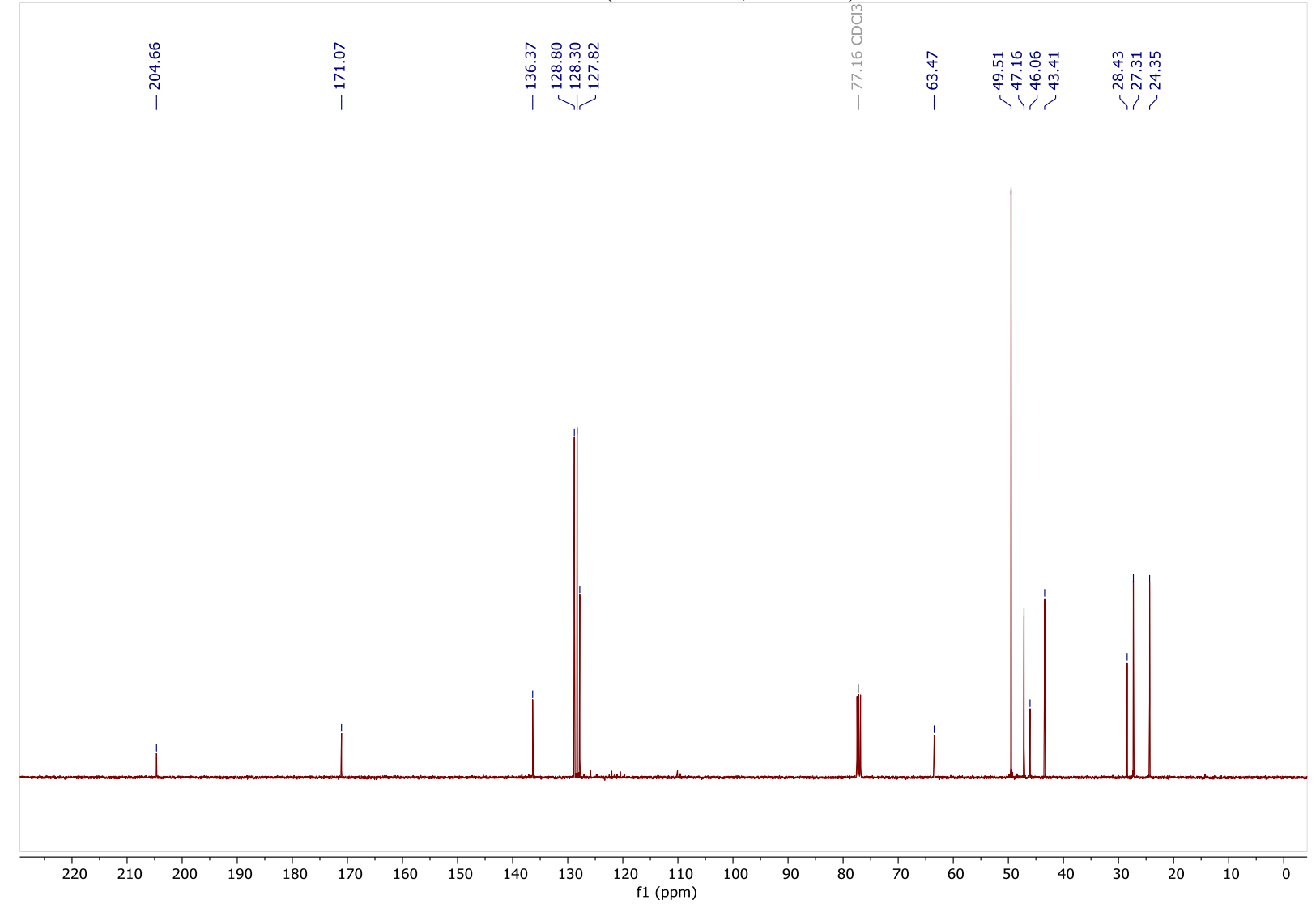


3-(bicyclo[1.1.1]pentan-1-yl)-1-methyl-3-nicotinoylpyrrolidin-2-one, 3r

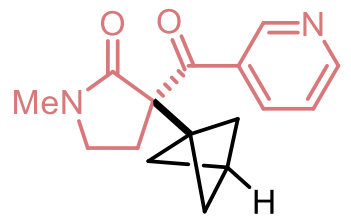

${ }^{1} \mathbf{H}$ NMR (400 MHz, $\left.\mathrm{CDCl}_{3}\right)$

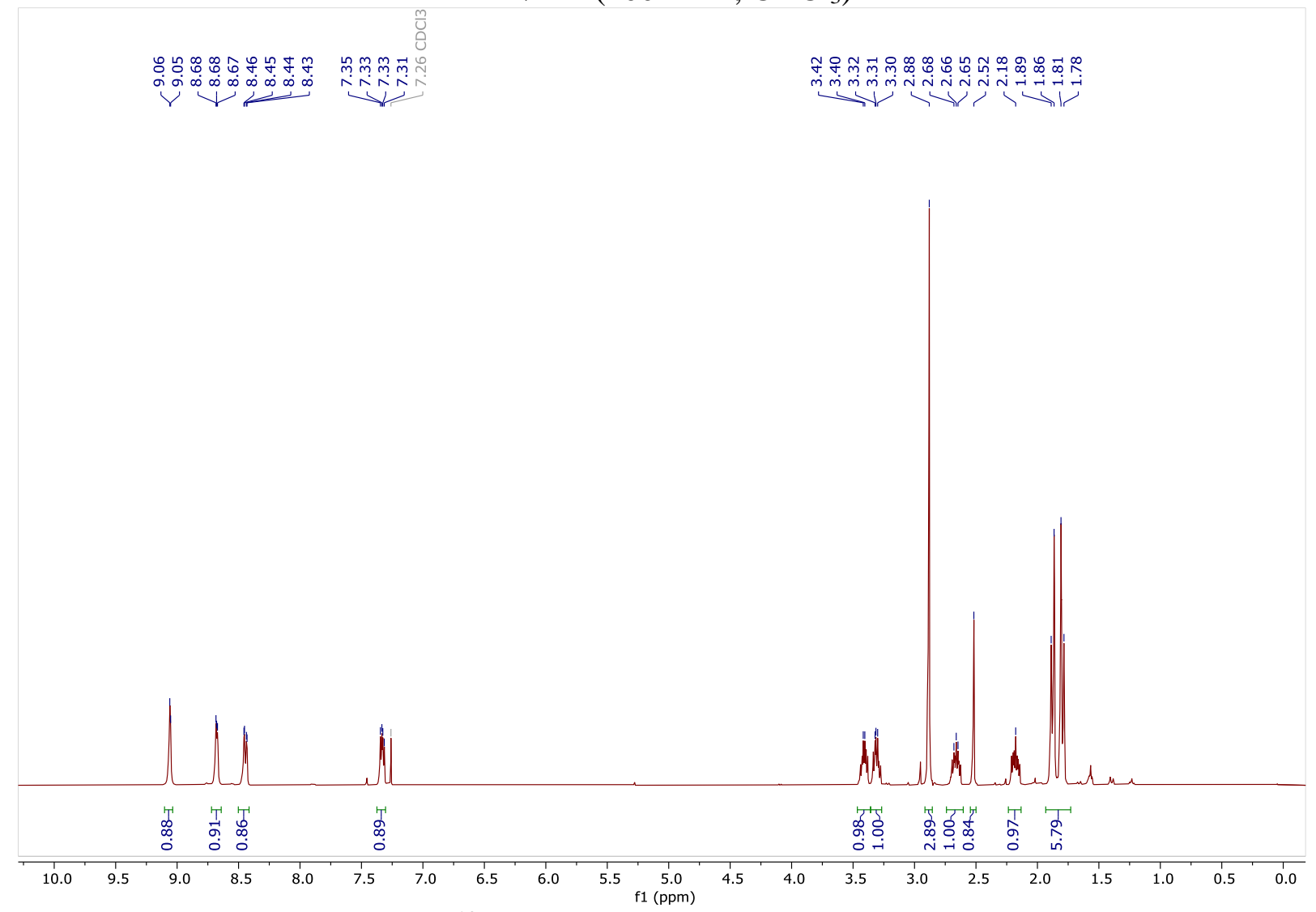

${ }^{13} \mathbf{C}$ NMR $\left(101 \mathrm{MHz}, \mathrm{CDCl}_{3}\right)$

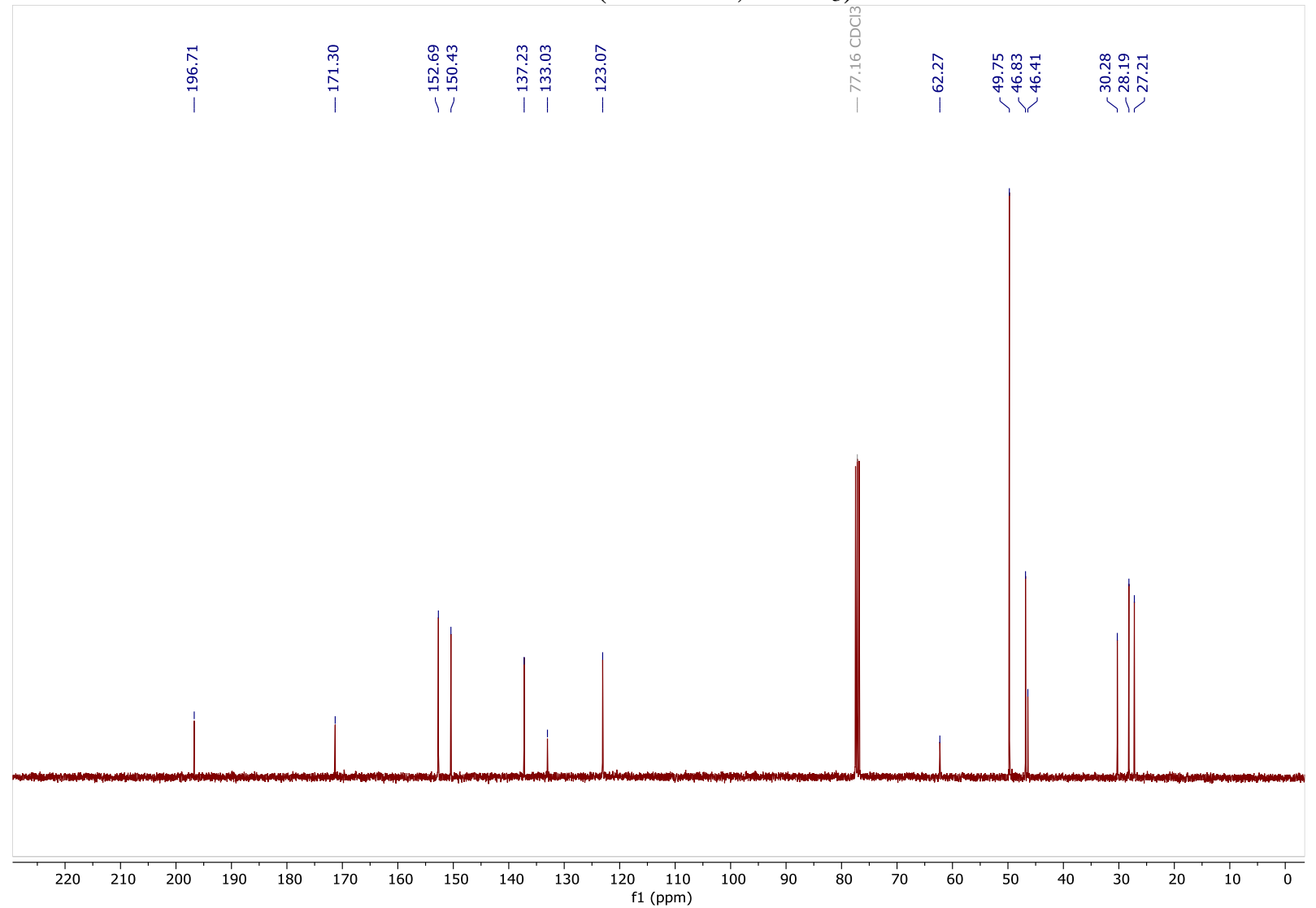


2-(Bicyclo[1.1.1]pentan-1-yl)-1,3-diphenylpropane-1,3-dione, 3s

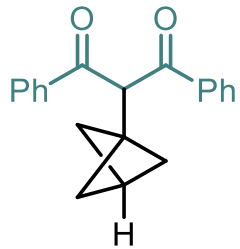

${ }^{1} \mathbf{H}$ NMR (400 MHz, $\left.\mathrm{CDCl}_{3}\right)$

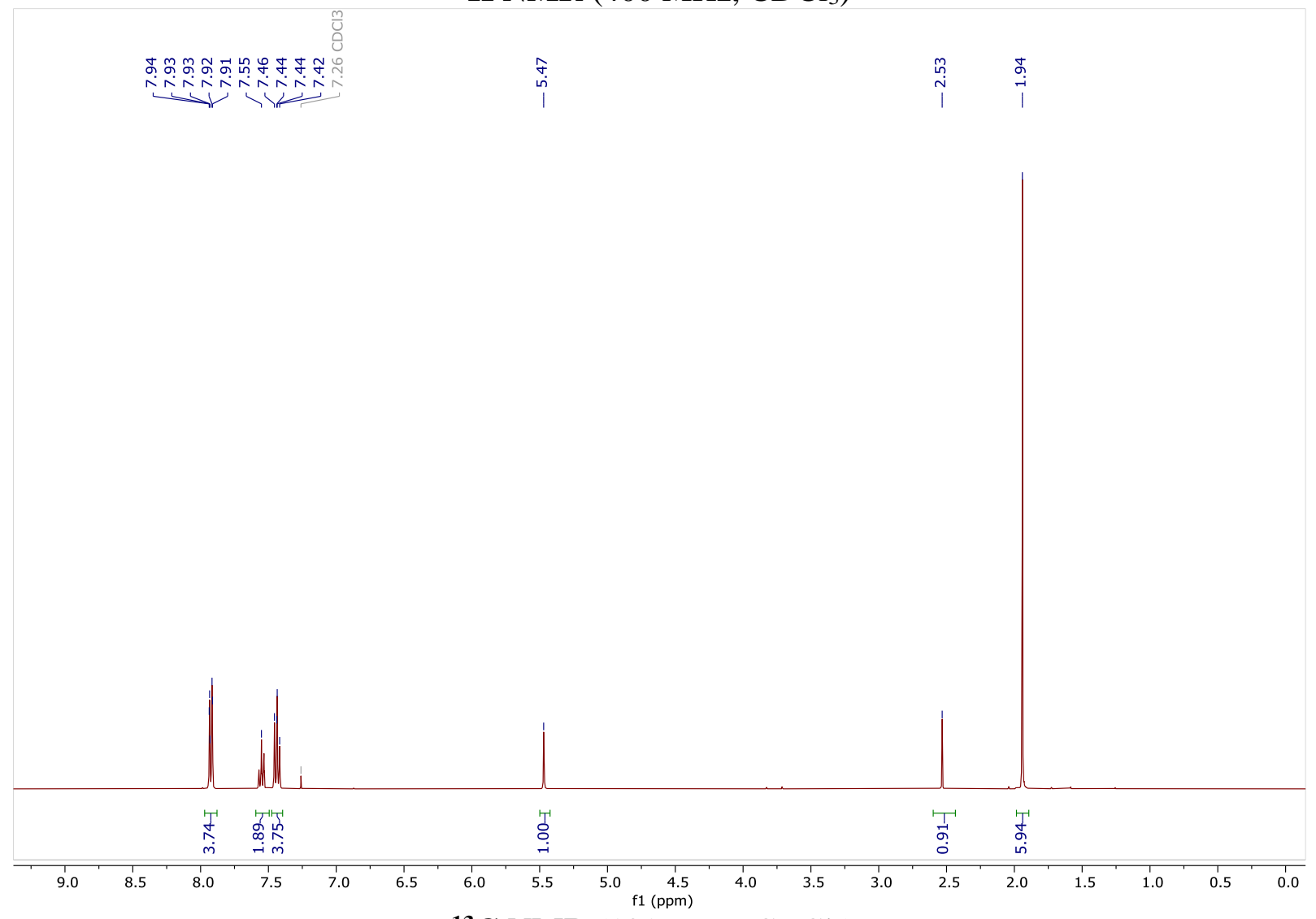

${ }^{13} \mathbf{C}$ NMR (101 MHz, $\left.\mathrm{CDCl}_{3}\right)$

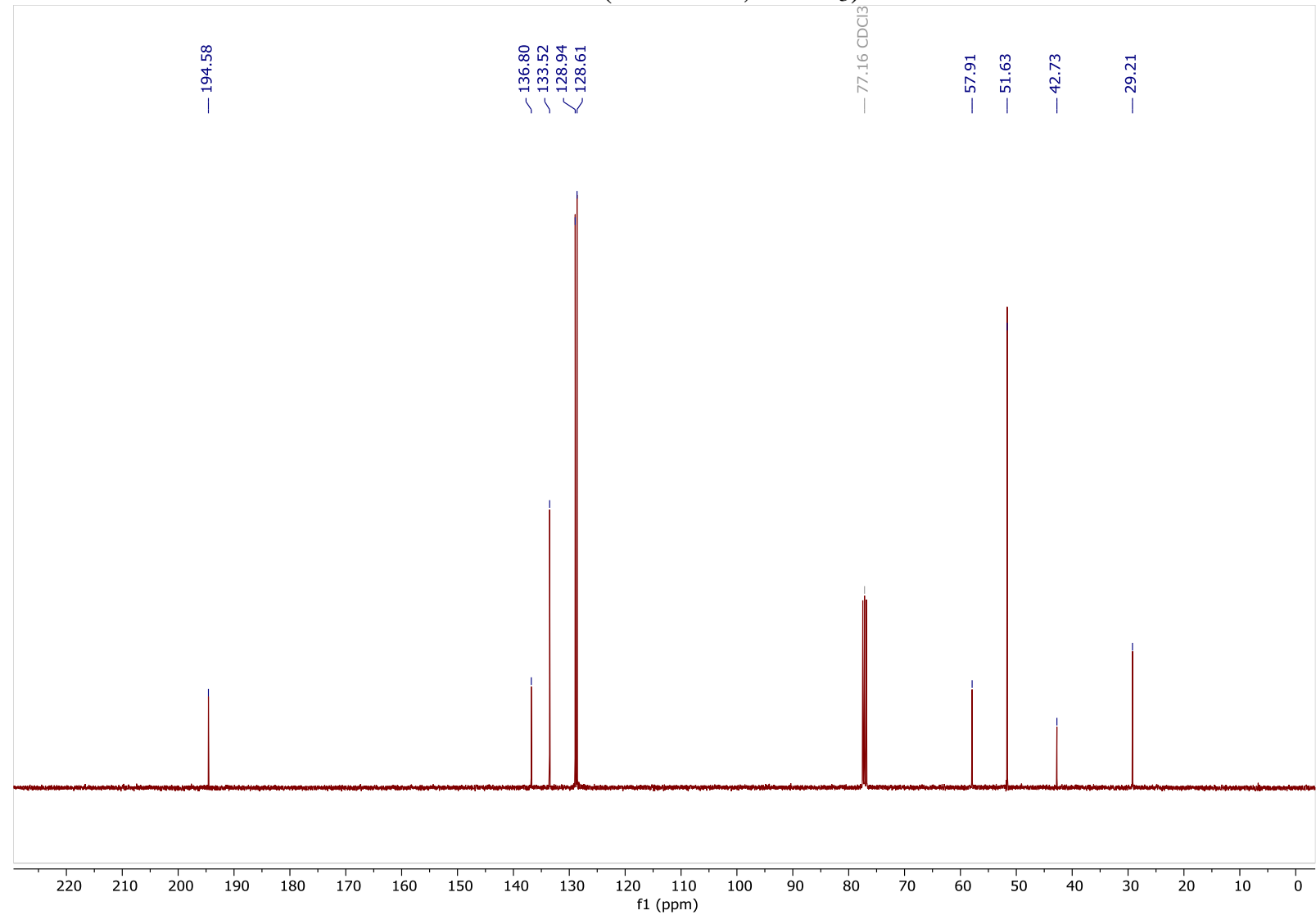


2-Acetyl-2-(bicyclo[1.1.1]pentan-1-yl)cyclopentan-1-one, 3t

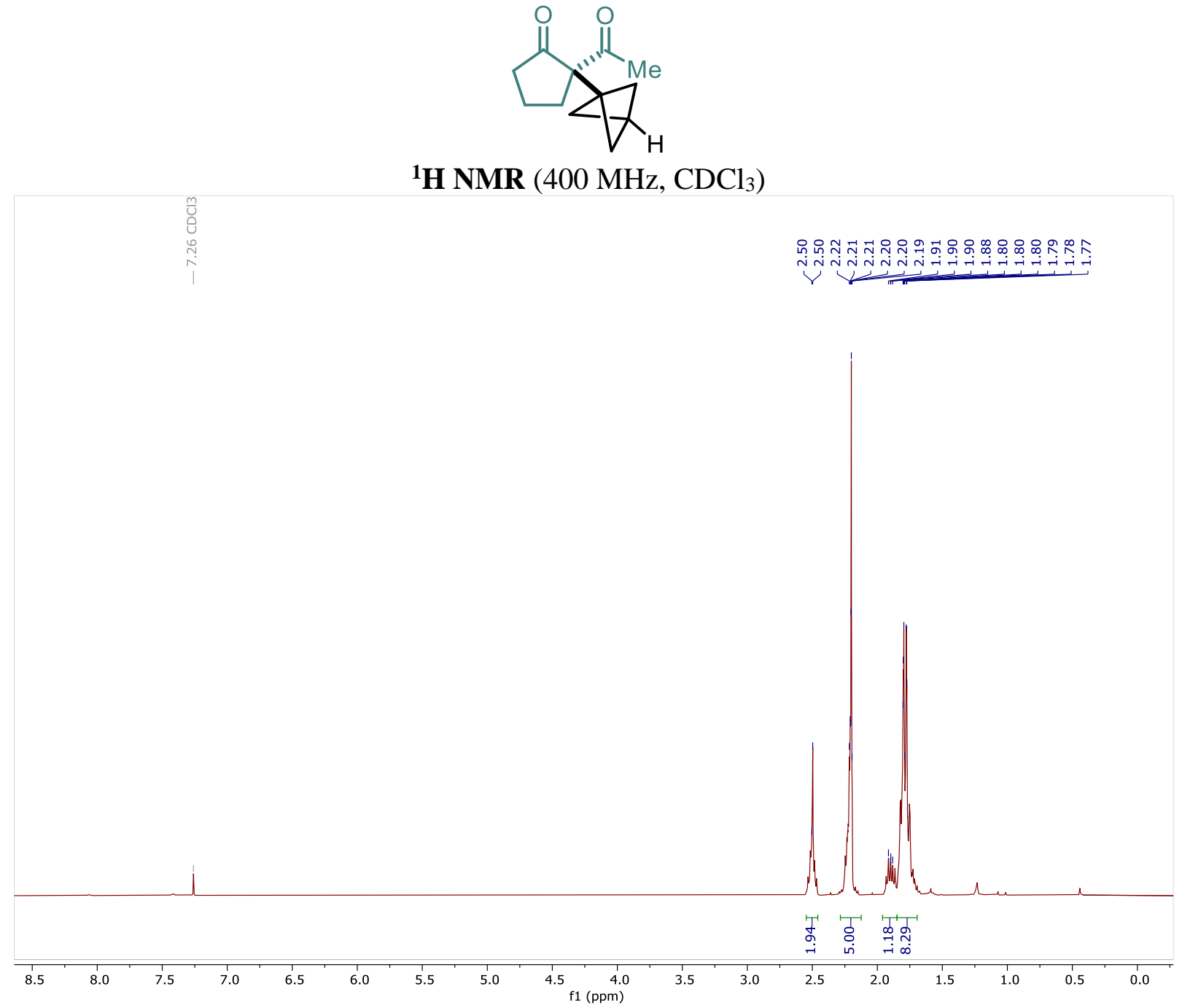

${ }^{13}$ C NMR (101 MHz, $\left.\mathrm{CDCl}_{3}\right)$

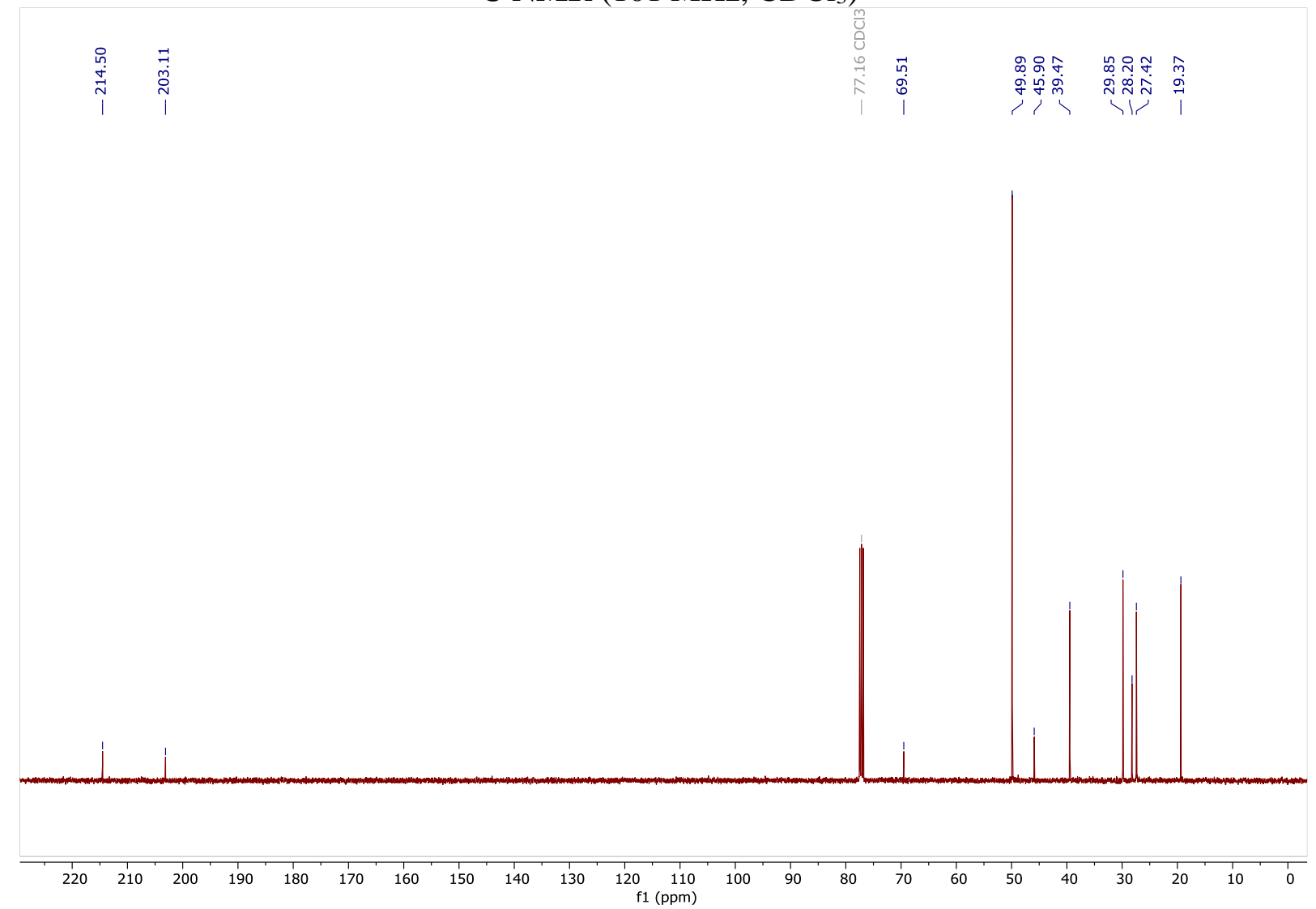


1-(Bicyclo[1.1.1]pentan-1-yl)-2-oxocyclopentane-1-carbonitrile, $3 u$

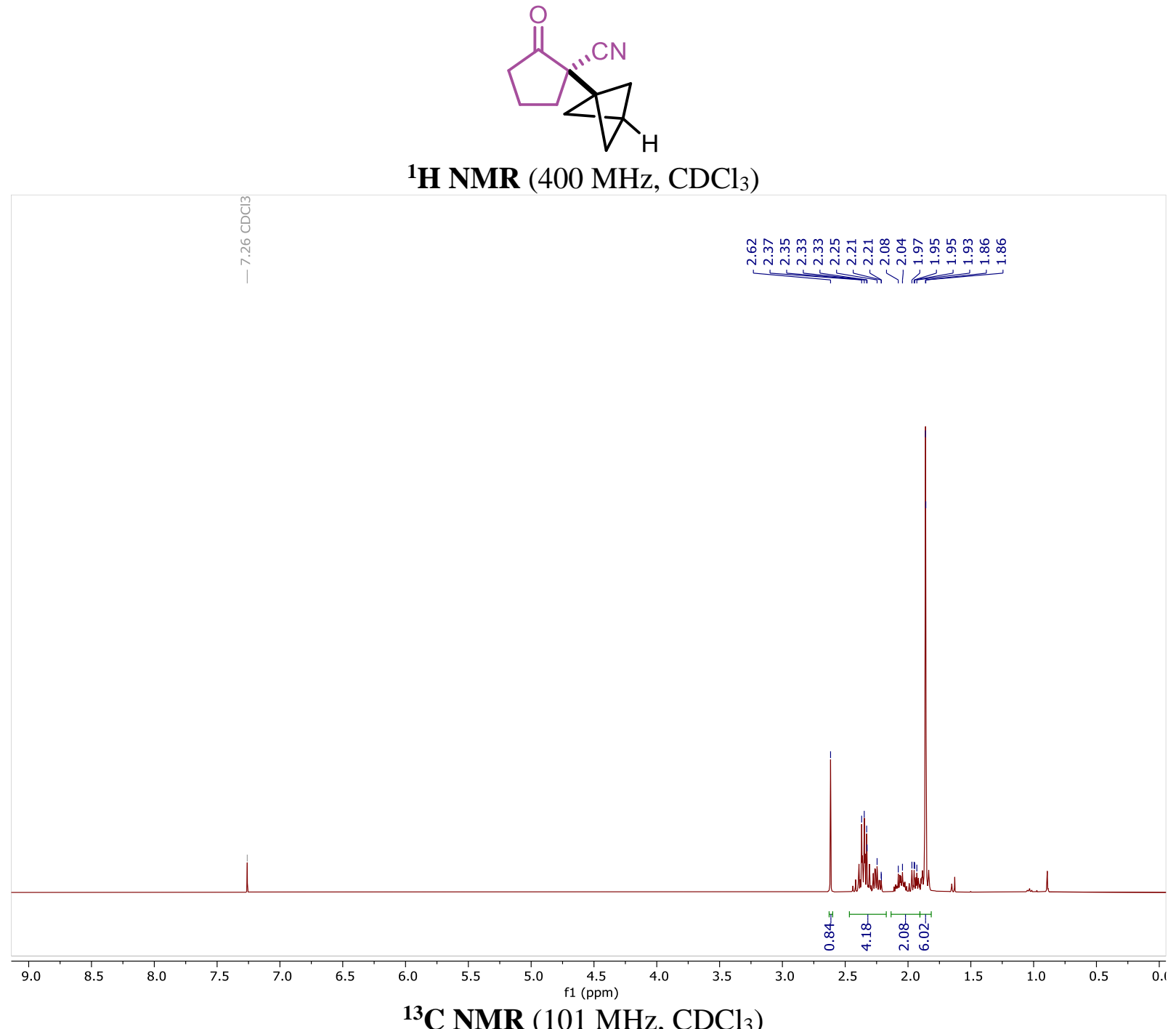

${ }^{13} \mathbf{C ~ N M R}\left(101 \mathrm{MHz}, \mathrm{CDCl}_{3}\right)$

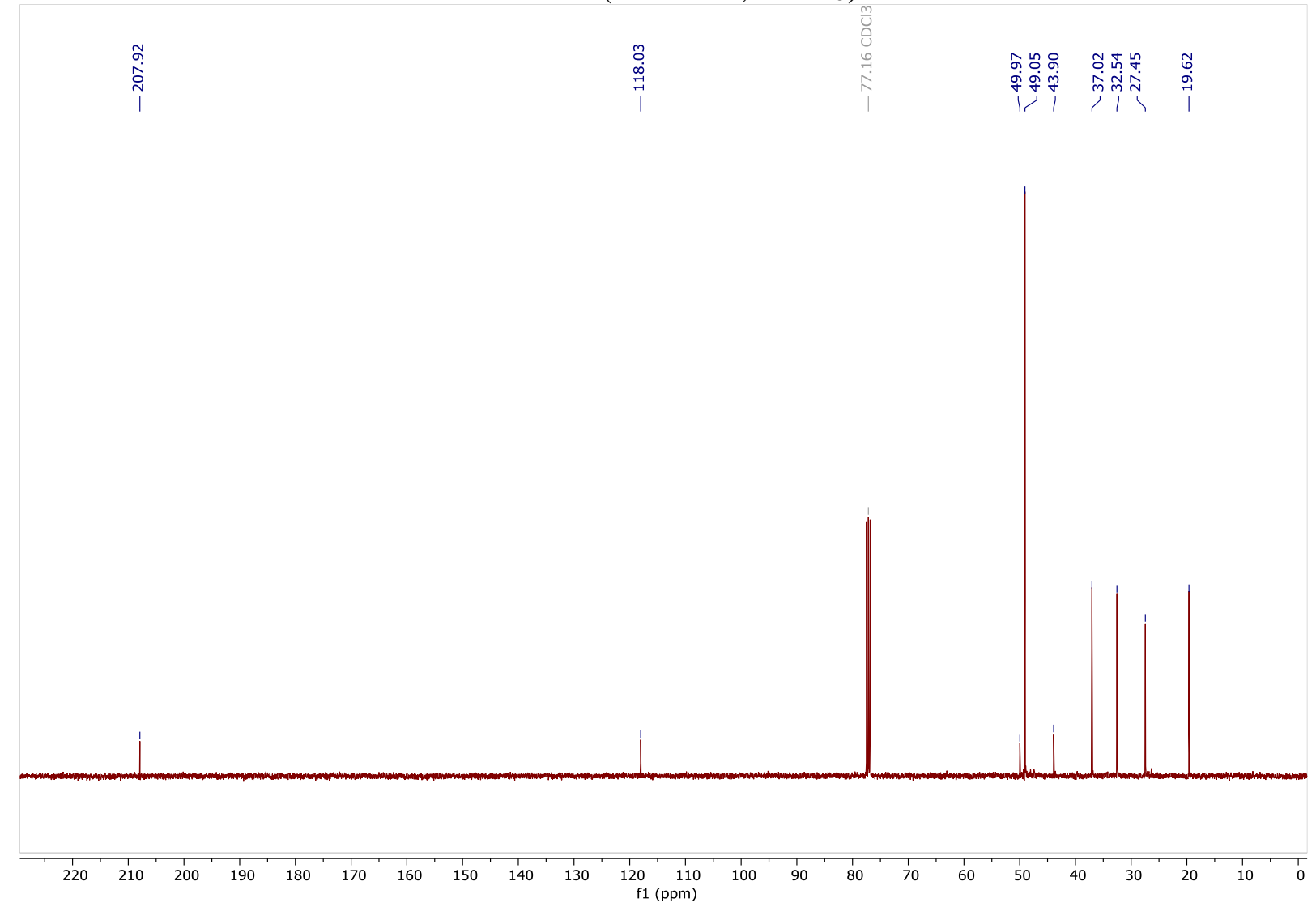


Methyl 2-(bicyclo[1.1.1]pentan-1-yl)-8-methyl-3-oxo-8-azabicyclo[3.2.1]octane-2carboxylate, $3 \mathrm{v}$

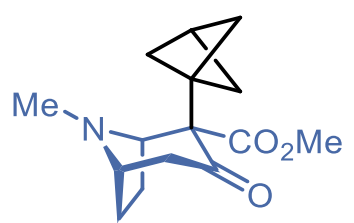

${ }^{1} \mathbf{H}$ NMR (400 MHz, $\left.\mathrm{CDCl}_{3}\right)$

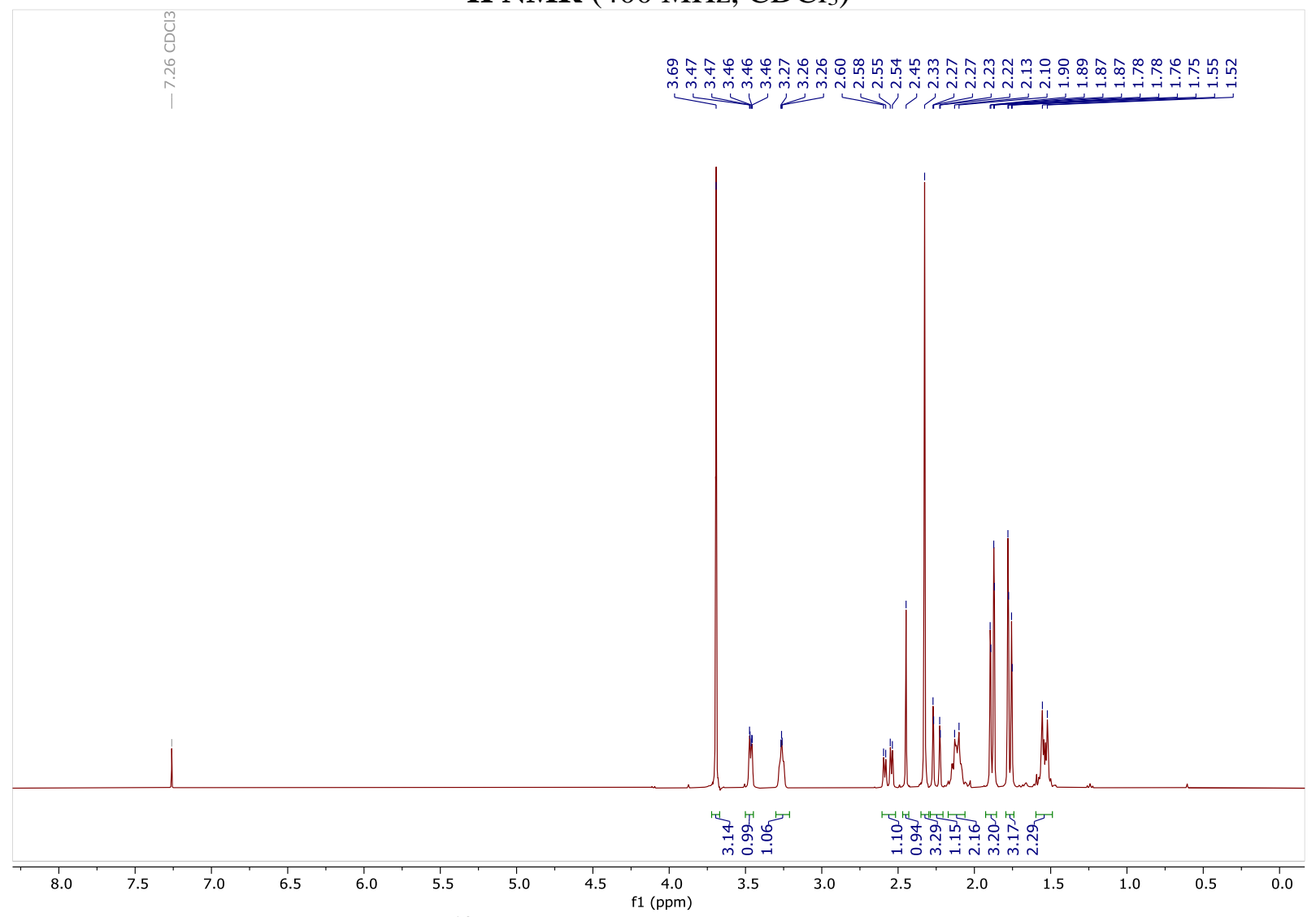

${ }^{13}$ C NMR (101 MHz, $\left.\mathrm{CDCl}_{3}\right)$

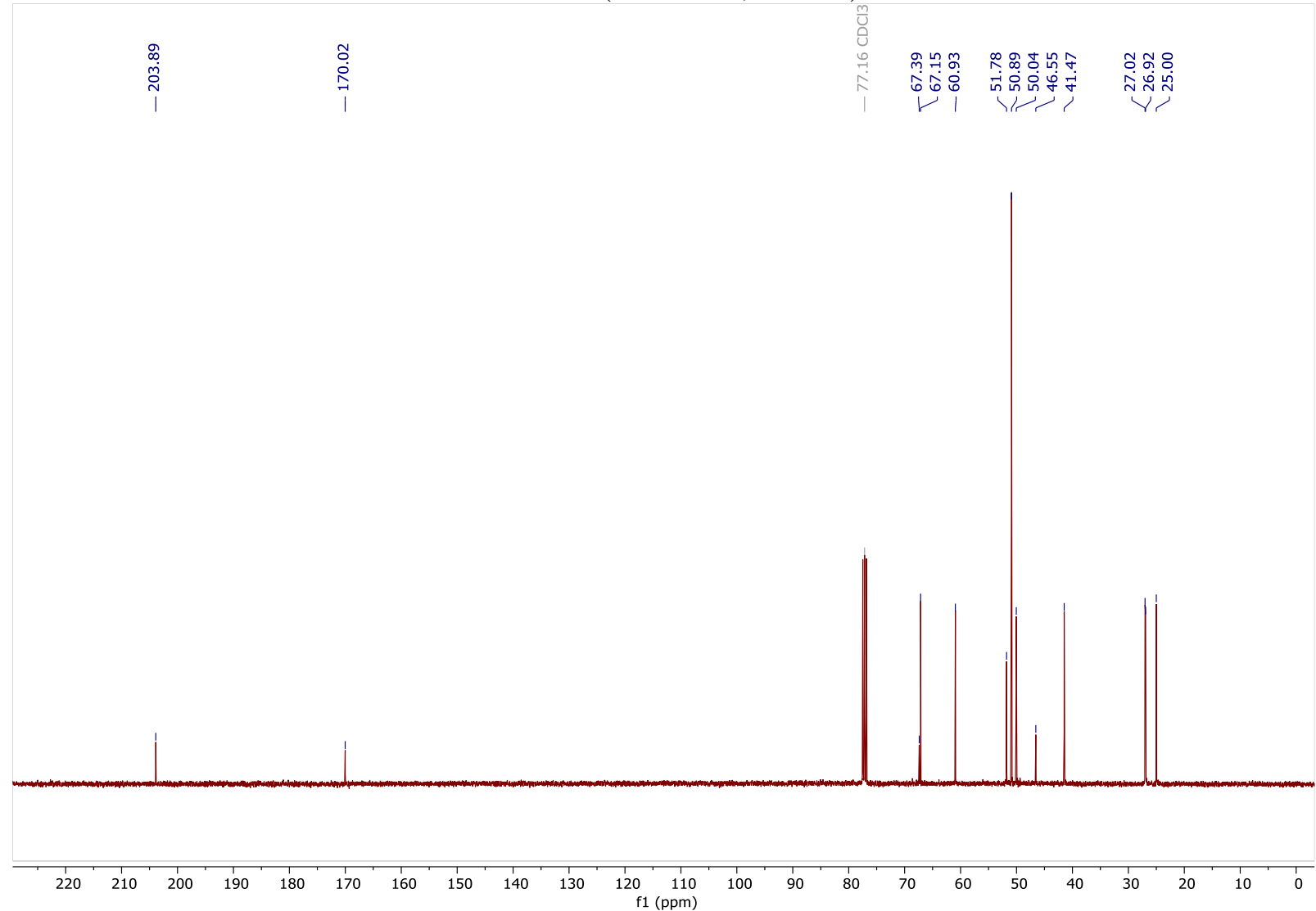


BCP-16-carboxymestrone methyl ester, 3w

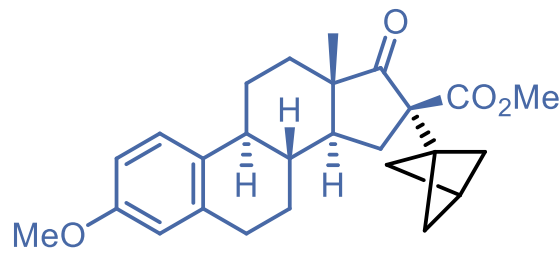

${ }^{1} \mathbf{H}$ NMR $\left(400 \mathrm{MHz}, \mathrm{CDCl}_{3}\right)$

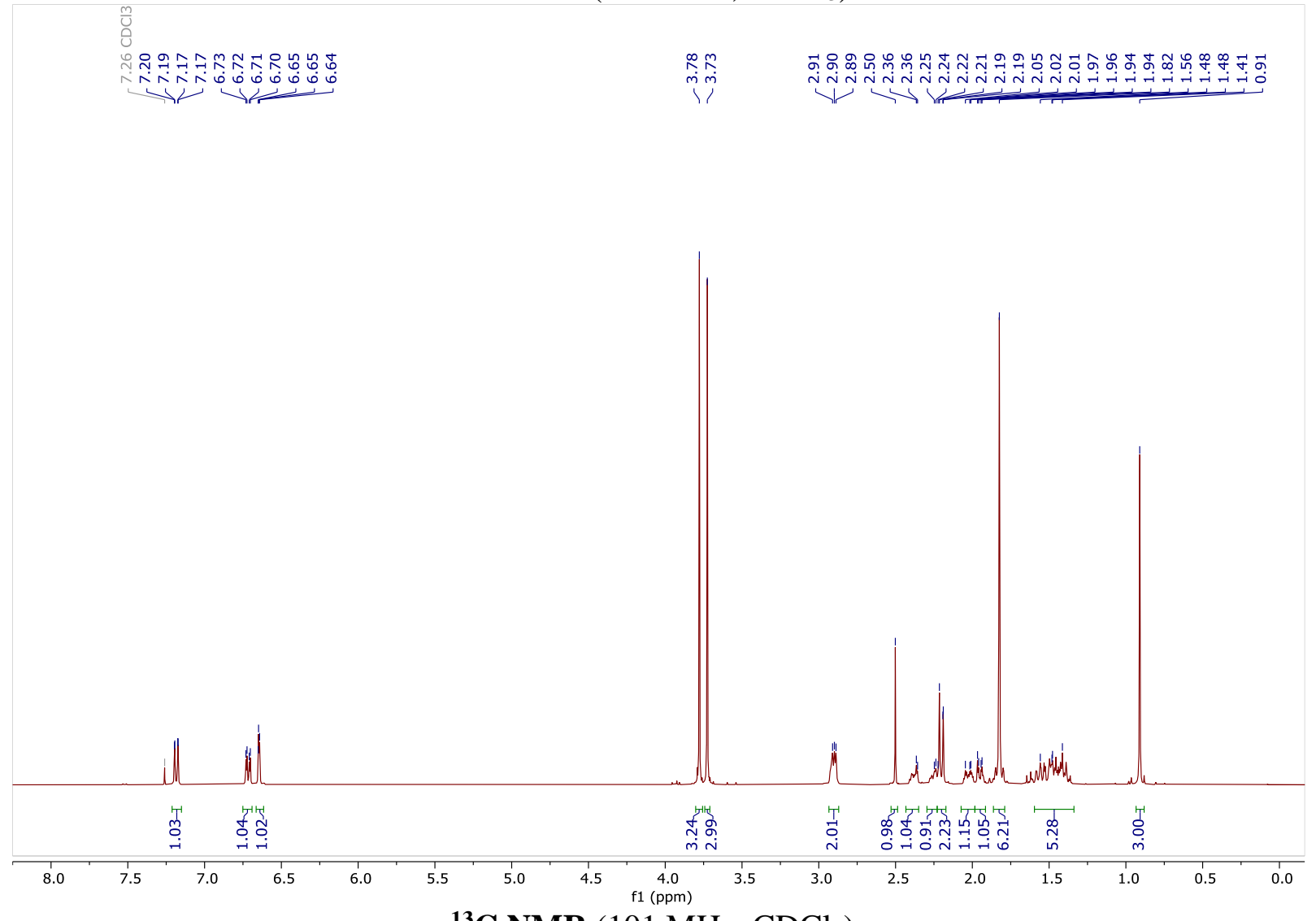

${ }^{13} \mathbf{C ~ N M R}\left(101 \mathrm{MHz}, \mathrm{CDCl}_{3}\right)$

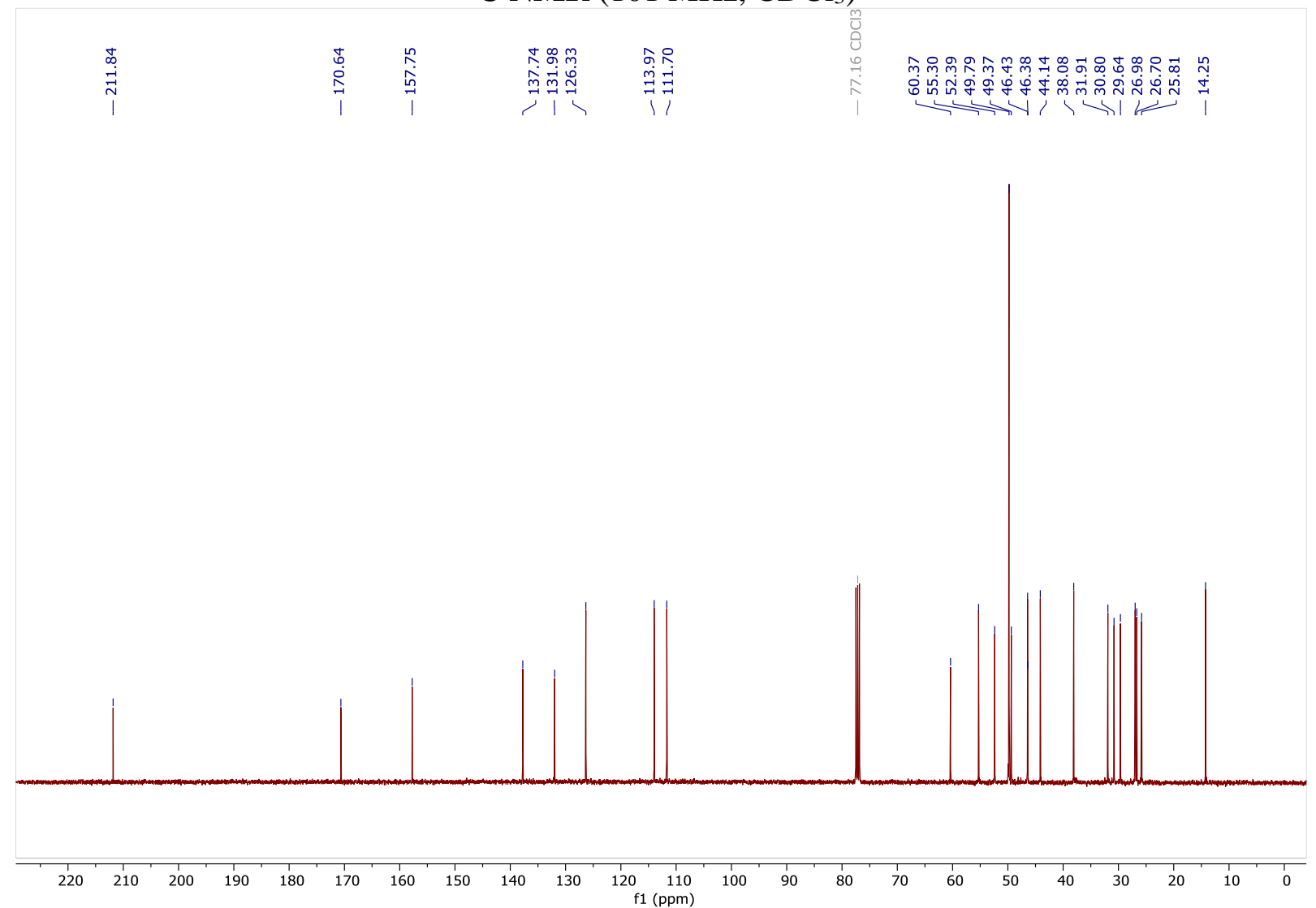


2-(Bicyclo[1.1.1]pentan-1-yl)-6-methoxy-2,3-dihydro-1H-inden-1-one, 4

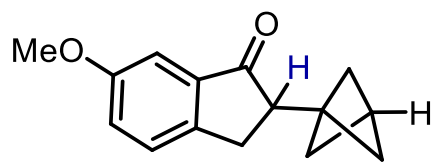

${ }^{\mathbf{1}} \mathbf{H}$ NMR (400 MHz, $\left.\mathrm{CDCl}_{3}\right)$

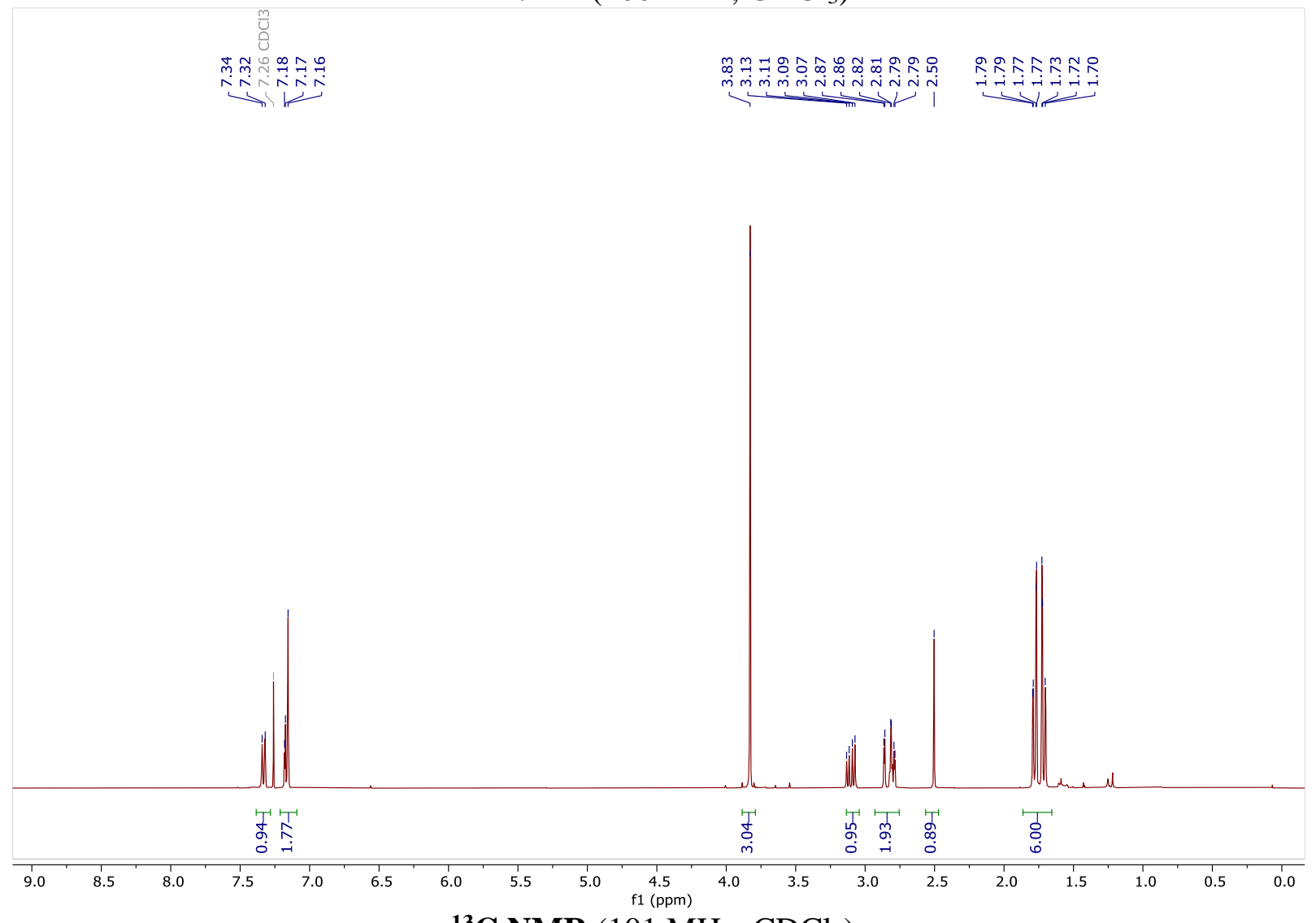

${ }^{13} \mathbf{C}$ NMR $\left(101 \mathrm{MHz}, \mathrm{CDCl}_{3}\right)$

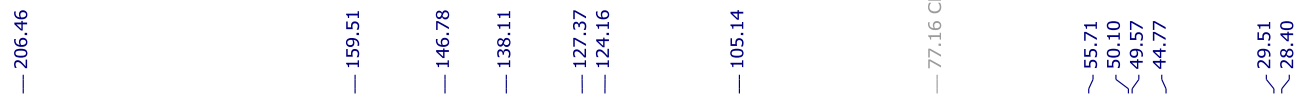

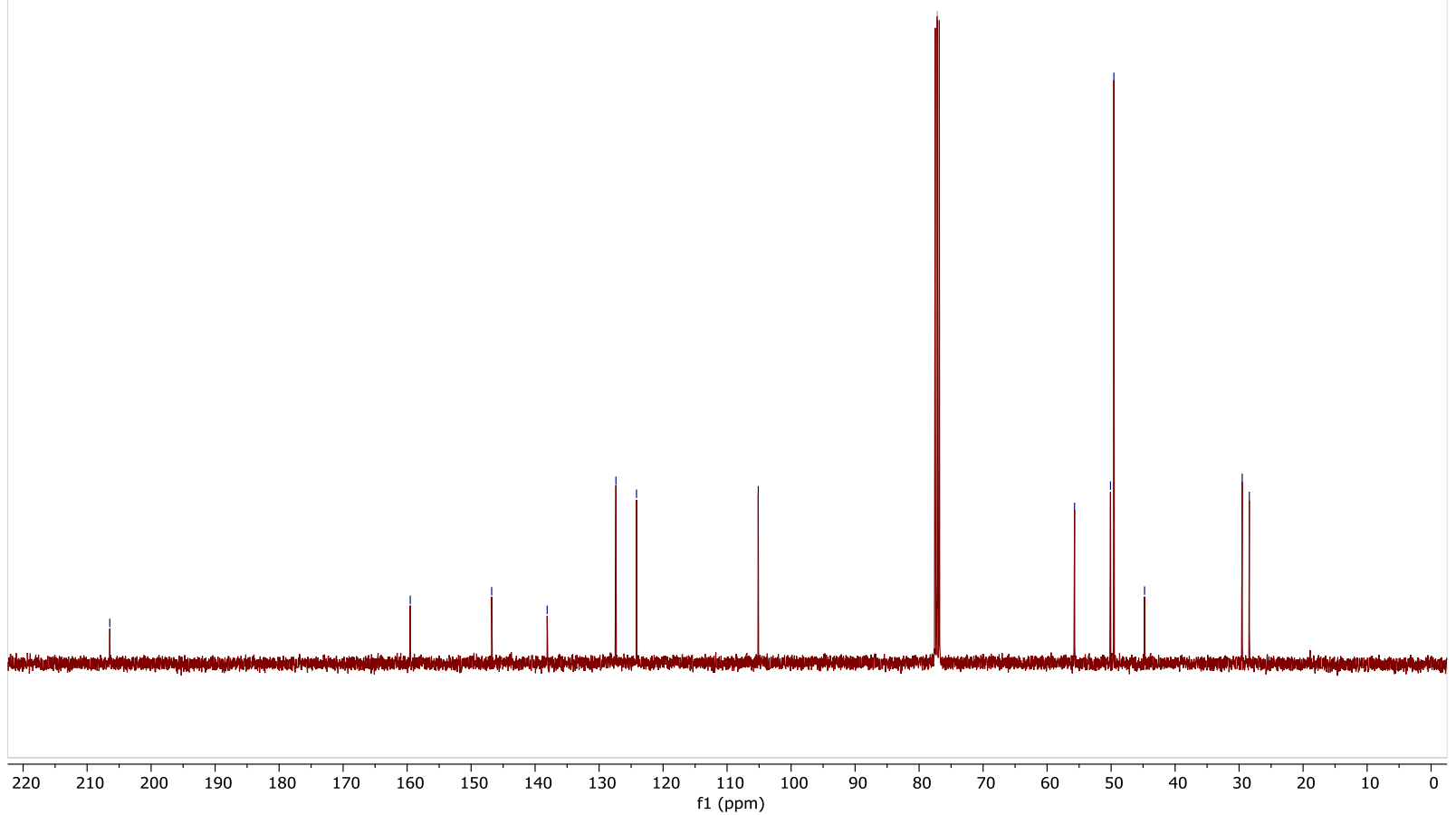


Ethyl 2-(bicyclo[1.1.1]pentan-1-yl)-2-diazoacetate, 5

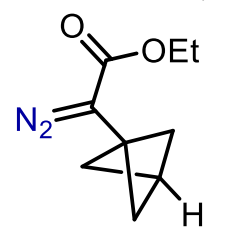

${ }^{1} \mathbf{H}$ NMR $\left(400 \mathrm{MHz}, \mathrm{CDCl}_{3}\right)$

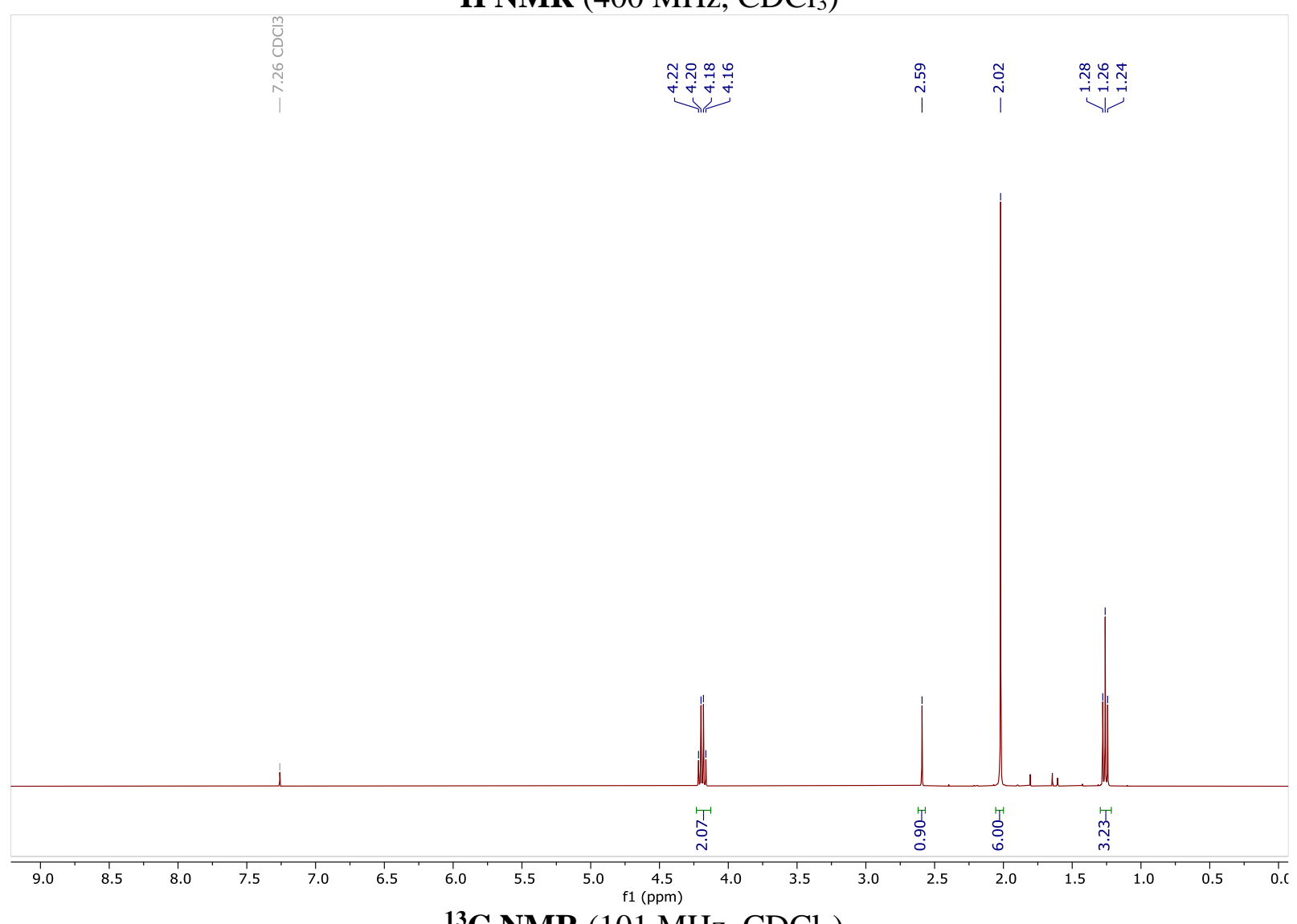

${ }^{13} \mathbf{C}$ NMR (101 MHz, $\left.\mathrm{CDCl}_{3}\right)$

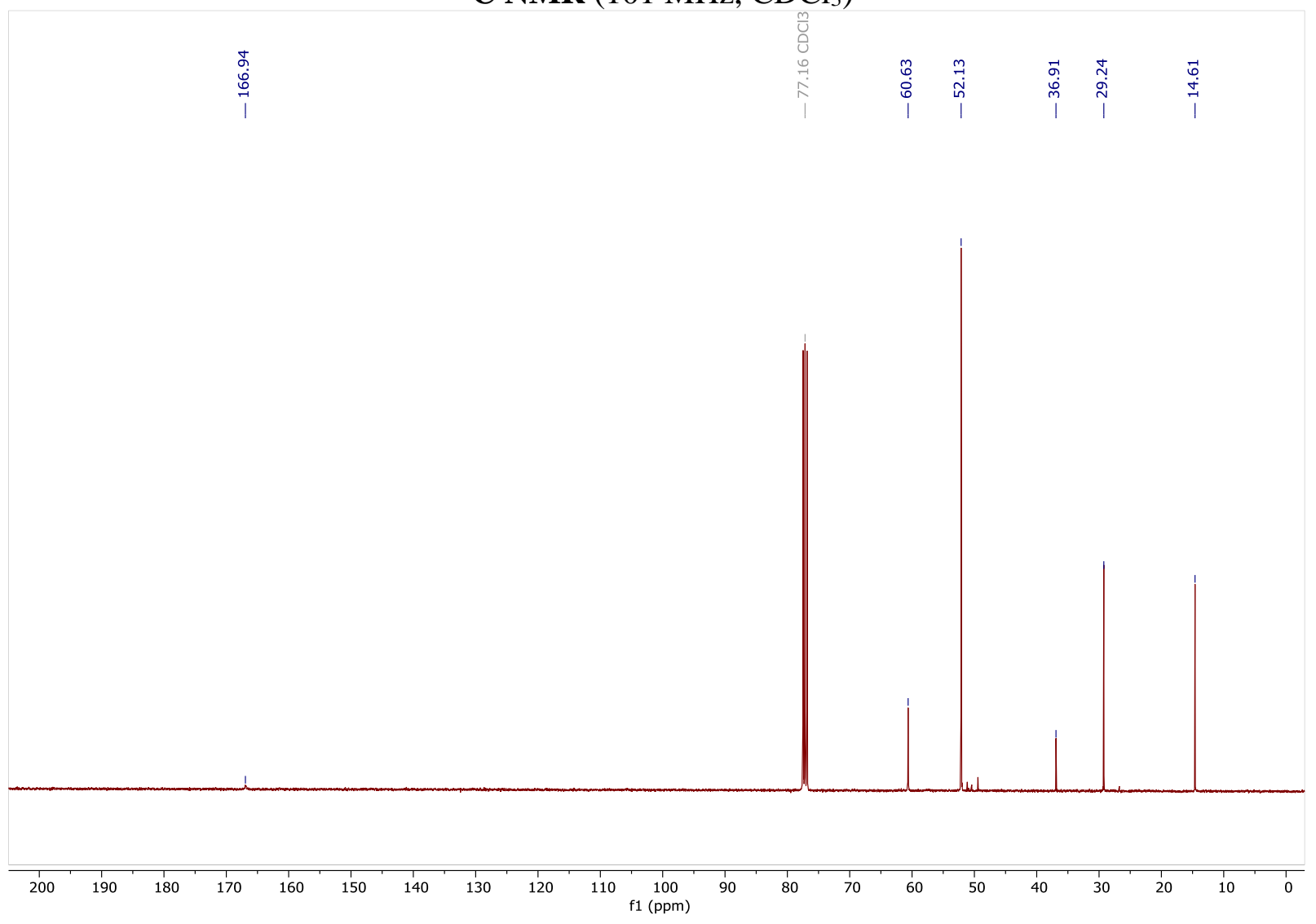

S 80 
1-Benzyl-4-(bicyclo[1.1.1]pentan-1-yl)-2,3-dihydro-1H-pyrrole, 6

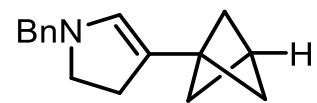

${ }^{1} \mathbf{H}$ NMR $\left(400 \mathrm{MHz}, \mathrm{CDCl}_{3}\right)$

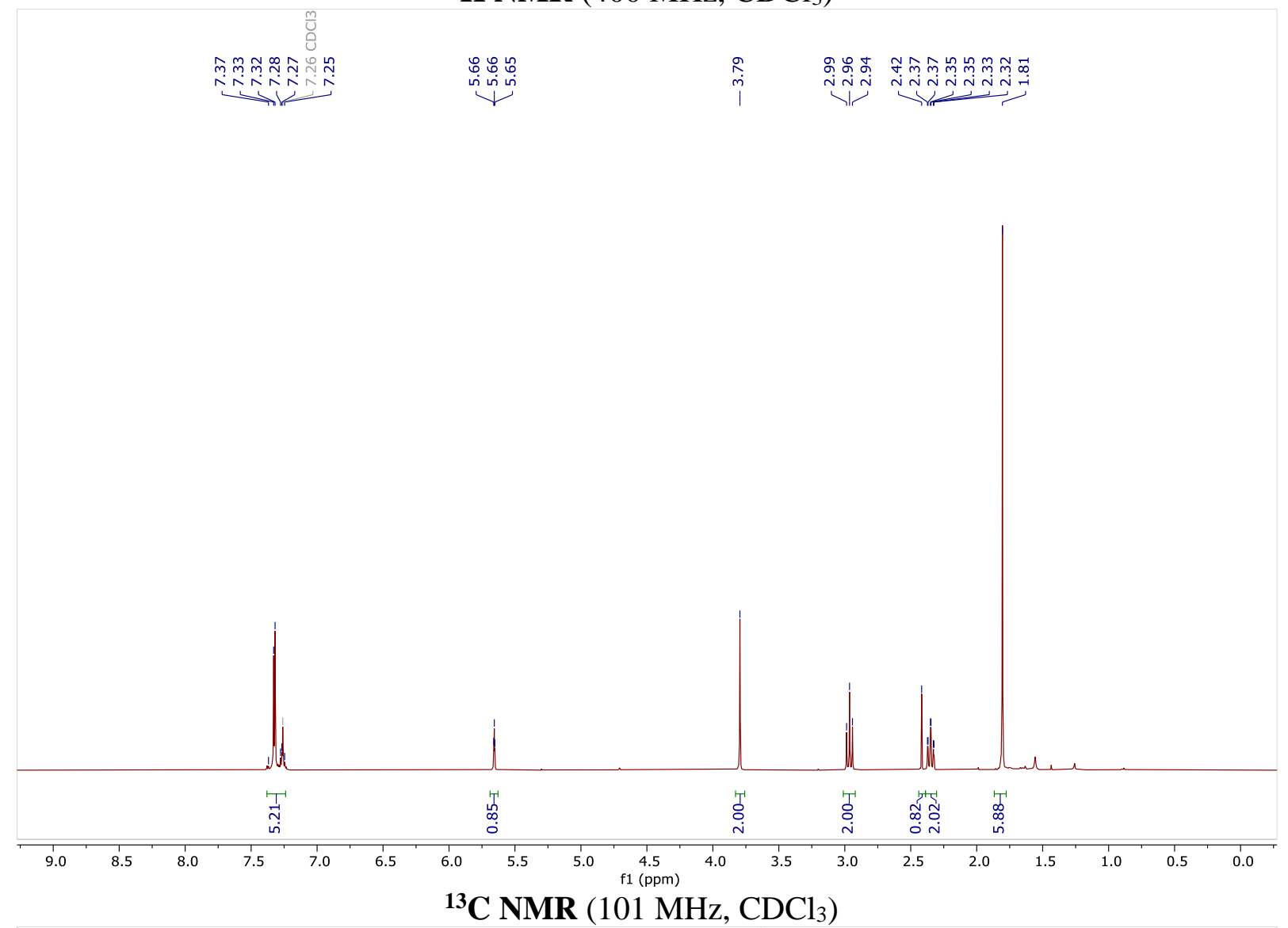

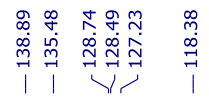

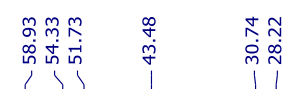

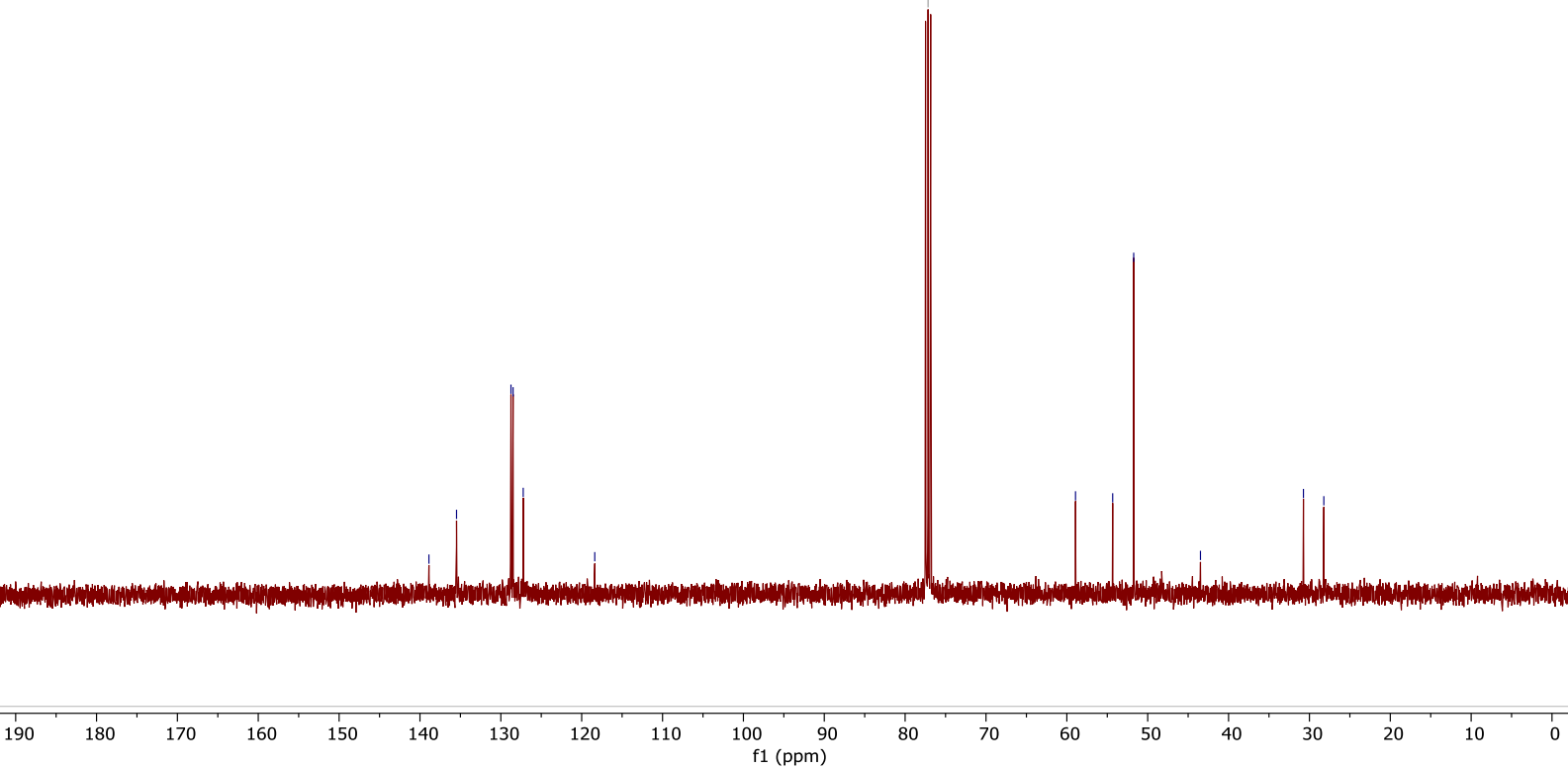


4-(bicyclo[1.1.1]pentan-1-yl)-3,5-diphenyl-1H-pyrazole, 7

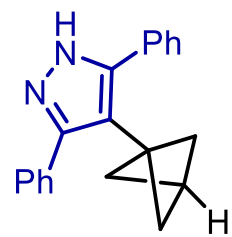

${ }^{1} \mathbf{H}$ NMR $\left(400 \mathrm{MHz}, d_{6}\right.$-DMSO)

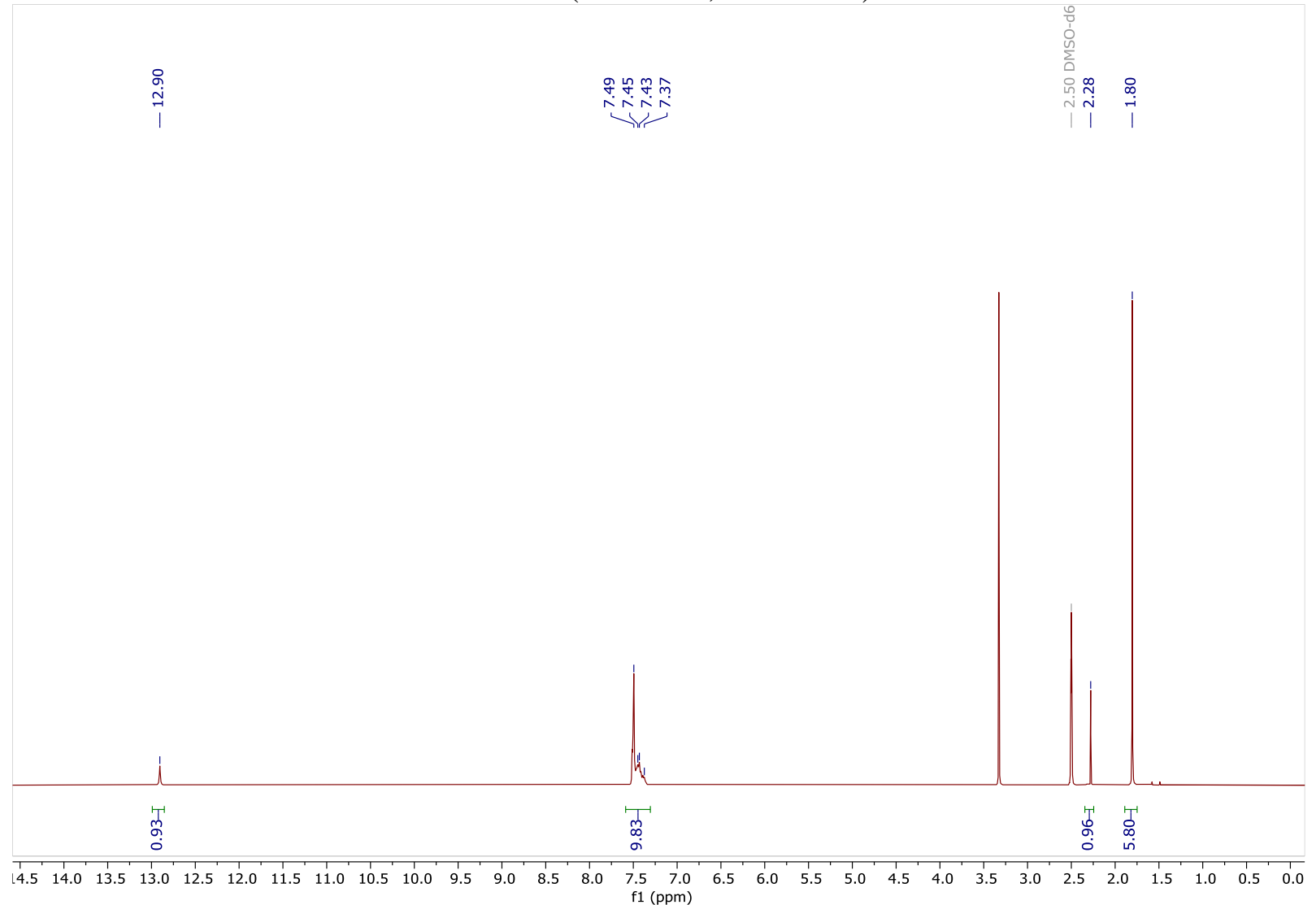

${ }^{13}$ C NMR (101 MHz, $d_{6}$-DMSO)

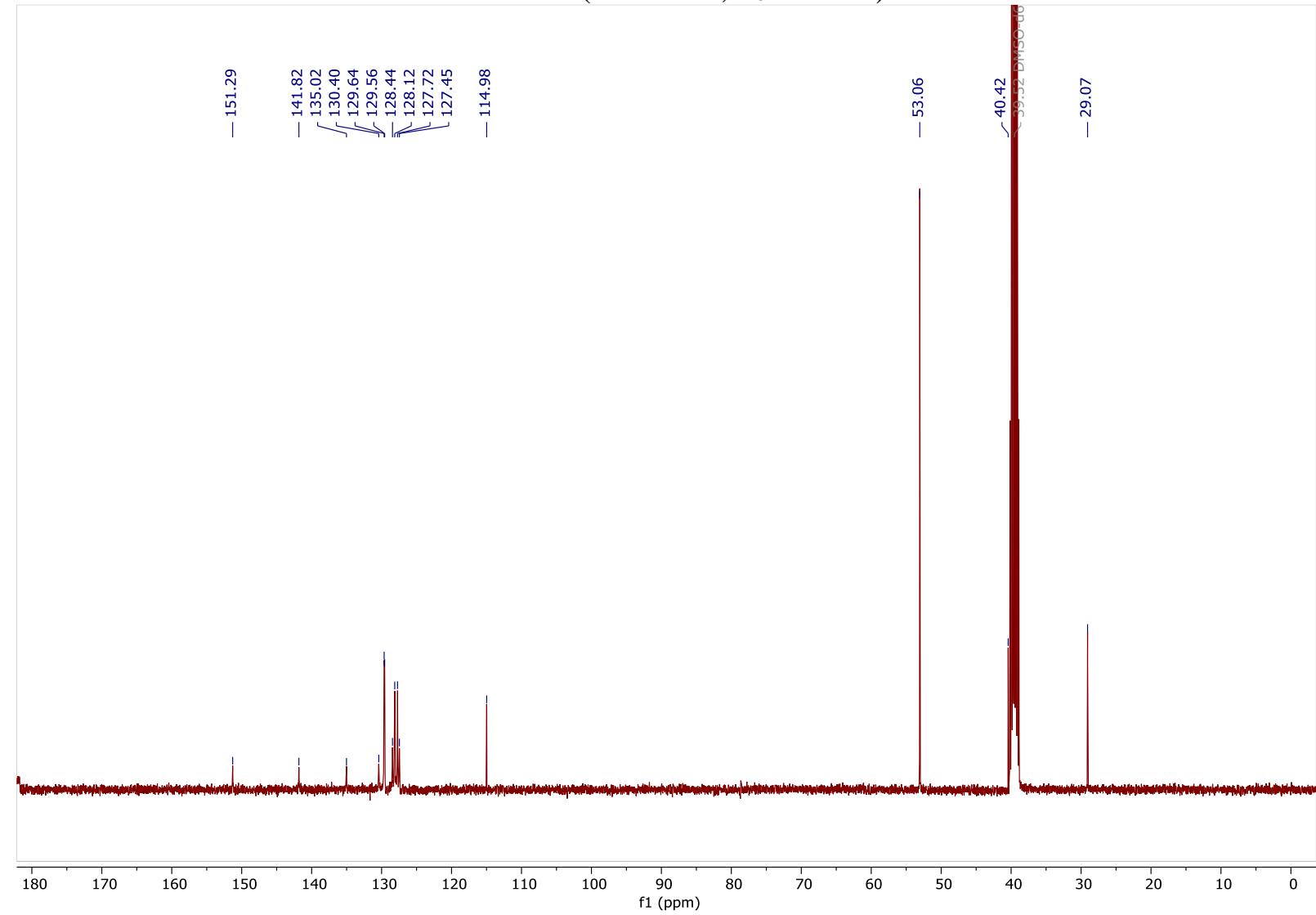


4-(Bicyclo[1.1.1]pentan-1-yl)-3,5-diphenylisoxazole, 8

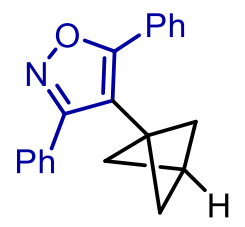

${ }^{\mathbf{1}} \mathbf{H} \mathbf{N M R}\left(400 \mathrm{MHz}, \mathrm{CDCl}_{3}\right)$

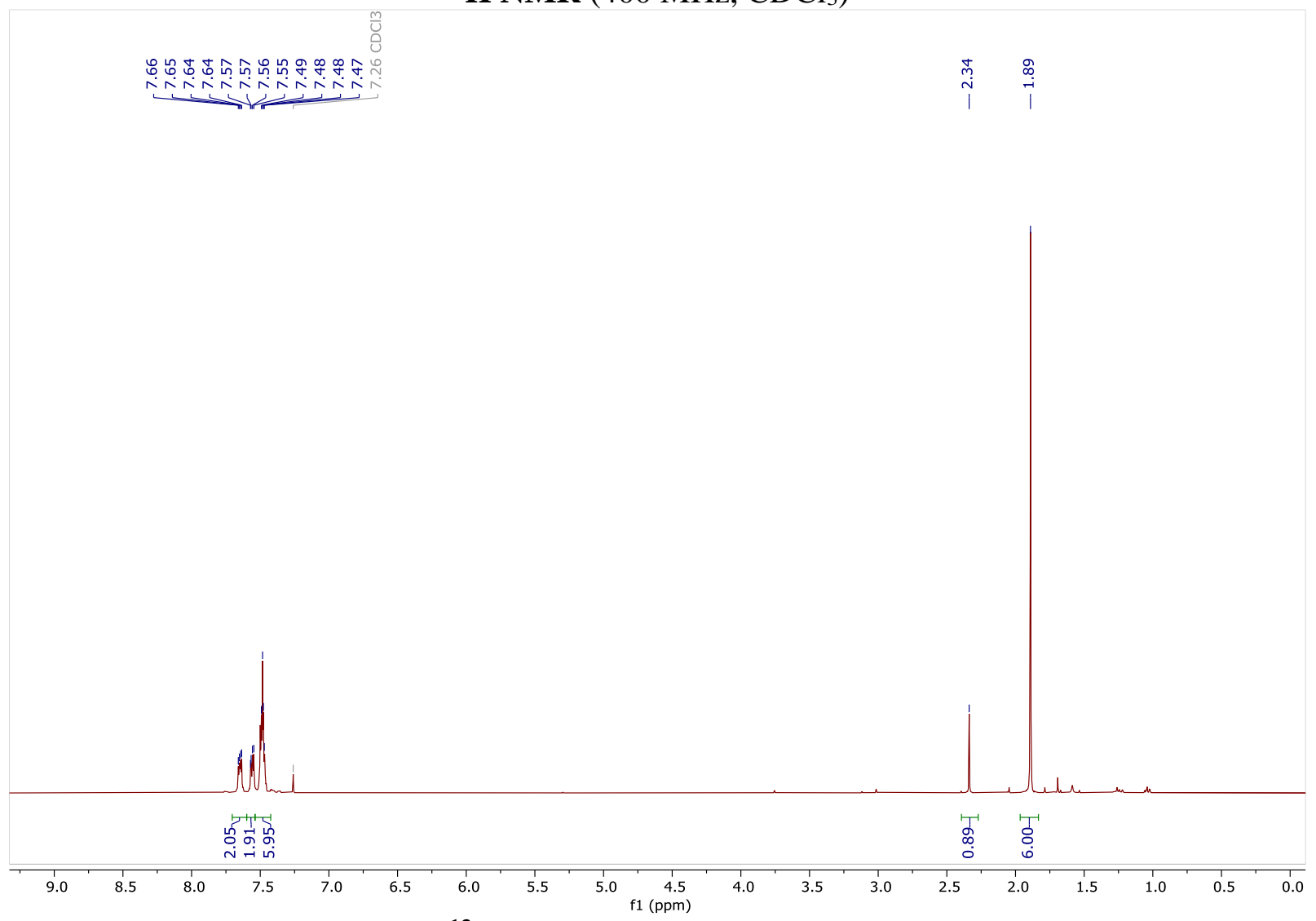

${ }^{13} \mathbf{C}$ NMR (101 MHz, $\left.\mathrm{CDCl}_{3}\right)$

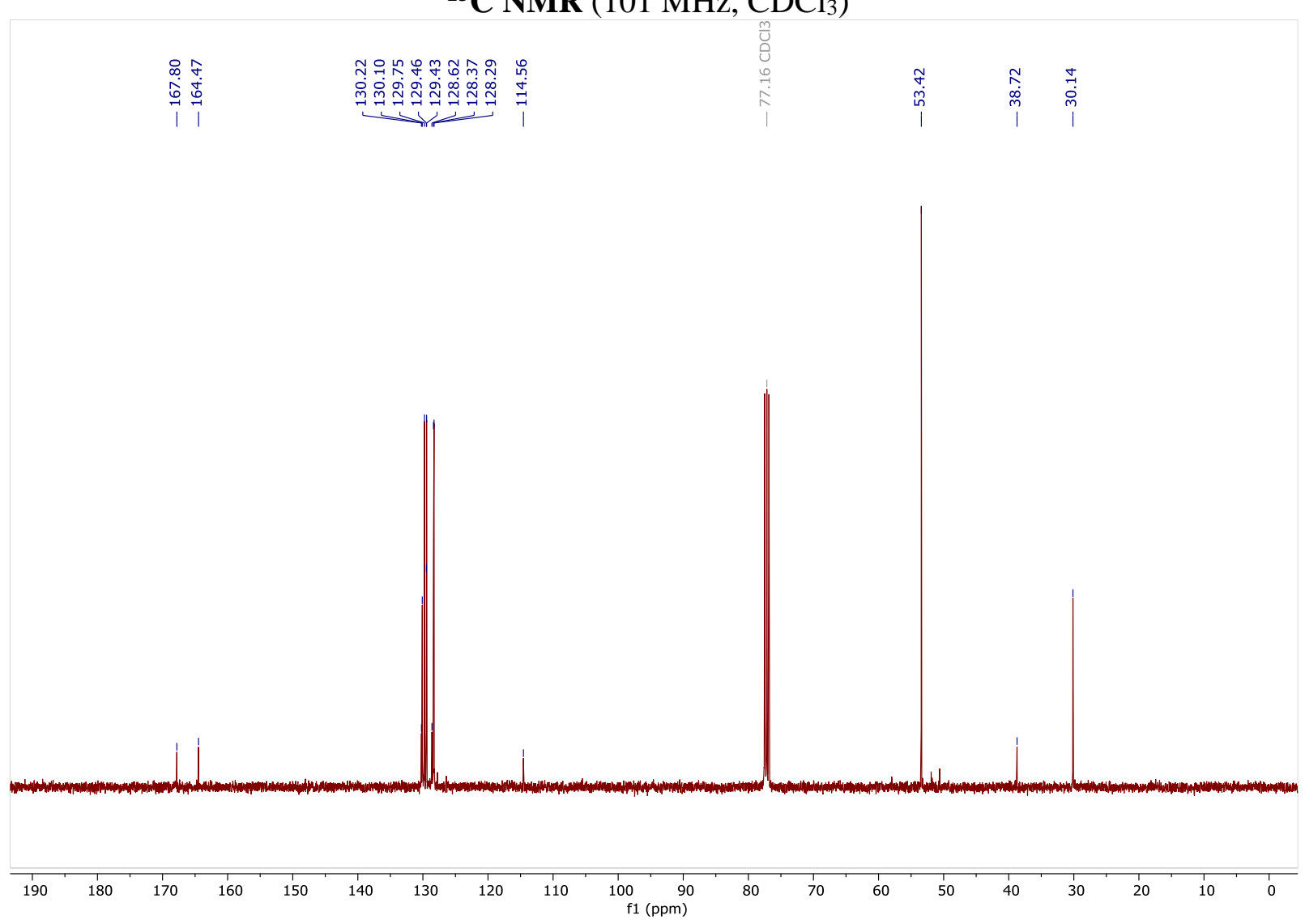

S83 
Benzyl (S)-3-allyl-3-(bicyclo[1.1.1]pentan-1-yl)-4-oxopiperidine-1-carboxylate, 9<smiles>C=CC[C@]12C(=O)CCN(C(=O)O)C1C1CC12</smiles>

${ }^{\mathbf{1}} \mathbf{H} \mathbf{N M R}\left(400 \mathrm{MHz}, \mathrm{CDCl}_{3}\right)$

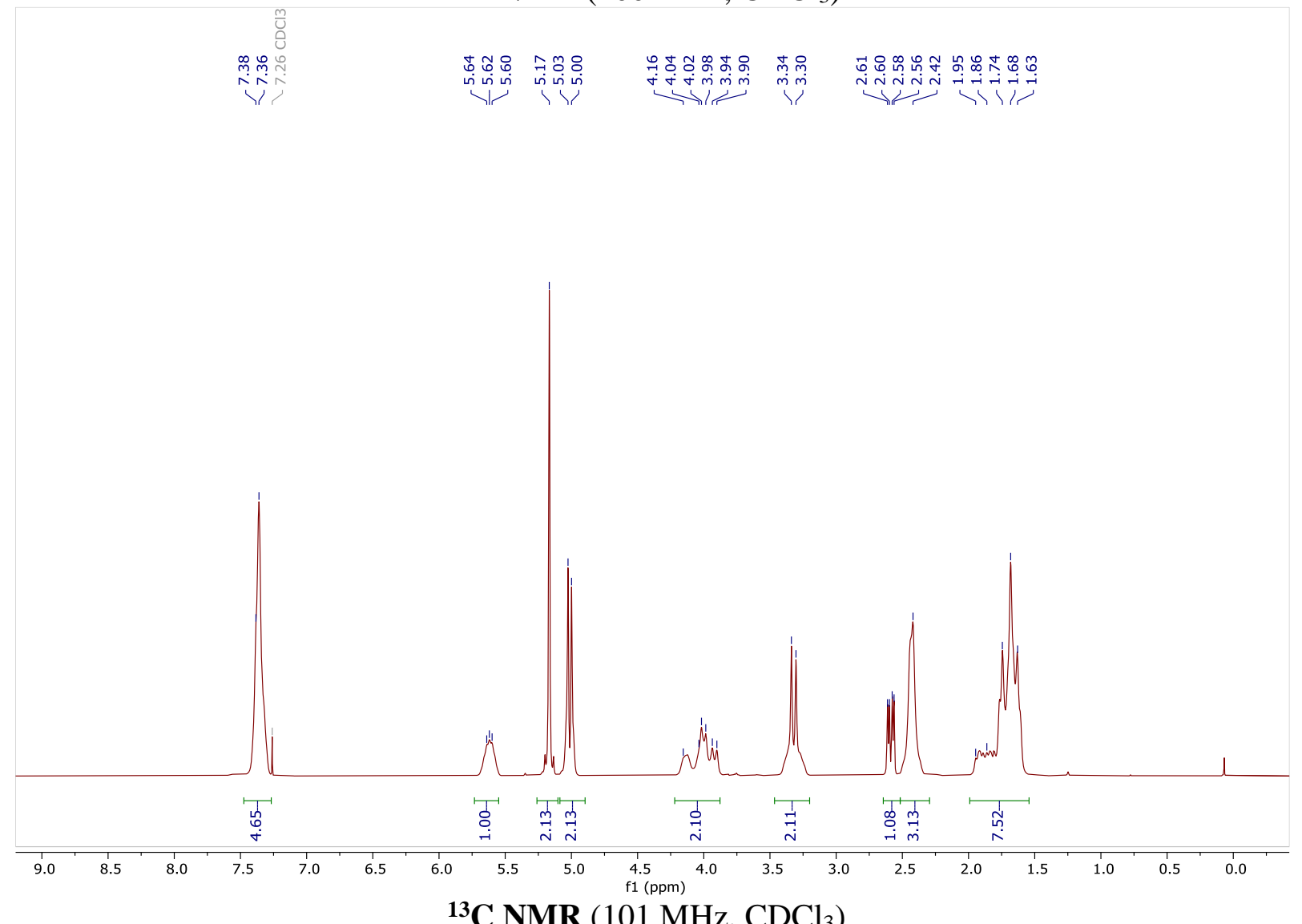

${ }^{13} \mathbf{C ~ N M R}\left(101 \mathrm{MHz}, \mathrm{CDCl}_{3}\right)$

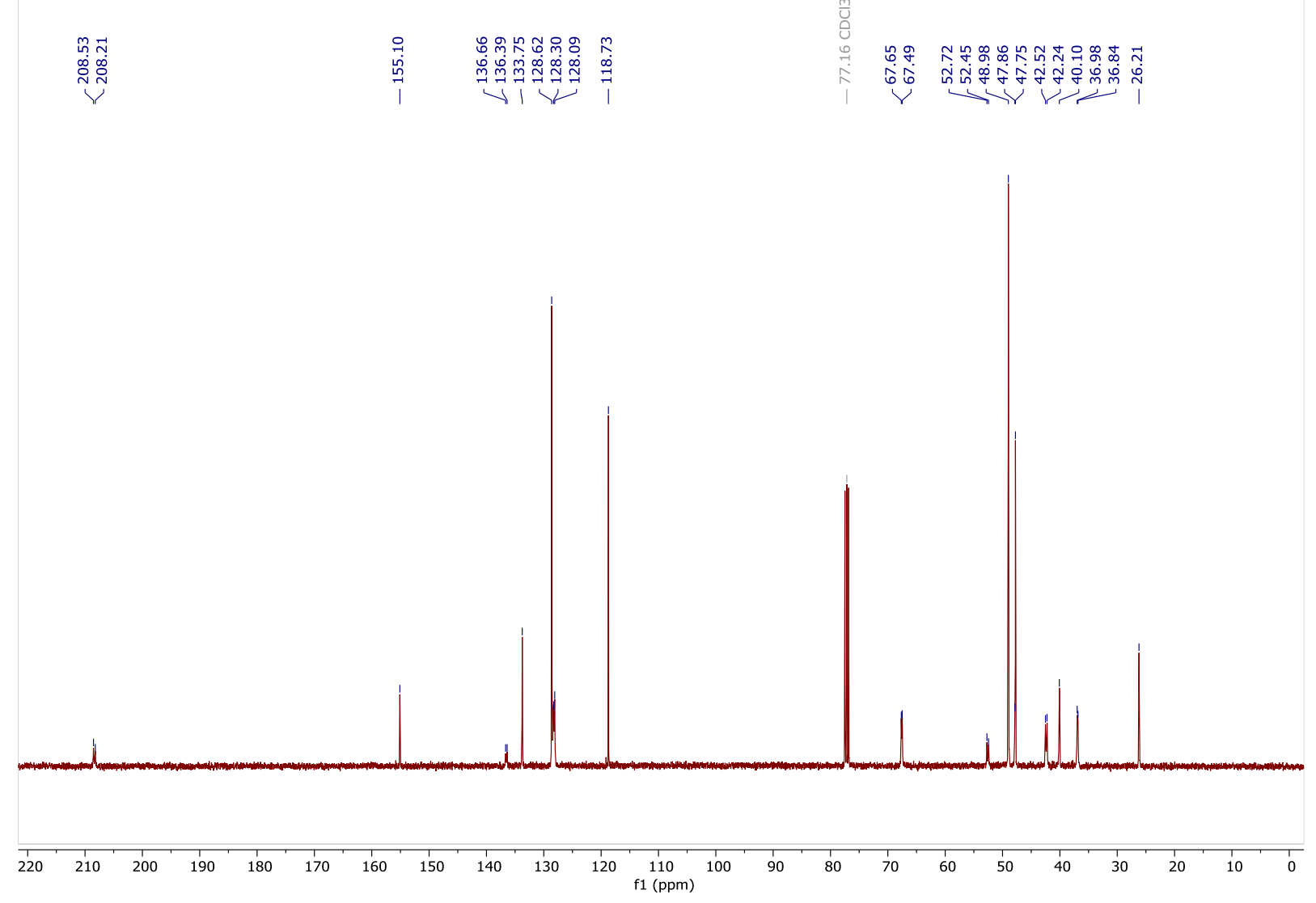


(R)-2-Allyl-2-(bicyclo[1.1.1]pentan-1-yl)-6-methoxy-2,3-dihydro-1H-inden-1-one, 10

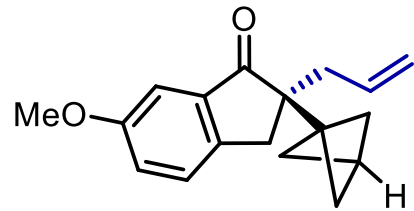

${ }^{1} \mathbf{H}$ NMR $\left(400 \mathrm{MHz}, \mathrm{CDCl}_{3}\right)$

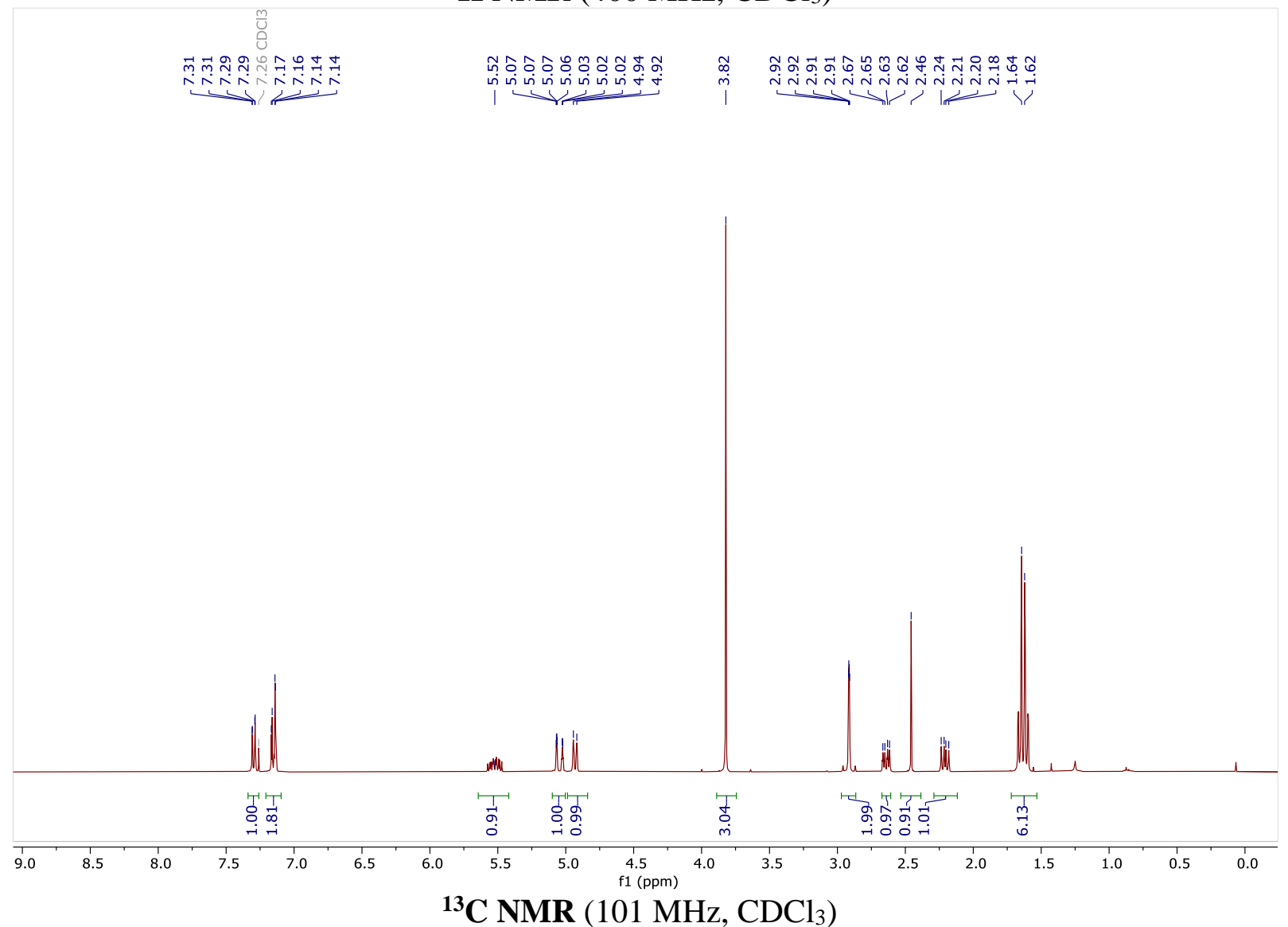

${ }^{13} \mathbf{C}$ NMR $\left(101 \mathrm{MHz}, \mathrm{CDCl}_{3}\right)$

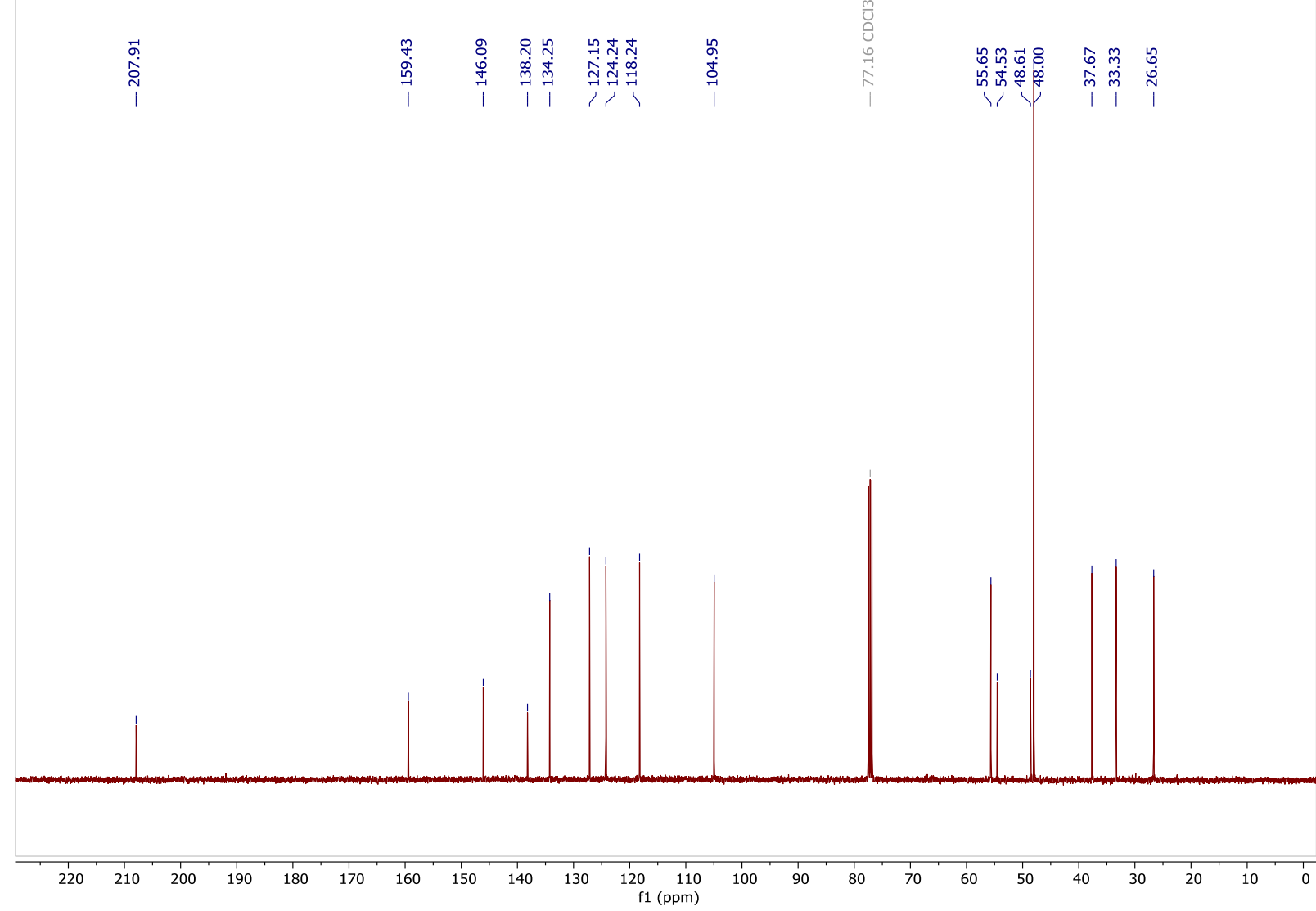


Methyl 7-(((benzyloxy)carbonyl)amino)-3-oxoheptanoate, 2x<smiles>COC(=O)CC(=O)CCCCNC(C)=O</smiles>

${ }^{1} \mathbf{H}$ NMR $\left(400 \mathrm{MHz}, \mathrm{CDCl}_{3}\right)$

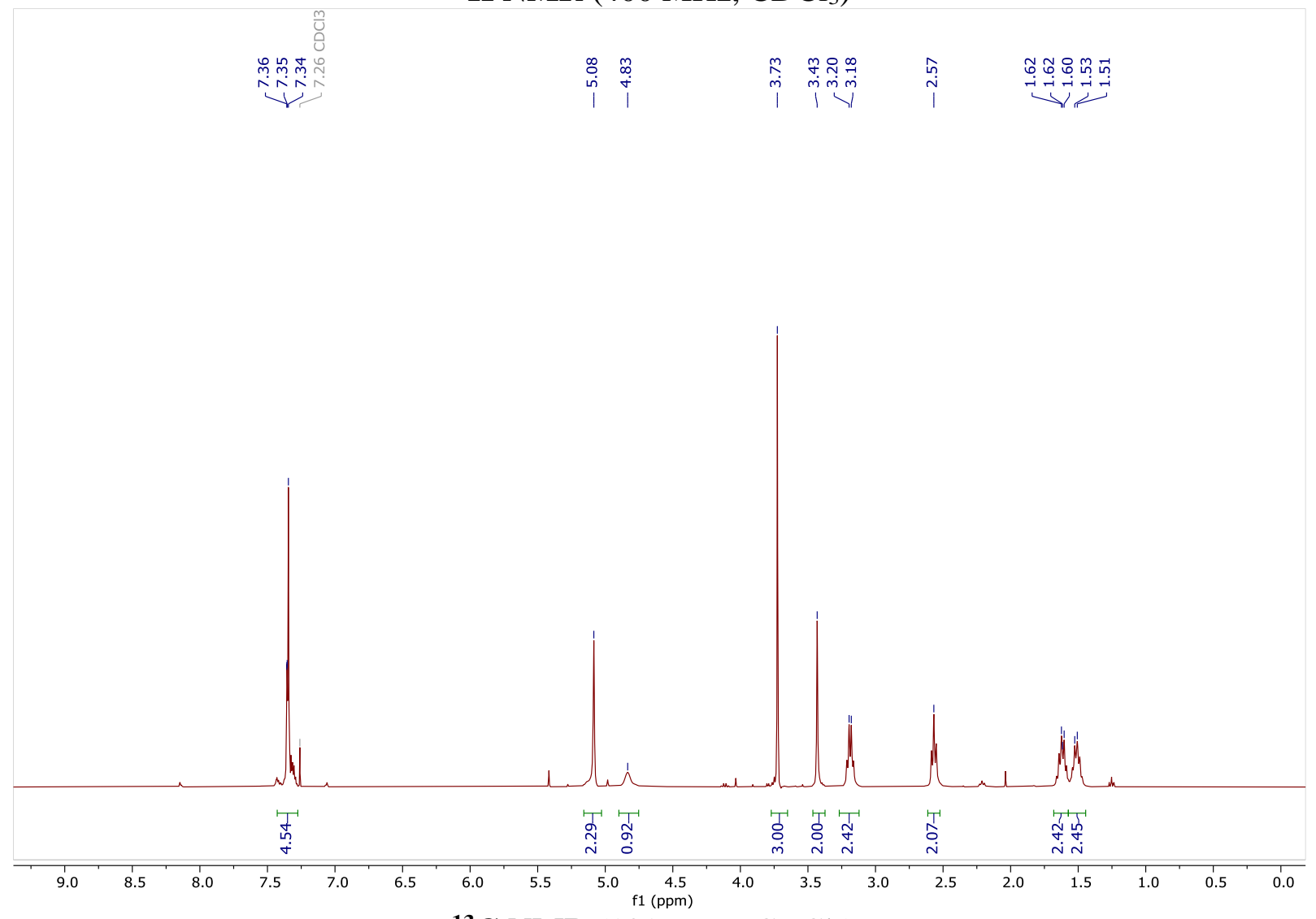

${ }^{13} \mathrm{C}$ NMR (101 MHz, $\left.\mathrm{CDCl}_{3}\right)$

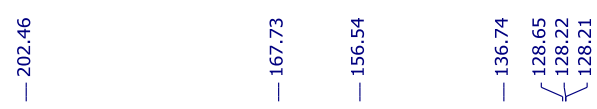

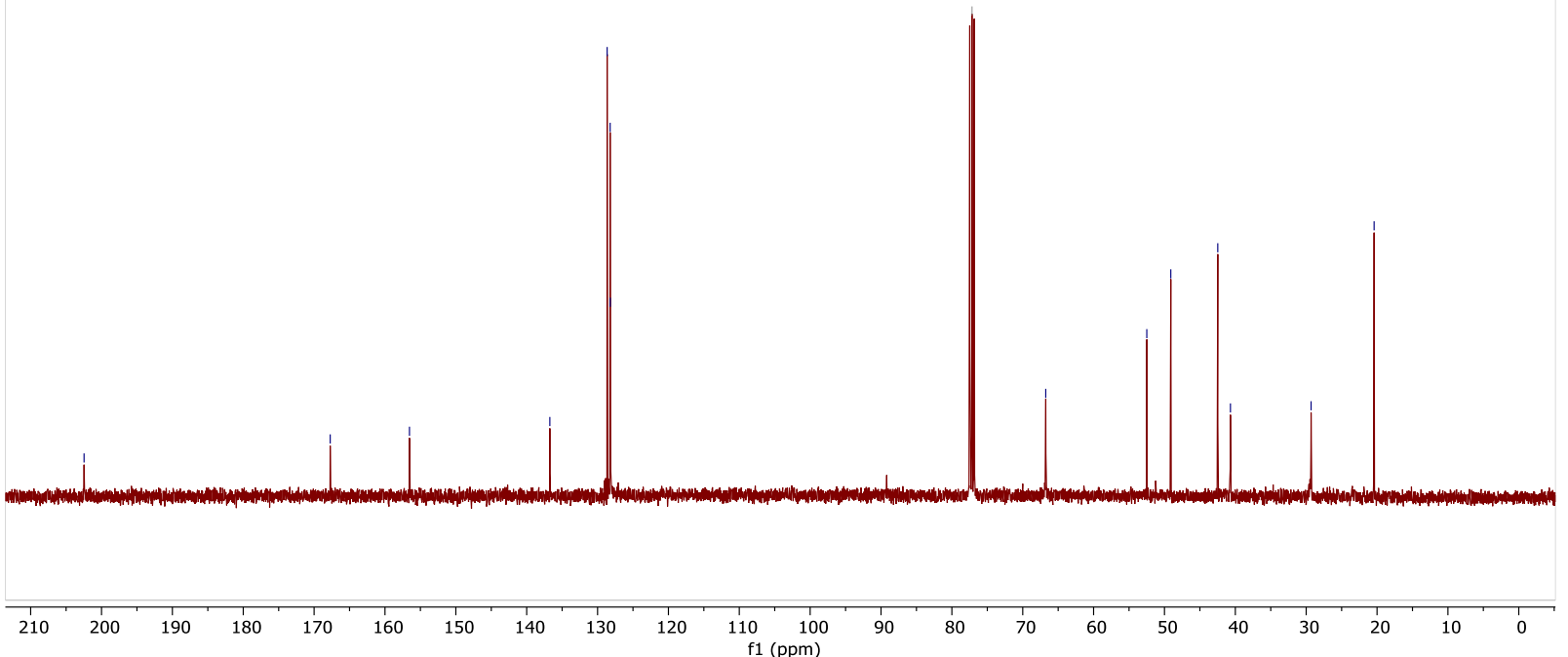


Methyl 7-(((benzyloxy)carbonyl)amino)-2-(bicyclo[1.1.1]pentan-1-yl)-3-oxoheptanoate, 3x

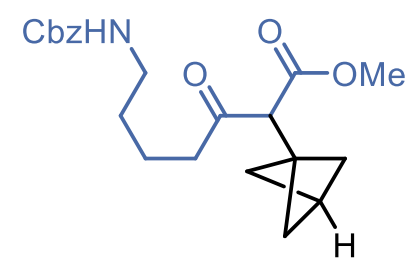

${ }^{1} \mathbf{H}$ NMR (400 MHz, $\left.\mathrm{CDCl}_{3}\right)$

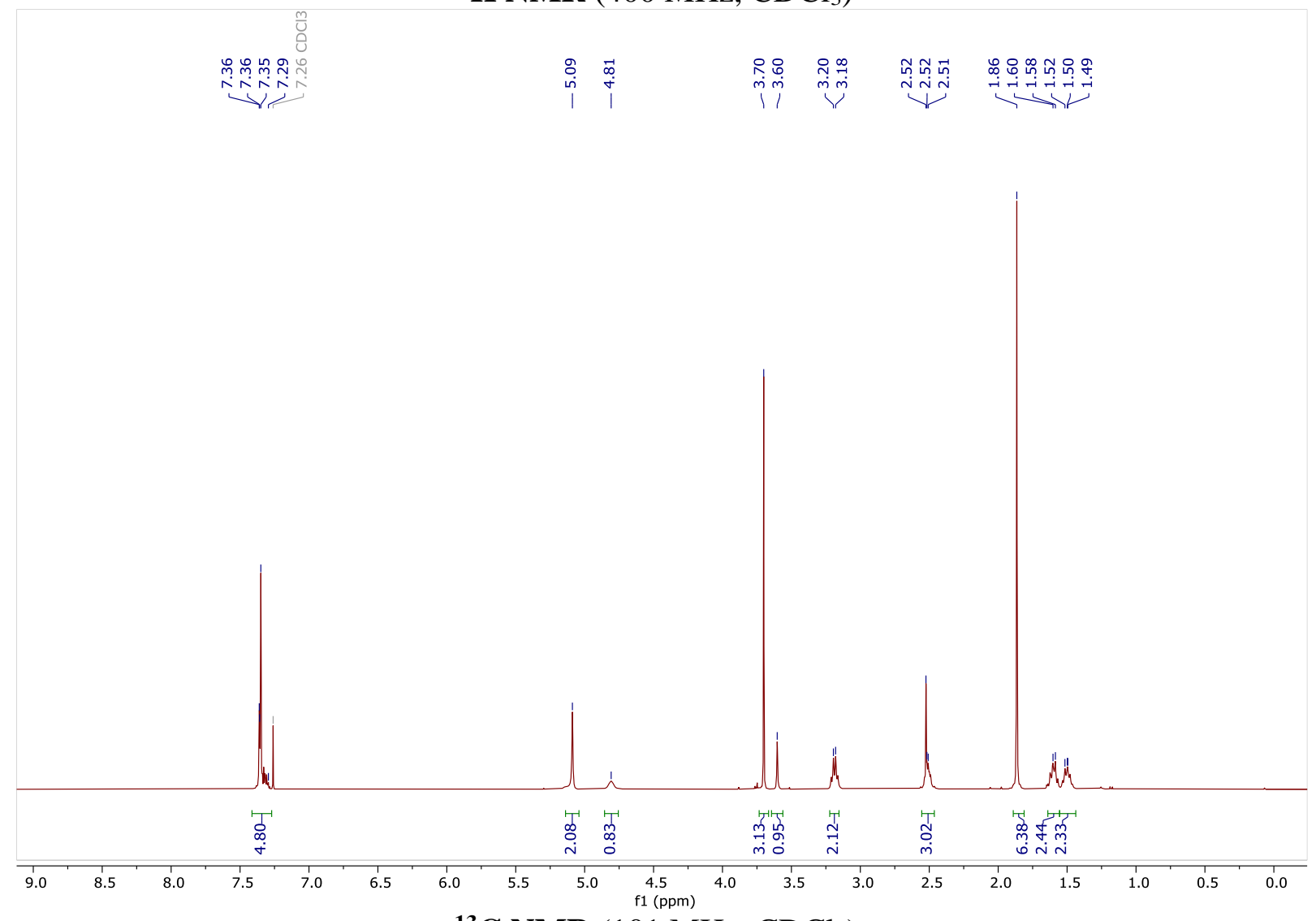

${ }^{13} \mathbf{C}$ NMR (101 MHz, $\left.\mathrm{CDCl}_{3}\right)$

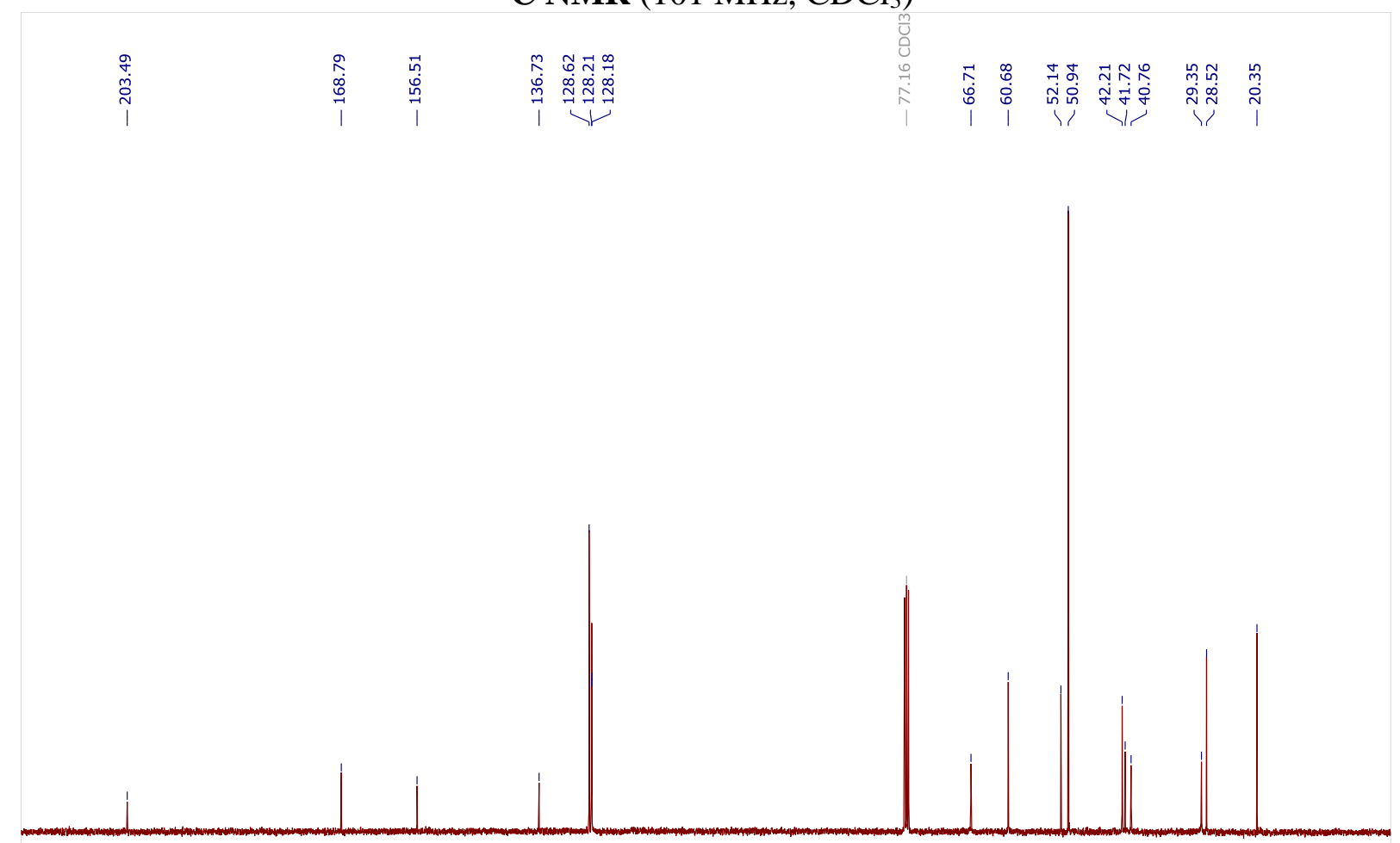

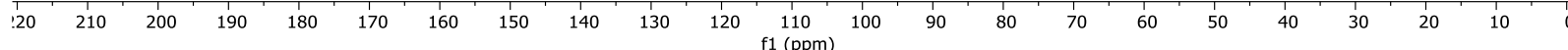




\section{BCP Ritalin, 11}

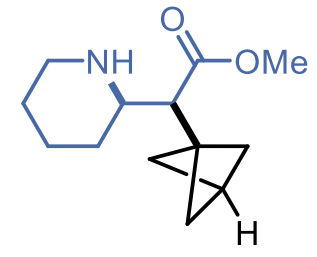

${ }^{1} \mathbf{H}$ NMR $\left(400 \mathrm{MHz}, \mathrm{CDCl}_{3}\right)$

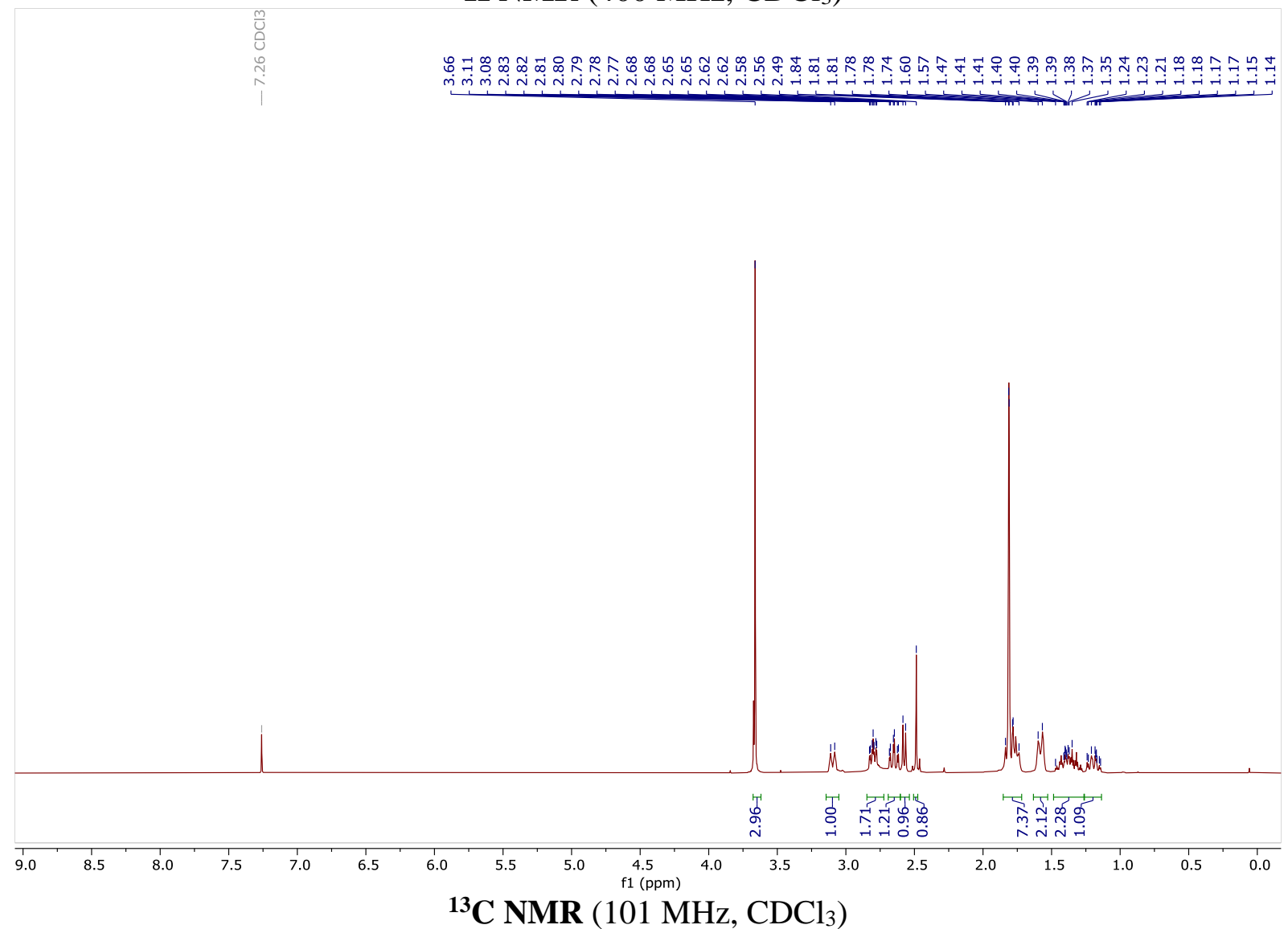

${ }^{13} \mathbf{C}$ NMR $\left(101 \mathrm{MHz}, \mathrm{CDCl}_{3}\right)$

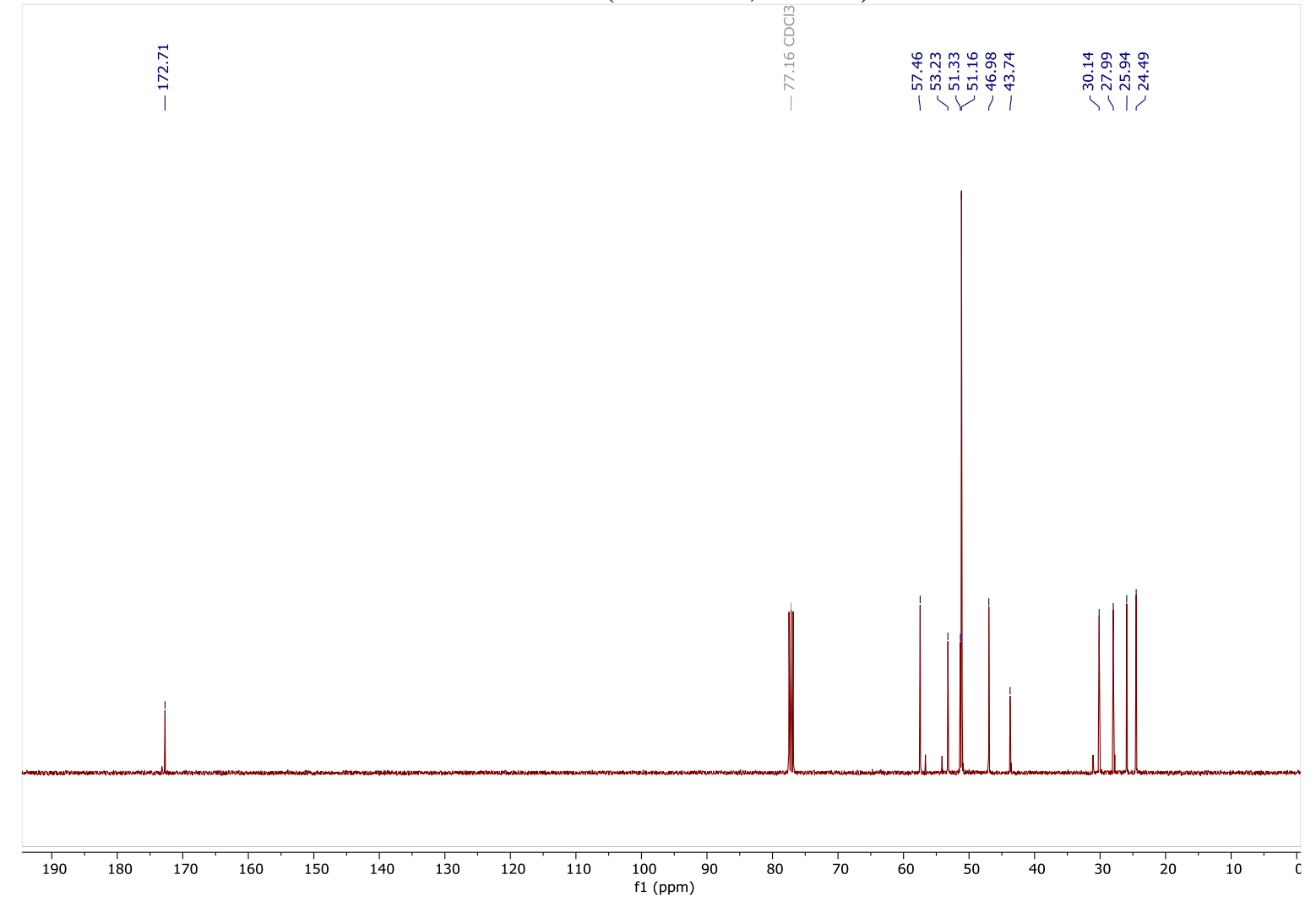


Dimethyl 4-oxopiperidine-1,3-dicarboxylate, $2 \mathrm{~h}$<smiles>CC(=O)C1=C(O)CCN(C(C)=O)C1</smiles>

${ }^{1} \mathbf{H}$ NMR (400 MHz, $\left.\mathrm{CDCl}_{3}\right)$

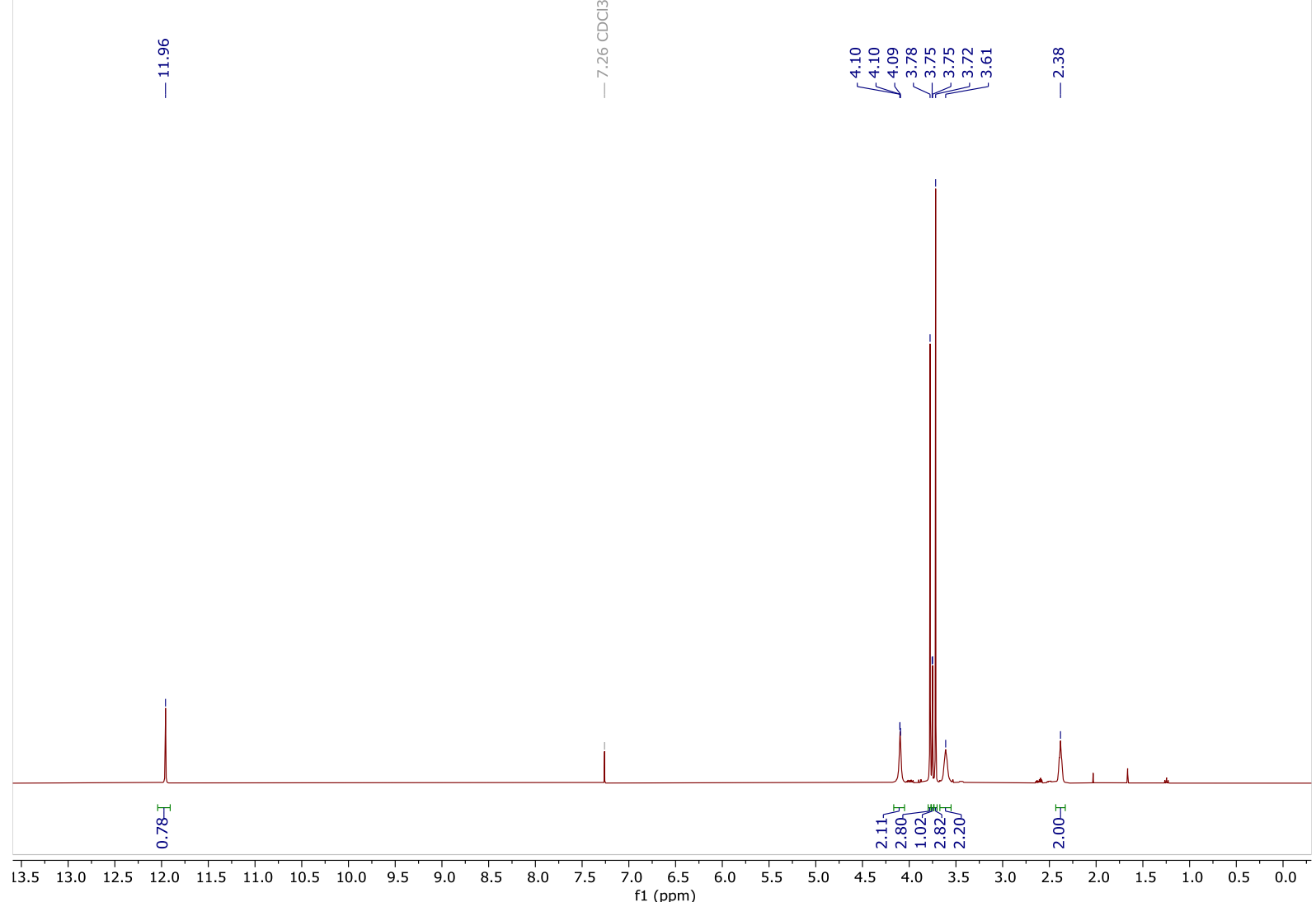


1-Tosylpiperidin-4-one<smiles>CN1CCC(=O)CC1</smiles>

${ }^{1} \mathbf{H}$ NMR $\left(400 \mathrm{MHz}, \mathrm{CDCl}_{3}\right)$

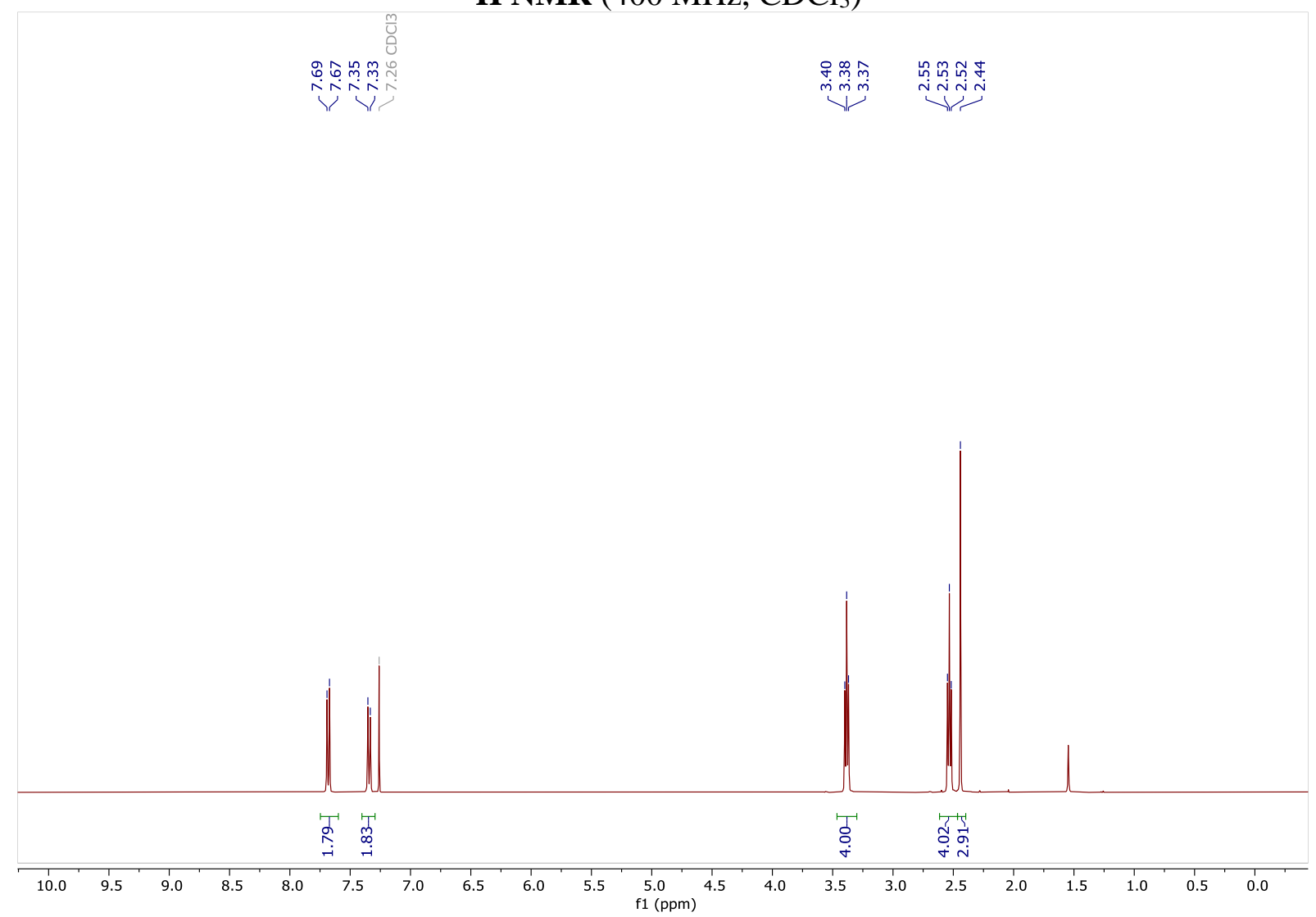


Allyl 4-hydroxy-1-tosyl-1,2,5,6-tetrahydropyridine-3-carboxylate, 2n<smiles>C=CCOC(=O)C1=C(O)CCN(S)C1</smiles>

${ }^{\mathbf{1}} \mathbf{H}$ NMR (400 MHz, $\left.\mathrm{CDCl}_{3}\right)$
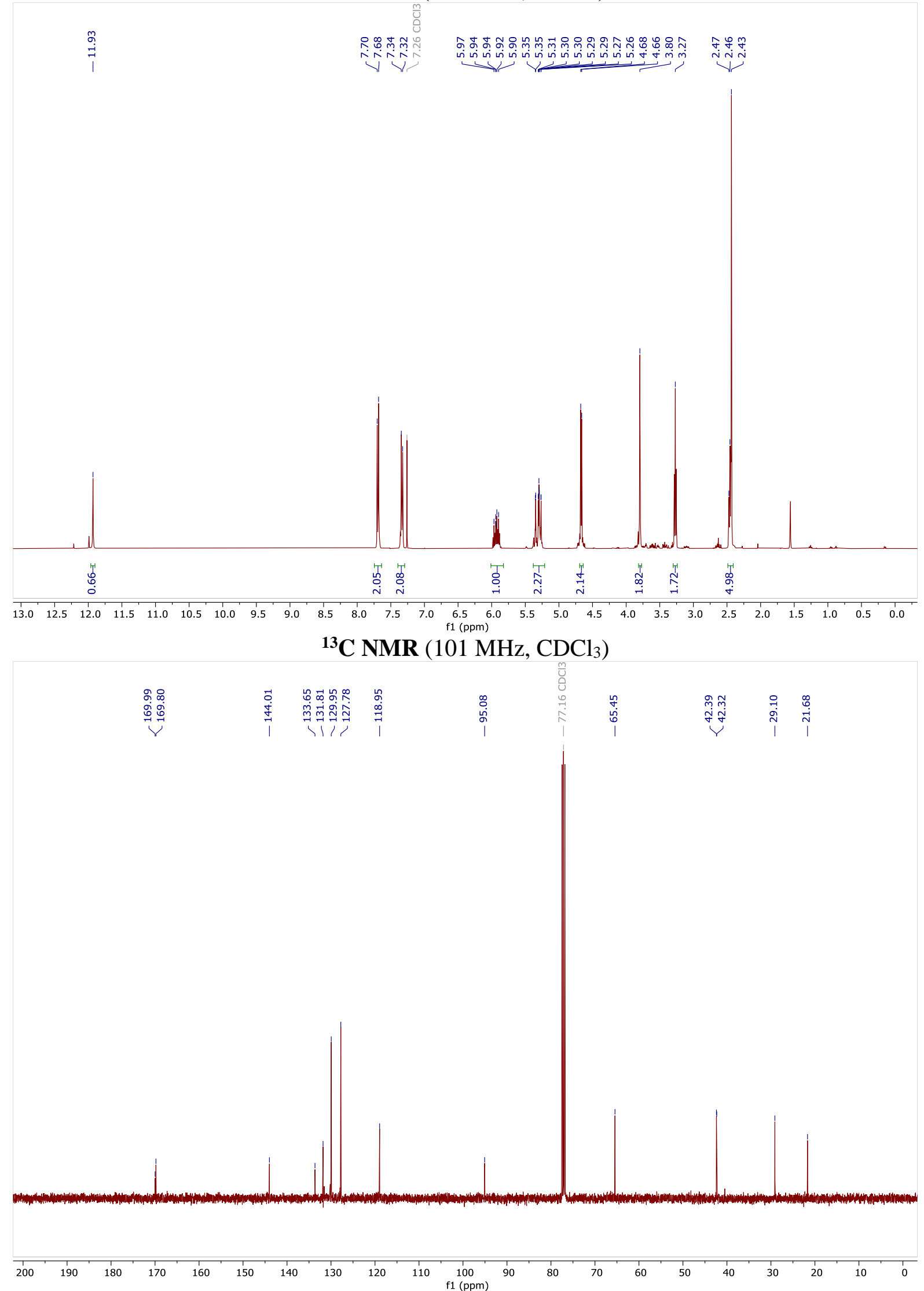


\section{References}

1. $\quad$ E. Speckmeier, T. G. Fischer and K. Zeitler, J. Am. Chem. Soc., 2018, 140, 1535315365.

2. R. Knorr, C. Pires, C. Behringer, T. Menke, J. Freudenreich, E. C. Rossmann and P. Böhrer, J. Am. Chem. Soc., 2006, 128, 14845-14853.

3. M. L. J. Wong, A. J. Sterling, J. J. Mousseau, F. Duarte and E. A. Anderson, Nature Communications, 2021, 12, 1644.

4. D. C. Behenna, J. T. Mohr, N. H. Sherden, S. C. Marinescu, A. M. Harned, K. Tani, M. Seto, S. Ma, Z. Novák, M. R. Krout, R. M. McFadden, J. L. Roizen, J. A. Enquist Jr., D. E. White, S. R. Levine, K. V. Petrova, A. Iwashita, S. C. Virgil and B. M. Stoltz, Chem. Eur. J., 2011, 17, 14199-14223.

5. T. A. Crabb, S. T. Ingate and T. G. Nevell, Magn. Reson. Chem., 1992, 30, 129-132.

6. C. Ezzili, M. Mileni, N. McGlinchey, J. Z. Long, S. G. Kinsey, D. G. Hochstatter, R. C. Stevens, A. H. Lichtman, B. F. Cravatt, E. J. Bilsky and D. L. Boger, J. Med. Chem., 2011, 54, 2805-2822.

7. K. Matsuzaki, K. Okuyama, E. Tokunaga, M. Shiro and N. Shibata, ChemistryOpen, 2014, 3, 233-237.

8. J. Mayol-Llinàs, W. Farnaby and A. Nelson, Chem. Commun., 2017, 53, 12345-12348.

9. R. Šebesta, M. G. Pizzuti, A. J. Boersma, A. J. Minnaard and B. L. Feringa, Chem. Commun., 2005, DOI: 10.1039/B417727D, 1711-1713.

10. M. Dal Prà, D. Carta, G. Szabadkai, M. Suman, Y. Frión-Herrera, N. Paccagnella, G. Castellani, S. De Martin and M. G. Ferlin, Biorg. Med. Chem., 2018, 26, 1686-1704. 